THE ASTROPHYSiCAL Journal SuPPLEMENT SeRIES, 89:123-153, 1993 November

(c) 1993. The American Astronomical Society. All rights reserved. Printed in U.S.A.

\title{
FORMALDEHYDE AS A PROBE OF PHYSICAL CONDITIONS IN DENSE MOLECULAR CLOUDS
}

\author{
JEFFREY G. MANGUM \\ University of Arizona, Steward Observatory; Submillimeter Telescope Observatory, Tucson, AZ 85721; Department of Astronomy, \\ The University of Texas, Austin, TX 78712; and National Radio Astronomy Observatory ${ }^{1}$
}

AND

ALWYN WOOTTEN

National Radio Astronomy Observatory, Edgemont Road, Charlottesville, VA 22903

Received 1992 September 1; accepted 1993 March 9

\begin{abstract}
We present a detailed analysis describing the utility of the formaldehyde $\left(\mathrm{H}_{2} \mathrm{CO}\right)$ molecule in the derivation of the kinetic temperature and spatial density within molecular clouds. Measurements of 13 transitions from both the ortho and para species of $\mathrm{H}_{2} \mathrm{CO}$ have been made toward a sample of 11 active star formation regions. These $\mathrm{H}_{2} \mathrm{CO}$ transitions range in frequency from 211 to $365 \mathrm{GHz}$ and in upper-state energy from 21 to $241 \mathrm{~K}$. This range in excitation has allowed us to analyze $\mathrm{H}_{2} \mathrm{CO}$ sensitivity to both cool $\left(T_{K} \lesssim 50 \mathrm{~K}\right)$ and warm $\left(T_{K}>50 \mathrm{~K}\right)$ molecular material.

Using a spherical large velocity gradient (LVG) model to solve for the excitation of $\mathrm{H}_{2} \mathrm{CO}$, we analyze the sensitivity of several ortho- and para- $\mathrm{H}_{2} \mathrm{CO}$ transition intensity ratios to the kinetic temperature and spatial density within molecular clouds. Through this analysis we derive several "rules of thumb" which should be followed when measurements of a particular intensity ratio are used to calculate $T_{K}$ or $n\left(\mathrm{H}_{2}\right)$ in a molecular cloud. We find that for $T_{K} \lesssim 150 \mathrm{~K}$ and over ranges in $\mathrm{H}_{2} \mathrm{CO}$ column density typical for most molecular clouds, several $\mathrm{H}_{2} \mathrm{CO}$ transition intensity ratios are excellent monitors of $T_{K}$ and $n\left(\mathrm{H}_{2}\right)$. Since the transitions whose relative intensities are sensitive to kinetic temperature can be measured using the same receiving system (and can in some cases be measured within the same spectrum), calibration uncertainties are minimized. We also present a detailed analysis of the uncertainties encountered in our modeling procedure, including the potential importance of infrared excitation.

Using our measured $\mathrm{H}_{2} \mathrm{CO}$ radiation temperatures, we have constrained LVG model solutions for the kinetic temperature, spatial density, and $\mathrm{H}_{2} \mathrm{CO}$ species column density in each of the sources in our sample. Our derived spatial densities are comparable to those estimated using other molecular tracers. In all of the regions in our sample, though, we measure kinetic temperatures greater than $50 \mathrm{~K}$, significantly higher than previous estimates for many of these sources. Previous underestimation of $T_{K}$ is due to the use of tracers which are sensitive only to cool $\left(T_{K} \lesssim 50 \mathrm{~K}\right)$ gas. In particular, temperatures of $100 \mathrm{~K}$ or more occur both toward the cool young binary object IRAS 16293-2422 and toward the "protostellar condensations" FIR 4/FIR 5 in NGC 2024.

Using our ortho- and para- $\mathrm{H}_{2} \mathrm{CO}$ measurements we have calculated the $N\left(\right.$ ortho- $\left.\mathrm{H}_{2} \mathrm{CO}\right) / N\left(\right.$ para- $\left.\mathrm{H}_{2} \mathrm{CO}\right)$ ratio in several of the sources in our sample. Our measurements indicate that $N\left(\right.$ ortho- $\left.\mathrm{H}_{2} \mathrm{CO}\right) / N\left(\right.$ para- $\left.\mathrm{H}_{2} \mathrm{CO}\right)<3$ for most of our sources. When combined with the relatively high kinetic temperature in these objects, this $N$ (ortho$\left.\mathrm{H}_{2} \mathrm{CO}\right) / N\left(\right.$ para- $\mathrm{H}_{2} \mathrm{CO}$ ) ratio suggests that dust grains might play an active role in $\mathrm{H}_{2} \mathrm{CO}$ chemistry.

Subject headings: H II regions - ISM: molecules - ISM: clouds - molecular processes
\end{abstract}

\section{INTRODUCTION}

In order to study the process of star formation in dense molecular clouds, one must be able to make accurate measurements of the physical properties of these regions. Two of the most fundamental properties of a molecular cloud are its kinetic temperature and spatial density. Since the primary constituent of the molecular material, $\mathrm{H}_{2}$, cannot be measured directly, one must measure the properties of this material through its effect on other molecules in the region. Ideally, one would like this indirect molecular probe to possess several properties:

\footnotetext{
${ }^{1}$ The National Radio Astronomy Observatory is operated by Associated Universities, Inc., under cooperative agreement with the National Science Foundation.
}

1. Since it is important to be able to study a variety of starforming regions, one would prefer to use a molecular probe that is ubiquitous.

2. Since radiative transfer effects can often mask the physical properties of the densest locations within a star-forming region, a desirable property for the molecular emission would be simple radiative transfer (i.e., optically thin emission).

3. Chemical inhomogeneities can often lead to an inaccurate assessment of the physical properties within a star-forming region. For this reason one would like to use a molecular probe which possesses a simple chemical history.

4. Since the absolute calibration of molecular spectral lines at millimeter and submillimeter wavelengths is often difficult, it is useful to be able to measure several spectral lines from a given molecular probe with the same receiving system. 
5. The molecular energy levels for all molecules possess a coupled sensitivity to the kinetic temperature and spatial density in a molecular cloud. For this reason it is useful to find a molecule for which certain combinations of transitions have maximum sensitivity to just one of these physical parameters.

Formaldehyde has in the past been used to great advantage as a probe of spatial density in the cores of dense molecular clouds (cf. Wootten, Snell, \& Evans 1980; Mangum et al. 1990; Mangum, Wootten, \& Plambeck 1993). The unique emission properties of the " $K$-doublet" transitions of $\mathrm{H}_{2} \mathrm{CO}$, with their sensitivity to spatial densities $\gtrsim 10^{5} \mathrm{~cm}^{-3}$, have proved to be quite useful (Mangum et al. 1990; Mangum et al. $1993)$. Since $\mathrm{H}_{2} \mathrm{CO}$ is a slightly asymmetric rotor molecule, it should possess many of the kinetic temperature sensitivities inherent in purely symmetric rotor molecules. Several symmetric rotors, such as $\mathrm{NH}_{3}, \mathrm{CH}_{3} \mathrm{CN}$, and $\mathrm{CH}_{3} \mathrm{C}_{2} \mathrm{H}$, have been used as kinetic temperature indicators. Unfortunately, these molecules have found limited use as reliable probes because they have spatially variable abundances or complex excitation properties. Formaldehyde, on the other hand, pervades the interstellar medium at a relatively high abundance which does not vary substantially even in the chaotic Orion-KL region (Mangum et al. 1990). Since several formaldehyde transitions can be measured with the same telescope, and in some cases in the same spectrum, telescope-dependent calibration uncertainties can be eliminated. Therefore, formaldehyde should be an accurate probe of the kinetic temperature and spatial density in star-forming regions.

In the following we present a detailed analysis of the kinetic temperature and spatial density probing properties of $\mathrm{H}_{2} \mathrm{CO}$. Observations of many of the transitions studied in this analysis toward a sample of 11 star-forming regions are presented in $\S \S 2$ and 3 . In $\S 4$ we model the physical properties of the molecular clouds in our sample and study the utility of $\mathrm{H}_{2} \mathrm{CO}$ as a kinetic temperature and spatial density probe using the spherical large velocity gradient approximation. In $\S 5$ we discuss the importance of several approximations used in our model analysis. Our conclusions regarding the measurement of the physical properties of molecular clouds using $\mathrm{H}_{2} \mathrm{CO}$ are given in $\S 6$.

We also present three appendices which give general calculations of several important molecular emission characteristics. In Appendix A we derive a general expression which relates the kinetic temperature to the intensities of two transitions from different $K$-ladders within any symmetric or slightly asymmetric rotor molecule under the conditions of local thermodynamic equilibrium. Appendix B describes a general method which one can use to calculate the spontaneous emission coefficient for any rovibrational transition from a symmetric or slightly asymmetric rotor molecule. Appendix $\mathrm{C}$ uses a simple radiative transfer model to estimate the importance of any infrared excitation process to the overall excitation properties of a given molecule.

\section{OBSERVATIONS}

\section{1. $C S O$}

The Caltech Submillimeter Observatory (CSO) $10.4 \mathrm{~m}$ antenna located near the summit of Mauna Kea, Hawaii, was used over the period 1990 February $13-23$ to measure $\mathrm{H}_{2} \mathrm{CO}$ emission toward several sources. Additional measurements of OMC 2 IRS 4 were obtained during the period 1992 February $8-13$. The source positions are given in Table 1 , while Table 2 lists the $\mathrm{H}_{2} \mathrm{CO}$ transitions and frequencies measured toward these positions. The receiver employed for these measurements was a superconductor-insulator-superconductor (SIS) tunnel junction receiver developed by Ellison et al. (1989).

The single-sideband system temperature measured on the sky varied from $\sim 700$ to $\sim 2000 \mathrm{~K}$ over the observed elevation and frequency range. The weather was reasonably good throughout both observing periods $\left(\tau_{225} \lessgtr 0.2\right)$. The standard chopper-wheel calibration method (Ulich \& Haas 1976; Kutner \& Ulich 1981) was used to put the data on the $T_{A}^{*}$ temperature scale. Since many of our measurements were made at frequencies near the telluric $\mathrm{O}_{2}$ and $\mathrm{H}_{2} \mathrm{O}$ lines at 368.9 and $380.2 \mathrm{GHz}$, respectively, the atmospheric opacities in the signal and image sidebands of our double-sideband receiver are often not equal. Since the standard chopper-wheel method assumes equality for the signal and image sideband atmospheric opacities, a correction must be made to our $T_{A}^{*}$ scale. Using sky-dip measurements of the receiver voltage as a function of zenith angle, an estimate of the amount of precipitable water vapor present in the atmosphere was made several times during the observing run. These estimates of water vapor content were then applied to a model of atmospheric emission (Grossman 1989) to obtain the atmospheric opacity at the zenith for our observed frequencies. Then, assuming equal gain from the signal and image sideband of the receiver, the factor to correct for unequal sideband opacities that one must apply to the $T_{A}^{*}$ scale is given by

$$
\frac{1}{2}\left\{1+\exp \left[\sec (\mathrm{ZA})\left(\tau_{s}-\tau_{i}\right)\right]\right\} .
$$

$\mathrm{ZA}$ is the zenith angle of the observation, while $\tau_{s}$ and $\tau_{i}$ are the signal and image sideband atmospheric opacities, respectively. This correction factor ranged from $10 \%$ to $35 \%$ in our observations.

A further correction needs to be applied in order to take account of the forward scattering and spillover efficiency $\left(\eta_{\mathrm{fss}}\right)$ and coupling efficiency of the main beam to the source distribution $\left[\eta_{c}\right.$ (main beam) $]$. The product of these two efficiencies is often called the main-beam efficiency, $\eta_{\mathrm{mb}}=\eta_{\mathrm{fss}} \eta_{c}$ (main beam). Using measurements of Jupiter at $365 \mathrm{GHz}$ and assuming $T_{R}$ (Jupiter) $=168 \pm 6 \mathrm{~K}$ at this frequency (Griffin et al. 1986), a diffraction-limited FWHM beam width of 19", and a planetary diameter of $42^{\prime \prime}$, we measured $\eta_{\mathrm{mb}}$ (Jupiter) $=0.55 \pm$ 0.04 during both observing periods. Since we wish to combine measurements taken with comparable spatial resolution from two different millimeter-wave telescopes, we will assume that all sources are approximately equal to our calibrator source size $\left(\sim 40^{\prime \prime}\right)$ and will refer all line temperature measurements to the $T_{R}=T_{A}^{*} / \eta_{\mathrm{mb}}$ (Jupiter) scale. This assumption is justified given the fact that, as we find in $\S 4.5$, there appears to be no systematic difference between the physical properties derived for sources which are at distances $\$ 1 \mathrm{kpc}$ and those derived for sources at distances $\gtrsim 2 \mathrm{kpc}$.

The back end used was an acousto-optical spectrometer (AOS) consisting of $10241 \mathrm{MHz}$ channels over an instantaneous bandwidth of $500 \mathrm{MHz}$. Since the response of the AOS 
TABLE 1

Source Positions

\begin{tabular}{|c|c|c|c|c|}
\hline Source & $\alpha(1950)$ & $\delta(1950)$ & $\begin{array}{l}\text { Distance } \\
(\mathrm{pc})\end{array}$ & $\begin{array}{c}\text { Beam Size }^{\mathrm{a}} \\
(\mathrm{pc})\end{array}$ \\
\hline W3 IRS 4 & $02^{\mathrm{h}} 21^{\mathrm{m}} 43^{\mathrm{s}} .5$ & $61^{\circ} 52^{\prime} 49^{\prime \prime}$ & $2400^{\mathrm{b}}$ & 0.35 \\
\hline $\mathrm{W} 3(\mathrm{OH}) \ldots \ldots \ldots \ldots \ldots$ & $\begin{array}{lll}02 & 23 & 17.0\end{array}$ & 613853 & $2400^{\mathrm{b}}$ & 0.35 \\
\hline Orion-KL $\ldots \ldots \ldots \ldots$ & 053247.0 & $-05 \quad 2421$ & $480^{c}$ & 0.07 \\
\hline OMC-2 IRS $4 \ldots \ldots \ldots \ldots$ & 053259.6 & $-05 \quad 1132$ & $430^{d}$ & 0.06 \\
\hline NGC $2024 \ldots \ldots \ldots \ldots \ldots$ & $\begin{array}{lll}05 & 39 & 13.0\end{array}$ & -015628 & $415^{d}$ & 0.06 \\
\hline NGC 2071 IRS $1 \ldots \ldots$ & 054430.6 & 002042 & $390^{d}$ & 0.06 \\
\hline NGC 2264 IR $\ldots \ldots \ldots \ldots$ & 063825.0 & 093229 & $875^{\mathrm{e}}$ & 0.13 \\
\hline IRAS $16293-2422 \ldots \ldots$ & 162921.0 & -242213 & $160^{f}$ & 0.02 \\
\hline NGC $63341 \ldots \ldots \ldots \ldots$ & $1717 \quad 32.0$ & -354404 & $1740^{8}$ & 0.25 \\
\hline $\mathrm{G} 34.26+0.15 \ldots \ldots \ldots \ldots$ & 185046.2 & 011113 & $3800^{\mathrm{h}}$ & 0.56 \\
\hline DR $21(\mathrm{OH}) \ldots \ldots \ldots \ldots$ & $2037 \quad 14.2$ & 421210 & $3000^{\prime}$ & 0.44 \\
\hline
\end{tabular}

a This is the physical dimension of a $30^{\prime \prime}$ beam at the source distance.

${ }^{\mathrm{b}}$ Harris \& Wynn-Williams 1976.

c Genzel et al. 1981.

d Anthony-Twarog 1982.

${ }^{c}$ Mendoza \& Gómez 1980.

f Whittet 1974.

${ }^{8}$ Neckel 1978.

${ }^{\text {h }}$ Garay, Reid, \& Moran 1985.

i Campbell et al. 1982.

to the intermediate frequency is a function of the ambient temperature, we have applied a frequency calibration to our spectra by using a multiplied crystal oscillator to generate a frequency comb in the bandpass with peaks separated by 100 $\mathrm{MHz}$. Periodic measurements of the signal from this generator enabled us to measure the center channel and channel width of our spectra. The drift in the center channel was found to be as high as 3.5 channels $\left(\sim 1.8 \mathrm{~km} \mathrm{~s}^{-1}\right)$, while the variation in channel width was $\pm 0.0004 \mathrm{~km} \mathrm{~s}^{-1}$. Since the AOS is oversampled in frequency by approximately a factor of 2 , all spectra have been smoothed to a basic frequency resolution of 500 $\mathrm{MHz} / 512$ channels $\simeq 0.98 \mathrm{MHz}$ channel $^{-1}$. Pointing was checked frequently and found to vary by $\leqslant 5$ " between measurements.

For portions of two nights, the AOS was anomalously noisy, characterized by nonuniform baseline variations across many of the spectra. Most of our measurements of the $5_{05} \rightarrow 4_{04}$ and $5_{23} \rightarrow 4_{22}$ transitions were made during these periods, which for several sources leads to the anomalous $V_{\mathrm{LSR}}$ and $\Delta v$ of our Gaussian fits to these lines and the correspondingly large uncertainties associated with them. During subsequent observing runs we have checked the intensities of these transitions in several sources and found that this AOS instability has not significantly affected our measured transition intensities.

TABLE 2

OBSERVED $\mathrm{H}_{2} \mathrm{CO}$ TRANSITIONS

\begin{tabular}{|c|c|c|c|c|c|c|}
\hline Transition & $\begin{array}{l}\text { Frequency } \\
(\mathrm{MHz})\end{array}$ & $\begin{array}{c}E_{u} \\
(\mathrm{~K})\end{array}$ & $S$ & Telescope & $\theta_{\mathrm{mb}}$ & $\eta_{\mathrm{mb}}$ \\
\hline $3_{13} \rightarrow 2_{12} \ldots \ldots$ & 211211.468 & 32.1 & 2.6666 & NRAO & $30^{\prime \prime}$ & $0.45 \pm 0.02^{\mathrm{b}}$ \\
\hline $3_{03} \rightarrow 2_{02} \ldots \ldots$ & 218222.192 & 21.0 & 2.9994 & NRAO & 30 & $0.45 \pm 0.02^{\mathrm{b}}$ \\
\hline $3_{22} \rightarrow 2_{21} \ldots \ldots$ & 218475.632 & 68.1 & 1.6667 & NRAO & 30 & $0.45 \pm 0.02^{\mathrm{b}}$ \\
\hline $4_{32} \rightarrow 3_{31} \ldots \ldots$ & 291380.488 & 141.1 & 1.7500 & NRAO & 22 & $0.28 \pm 0.02^{\mathrm{c}}$ \\
\hline $4_{31} \rightarrow 3_{30} \ldots \ldots$ & 291384.264 & 141.1 & 1.7500 & NRAO & 22 & $0.28 \pm 0.02^{c}$ \\
\hline $5_{15} \rightarrow 4_{14} \ldots \ldots$ & 351768.645 & 62.5 & 4.7996 & CSO & 20 & $0.55 \pm 0.04^{\mathrm{d}}$ \\
\hline $5_{05} \rightarrow 4_{04} \ldots \ldots$ & 362736.048 & 52.4 & 4.9971 & CSO & 20 & $0.55 \pm 0.04^{\mathrm{d}}$ \\
\hline $5_{24} \rightarrow 4_{23} \ldots \ldots$ & 363945.894 & 99.6 & 4.1998 & CSO & 20 & $0.55 \pm 0.04^{\mathrm{d}}$ \\
\hline $5_{42} \rightarrow 4_{41} \ldots \ldots$ & 364103.249 & 240.9 & 1.8000 & CSO & 20 & $0.55 \pm 0.04^{\mathrm{d}}$ \\
\hline $5_{41} \rightarrow 4_{40} \ldots \ldots$ & 364103.249 & 240.9 & 1.8000 & $\mathrm{CSO}$ & 20 & $0.55 \pm 0.04^{\mathrm{d}}$ \\
\hline $5_{33} \rightarrow 4_{32} \ldots \ldots$ & 364275.141 & 158.5 & 3.2000 & CSO & 20 & $0.55 \pm 0.04^{\mathrm{d}}$ \\
\hline $5_{32} \rightarrow 4_{31} \ldots \ldots$ & 364288.884 & 158.5 & 3.2000 & $\mathrm{CSO}$ & 20 & $0.55 \pm 0.04^{\mathrm{d}}$ \\
\hline $5_{23} \rightarrow 4_{22} \ldots \ldots$ & 365363.428 & 99.7 & 4.1998 & $\mathrm{CSO}$ & 20 & $0.55 \pm 0.04^{\mathrm{d}}$ \\
\hline
\end{tabular}

${ }^{a}$ Frequencies from Cornet \& Winnewisser 1980.

${ }^{\mathrm{b}}$ Calculated using antenna temperature measurements of Jupiter $\left(\theta=38^{\prime \prime}\right.$; see $\left.\S 2.2\right)$.

${ }^{c}$ Extrapolated from Jupiter measurements at $218 \mathrm{GHz}$ (see $\S 2.2$ ).

${ }^{\mathrm{d}}$ Calculated using antenna temperature measurements of Jupiter $(\theta=41$ "; see $\S 2.1)$. 


\section{2. $N R A O$}

Observations of the 211-292 GHz transitions of $\mathrm{H}_{2} \mathrm{CO}$ were obtained during three separate observing sessions (1988 December 15-20, 1989 April 29 and 30, and 1990 March 10-13) using the NRAO $12 \mathrm{~m}$ telescope located on Kitt Peak, Arizona. The source positions are given in Table 1, while Table 2 lists the $\mathrm{H}_{2} \mathrm{CO}$ transitions and frequencies measured toward these positions. The receiver employed for these observations was a cooled dual-channel Schottky diode mixer receiver. The single-sideband system temperature measured on the sky varied from $1500 \mathrm{~K}$ at $211 \mathrm{GHz}$ to $7000 \mathrm{~K}$ at $291 \mathrm{GHz}$. Atmospheric opacity at $218 \mathrm{GHz}$ was measured frequently during these observing sessions and was found to be less than 0.4.

The chopper-wheel method was used for spectral line calibration. Unlike most millimeter-wavelength facilities, however, spectra at the $12 \mathrm{~m}$ telescope are corrected for the forward scattering and spillover efficiency $\eta_{\text {fss }}$, which puts them on the $T_{R}^{*}\left(=T_{A}^{*} / \eta_{\mathrm{fss}}\right)$ scale $\left(\eta_{\mathrm{fss}}=0.75\right.$ at $\left.230 \mathrm{GHz}\right)$. Using measurements of Jupiter at $218 \mathrm{GHz}$ and assuming $T_{R}$ (Jupiter) $=$ $171 \pm 14 \mathrm{~K}$ at this frequency (Griffin et al. 1986), a diffraction-limited FWHM beamwidth of 29", and a planetary diameter of 39", we measured a coupling efficiency for Jupiter, $\eta_{c}(\mathrm{Ju}-$ piter), of $0.60 \pm 0.02$. This efficiency is appropriate for our measurements in the $211-218 \mathrm{GHz}$ range but is not applicable at $291 \mathrm{GHz}$. At $291 \mathrm{GHz}$, the main-beam efficiency is $\sim 60 \%$ of its value at $218 \mathrm{GHz}$. Therefore, we estimate that $\eta_{c}(\mathrm{Ju}$ piter $)=0.37 \pm 0.02$ at $291 \mathrm{GHz}$. The diffraction-limited beamwidth at $291 \mathrm{GHz}$ is $21^{\prime \prime}$. We have applied these coupling efficiency measurements to our $T_{R}^{*}$-scale spectra in order to put all measurements on the radiation temperature $\left(T_{R}\right)$ scale. As was done with our CSO measurements, we assume that all source sizes are approximately equal to that of Jupiter.

The back end employed for these observations was a set of two dual-polarization 128 channel filter spectrometers which provided spectral resolutions of 0.25 and $1 \mathrm{MHz}$. By frequently checking the telescope pointing during each observing session, we estimate our pointing errors to be $₫ 5$.

\section{RESULTS}

Individual spectra for the measured transitions from each source are shown in Figures 1-11. The results from Gaussian fits to these measured spectra are given in Table 3. In order to compare transition intensities at similar spectral resolution, we have smoothed our $0.25 \mathrm{MHz}$ resolution NRAO measurements to the velocity resolution of our CSO measurements $\left(\sim 0.8 \mathrm{~km} \mathrm{~s}^{-1}\right)$. For only one object (OMC 2 IRS 4$)$ did this smoothing result in a dilution in transition intensity. Most of the spectra required only one Gaussian component. For most of the sources in Table 3, the LSR velocities from the individual transitions agree to within $\sim 1 \mathrm{~km} \mathrm{~s}^{-1}$, while the line width, $\Delta v$, can vary by as much as a factor of 4 . In several sources there appears to be a systematic variation in $\Delta v$ as a function of transition energy. Excluding the $5_{05} \rightarrow 4_{04}$ and $5_{23} \rightarrow 4_{22}$ transitions, which may have been broadened by AOS instability, OMC 2 IRS 4, NGC 2024, NGC 2071 IRS 1, and DR 21(OH) show $\mathrm{H}_{2} \mathrm{CO}$ line-width broadening by factors of 2-4 with increasing transition energy. The simplest explanation for such a trend is that higher excitation transitions are more sensitive to

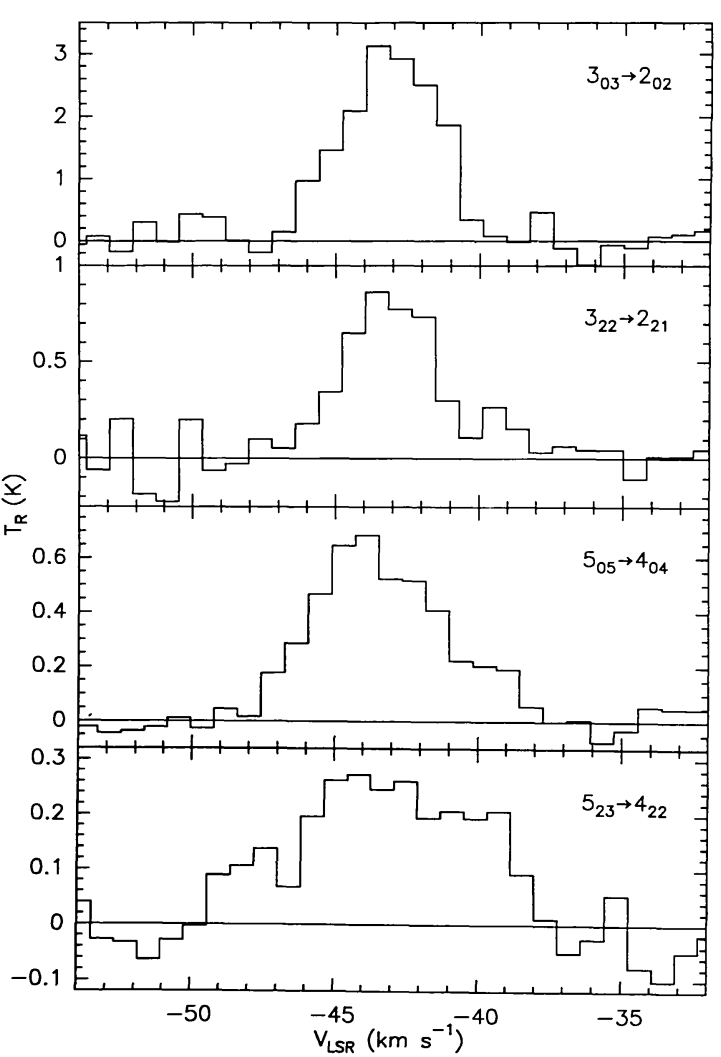

FIG. 1.-Para- $\mathrm{H}_{2} \mathrm{CO}$ spectra of W3 IRS 4

the warm, turbulent inner core region where young stars may be forming. Since in the analysis presented below we assume a constant-density isothermal cloud model which is dependent mainly upon the $\mathrm{H}_{2} \mathrm{CO}$ transition intensities, we do not address transition line-width variations in this paper. An understanding of line-width variations will require molecular cloud models which include radial variations in the spatial density, kinetic temperature, and velocity fields.

\section{ANALYSIS}

\section{1. $\mathrm{H}_{2} \mathrm{CO}$ as a Probe of Kinetic Temperature and Spatial Density}

$\mathrm{H}_{2} \mathrm{CO}$ is a proved tracer of the high-density environs of molecular clouds. It is ubiquitous: $\mathrm{H}_{2} \mathrm{CO}$ is associated with $80 \%$ of the H II regions surveyed by Downes et al. (1980), and possesses a large number of observationally accessible transitions from centimeter to far-infrared wavelengths. Because $\mathrm{H}_{2} \mathrm{CO}$ is a slightly asymmetric rotor molecule, each rotational level is split by this asymmetry into two energy levels. The energy levels can be designated by a total angular momentum quantum number, $J$; the projection of $J$ along the symmetry axis for a limiting prolate symmetric top, $K_{-1}$; and the projection of $J$ along the symmetry axis for a limiting oblate symmetric top, $K_{+1}$. The $\mathrm{H}_{2} \mathrm{CO}$ energy-level diagram is shown in Figure 12.

In the radiative excitation of a symmetric rotor molecule, dipole selection rules dictate that $\Delta K=0$. Transitions between energy levels with different $K$-values operate only through collisional excitation. Thus, a comparison between the energy-le- 


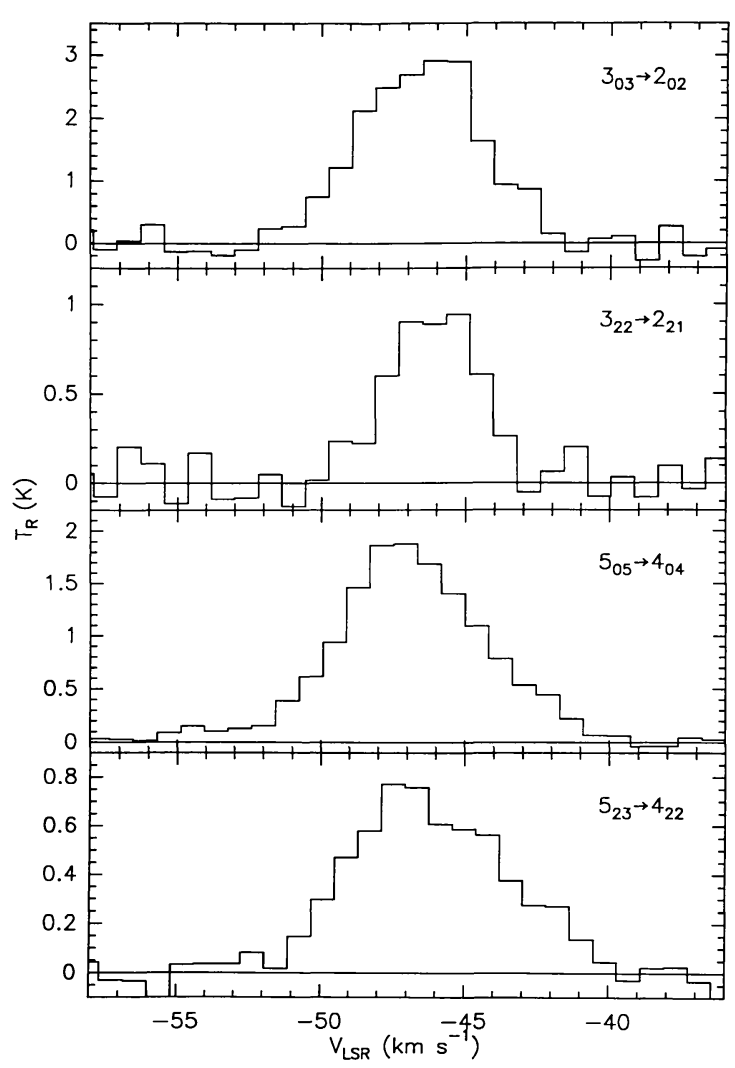

FIG. 2.-Para- $\mathrm{H}_{2} \mathrm{CO}$ spectra of $\mathrm{W} 3(\mathrm{OH})$

vel populations from different $K$-levels within the same symmetry species should be a direct measure of the kinetic temperature in the gas. Transitions involving the same $J$-levels which come from different $K$-levels are closely spaced in frequency. As noted above, the asymmetry in $\mathrm{H}_{2} \mathrm{CO}$ is very small $(\kappa=-0.96)$; it is structurally similar to a prolate symmetric rotor molecule $(\kappa=-1.0)$. Therefore, by measuring the intensities of two $K$-components from the same $\Delta J=1$ transition, one can obtain a measure of the kinetic temperature in a molecular cloud which is unaffected by relative pointing uncertainties, beam-size differences, and absolute calibration uncertainties.

In general, the relative population of a molecular energy level is determined by a coupled sensitivity to spatial density and kinetic temperature. Since the energy-level structure of the $\mathrm{H}_{2} \mathrm{CO}$ molecule allows one to decouple the excitation due to kinetic temperature, a direct measure of the spatial density in a molecular cloud can be obtained. In the following sections we will analyze the kinetic temperature and spatial density sensitivity of several $\mathrm{H}_{2} \mathrm{CO}$ transitions.

\section{2. $\mathrm{H}_{2} \mathrm{CO}$ Large Velocity Gradient Modeling Procedure}

In the following, we will use the spherical large velocity gradient (LVG) approximation to model the physical properties of molecular cloud cores. In the spherical LVG approximation, the molecular cloud is assumed to be a sphere with uniform spatial density, kinetic temperature, and molecular column density whose radiative transfer is governed by the local physical conditions in the cloud. Solutions to the coupled statistical equilibrium and radiative transfer equations in this approximation can be expressed in the form of transition radiation temperatures and optical depths.

In addition to a specification of the molecular spectrum,
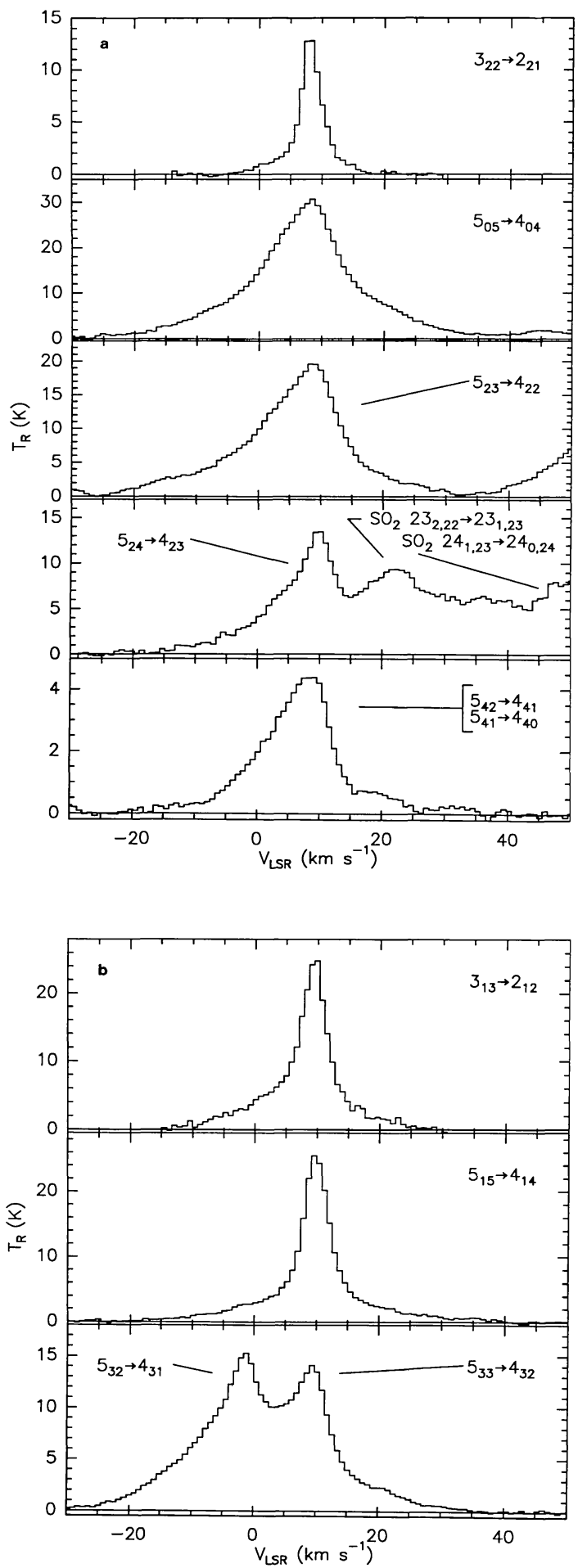

FIG. 3.-( $a$ ) Para- $\mathrm{H}_{2} \mathrm{CO}$ spectra of Orion-KL. (b) Ortho- $\mathrm{H}_{2} \mathrm{CO}$ spectra of Orion-KL. 

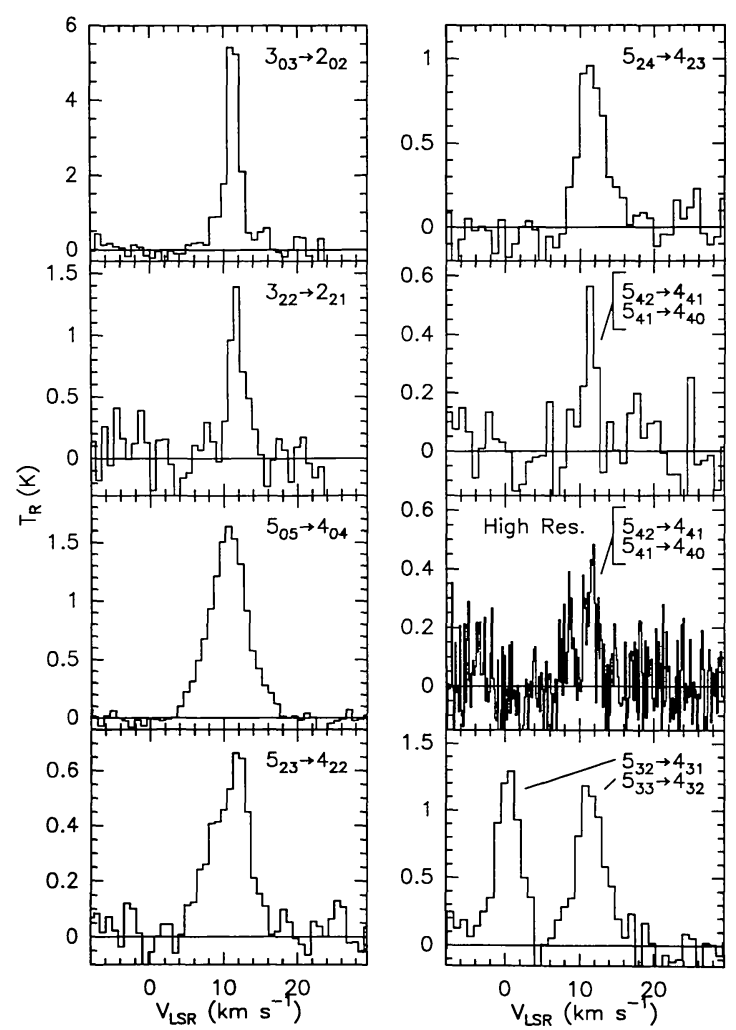

FIG. 4.-Para- and ortho- $\mathrm{H}_{2} \mathrm{CO}$ spectra of OMC-2 IRS 4. Note that for the $5_{42} \rightarrow 4_{41} / 5_{41} \rightarrow 4_{40}$ transition pair we show both low and high velocity resolution spectra.
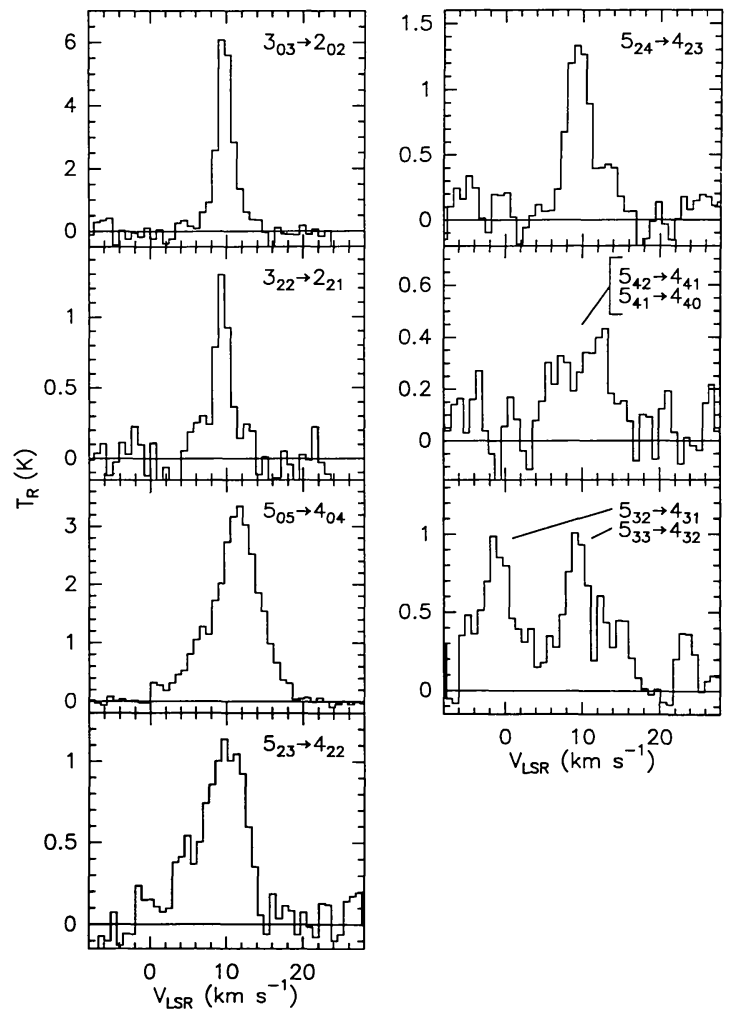

FIG. 6.-Para- and ortho- $\mathrm{H}_{2} \mathrm{CO}$ spectra of NGC 2071 IRS 1

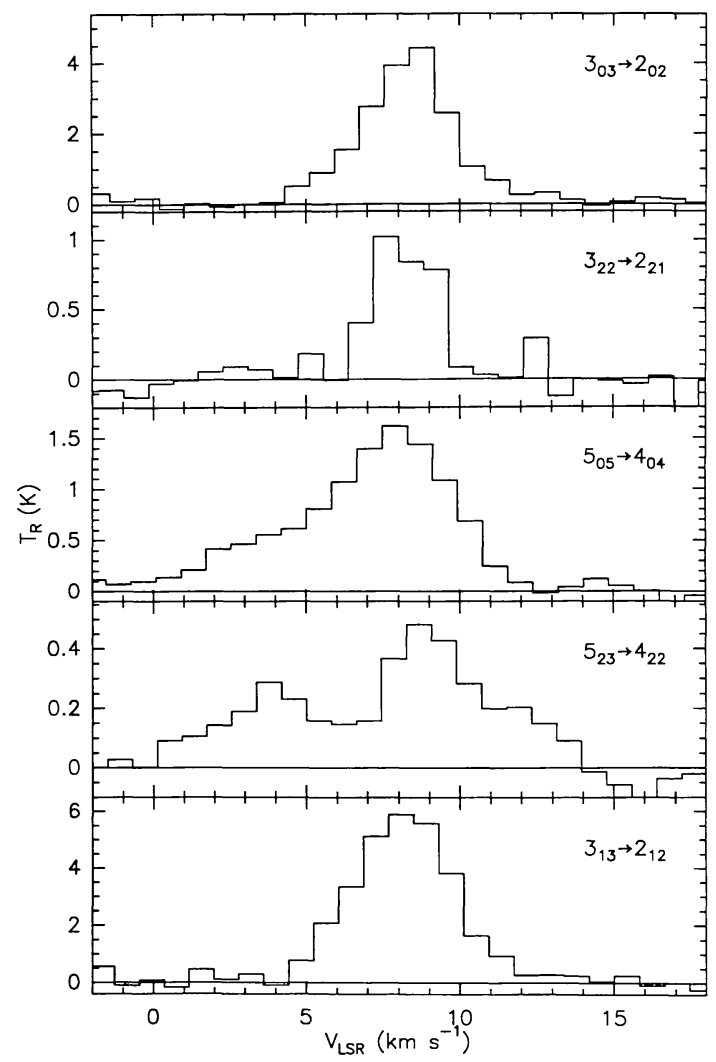

FIG. 7.-Para- and ortho- $\mathrm{H}_{2} \mathrm{CO}$ spectra of NGC 2264IR

FIG. 5.-Para- and ortho- $\mathrm{H}_{2} \mathrm{CO}$ spectra of NGC 2024

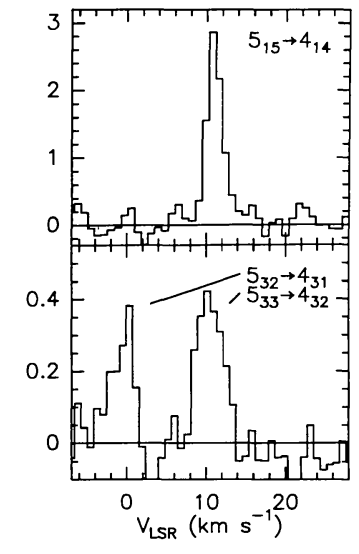




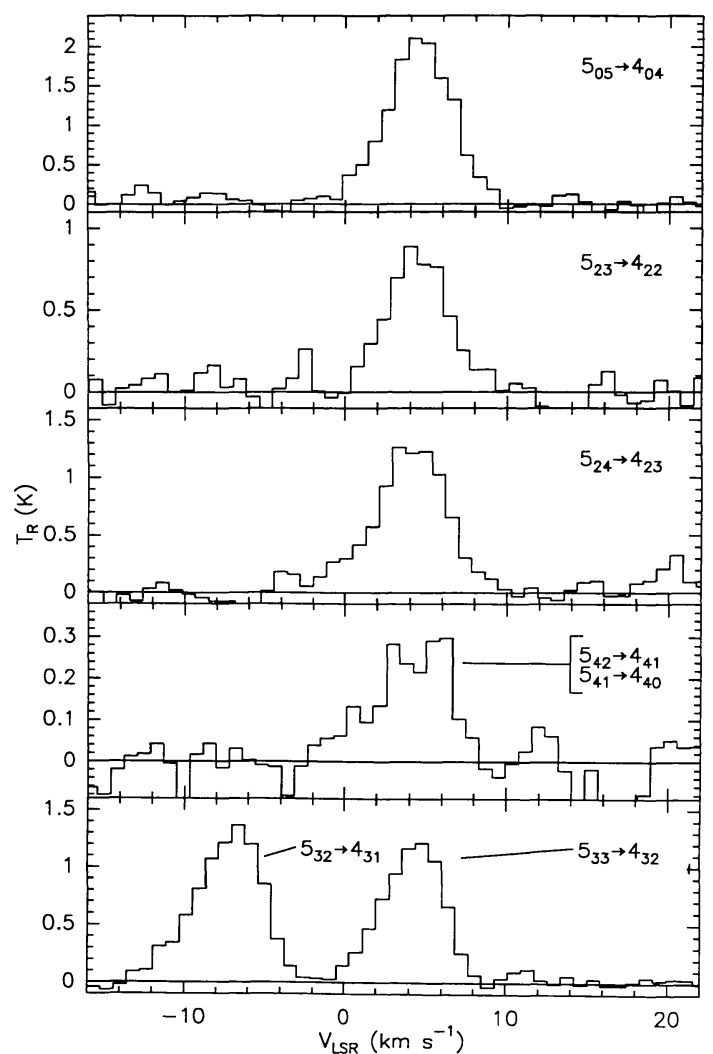

FIG. 8.-Para- and ortho- $\mathrm{H}_{2} \mathrm{CO}$ spectra of IRAS $16293-2422$

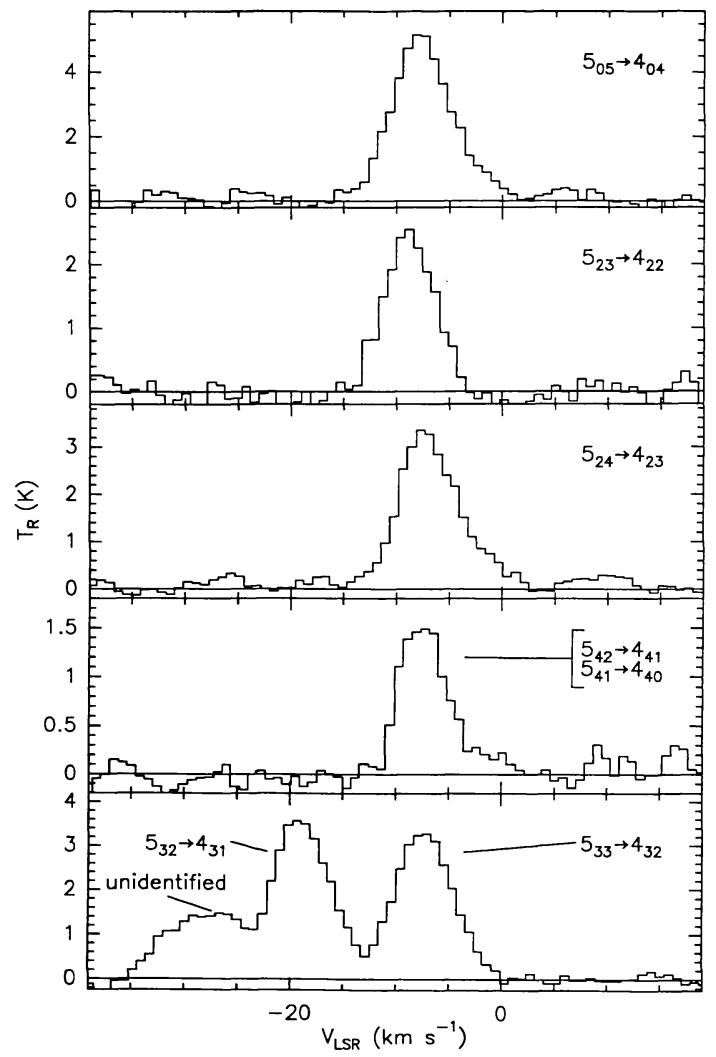

FIG. 9.-Para- and ortho- $\mathrm{H}_{2} \mathrm{CO}$ spectra of NGC 6334I

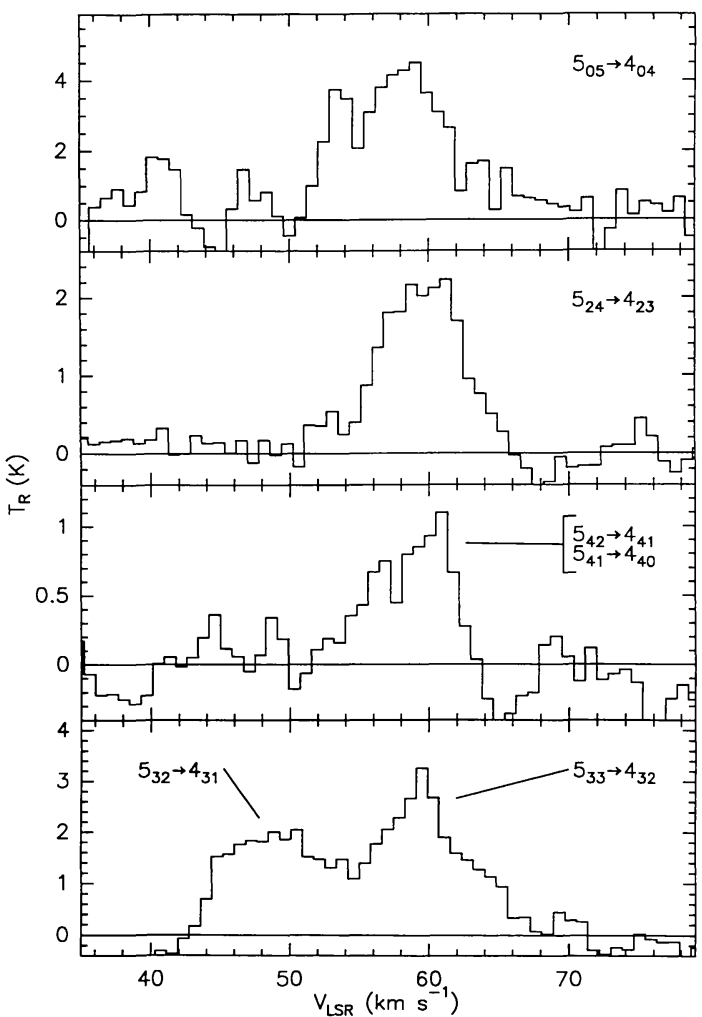

FIG. 10.-Para- and ortho- $\mathrm{H}_{2} \mathrm{CO}$ spectra of G34.26+0.15
LVG models require as input the collisional excitation rates. Green (1991) has calculated the rotational excitation of formaldehyde by collisions with $\mathrm{He}$ atoms. These new rates include the lowest 81 rotational levels of $\mathrm{H}_{2} \mathrm{CO}$ and have been caiculated up to kinetic temperatures of $300 \mathrm{~K}$. Because of the difficulty in specifying the interaction potential for $\mathrm{H}_{2} \mathrm{CO}$ and $\mathrm{H}_{2}$, these rates are calculated using $\mathrm{He}$ atoms as the collision partner. According to Green (1991), the relative efficiency of collisional excitation by $\mathrm{H}_{2}$ compared with $\mathrm{He}$ can be obtained from pressure-broadening data. Nerf (1975) has measured room temperature values for the broadening of $\mathrm{H}_{2} \mathrm{CO}$ microwave lines by $\mathrm{H}_{2}$ and $\mathrm{He}$ which indicate that $\mathrm{H}_{2} \mathrm{CO}-\mathrm{H}_{2}$ collision cross sections are $\sim 1$. 6 times larger than $\mathrm{H}_{2} \mathrm{CO}-\mathrm{He}$ collision cross sections. Collisional excitation rates are also a function of the relative collision velocities, which are proportional to the inverse square root of the masses of the particles. Therefore, since $\mathrm{He}$ has twice the mass of $\mathrm{H}_{2}$, collisional excitation due to $\mathrm{H}_{2}$ should be 2.2 times more effective than collisional excitation by $\mathrm{He}$. Green suggests that the calculated $\mathrm{H}_{2} \mathrm{CO}-\mathrm{He}$ rates be scaled uniformly by this factor, and we have done so in our model calculations. Green also points out that the $\mathrm{H}_{2} \mathrm{CO}-\mathrm{He}$ state-to-state rates are probably accurate to $\sim 50 \%$ and that total excitation rates are probably accurate to $\sim 20 \%$. Although we do not include this uncertainty in our model analysis, one should keep this in mind when interpreting $\mathrm{H}_{2} \mathrm{CO}$ LVG model results.

In the present analysis, we concentrate on models of the excitation of the para species of $\mathrm{H}_{2} \mathrm{CO}$. Since para- $\mathrm{H}_{2} \mathrm{CO}$ is $1-3$ times less abundant than ortho- $\mathrm{H}_{2} \mathrm{CO}$ (Kahane et al. 1984), 

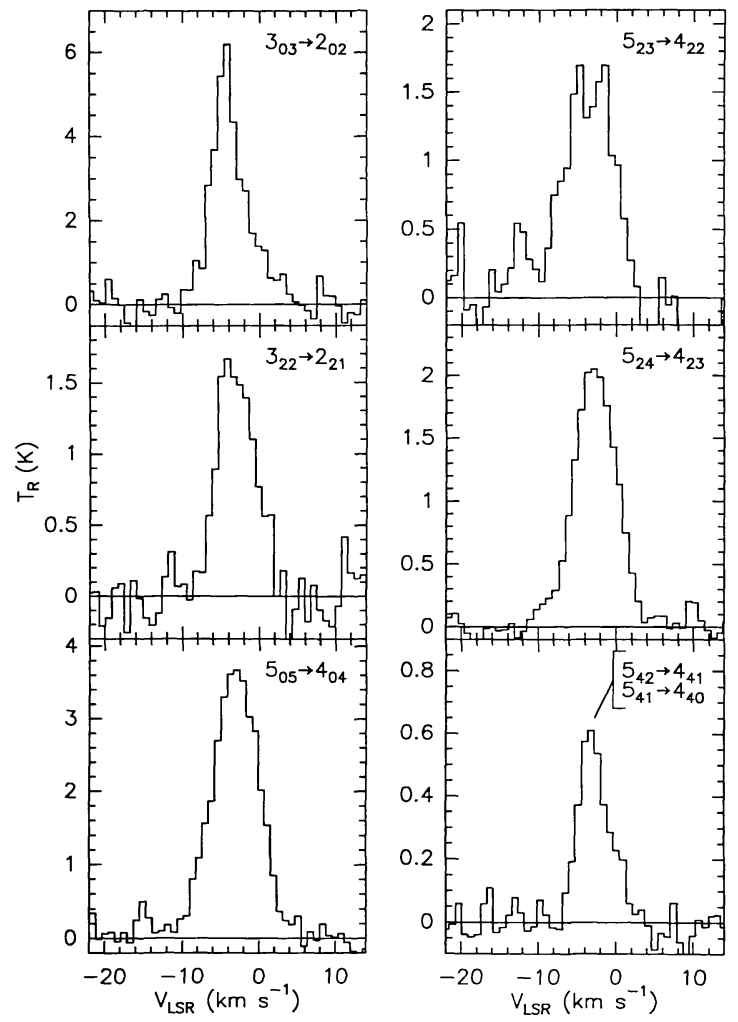

FIG. $11 a$

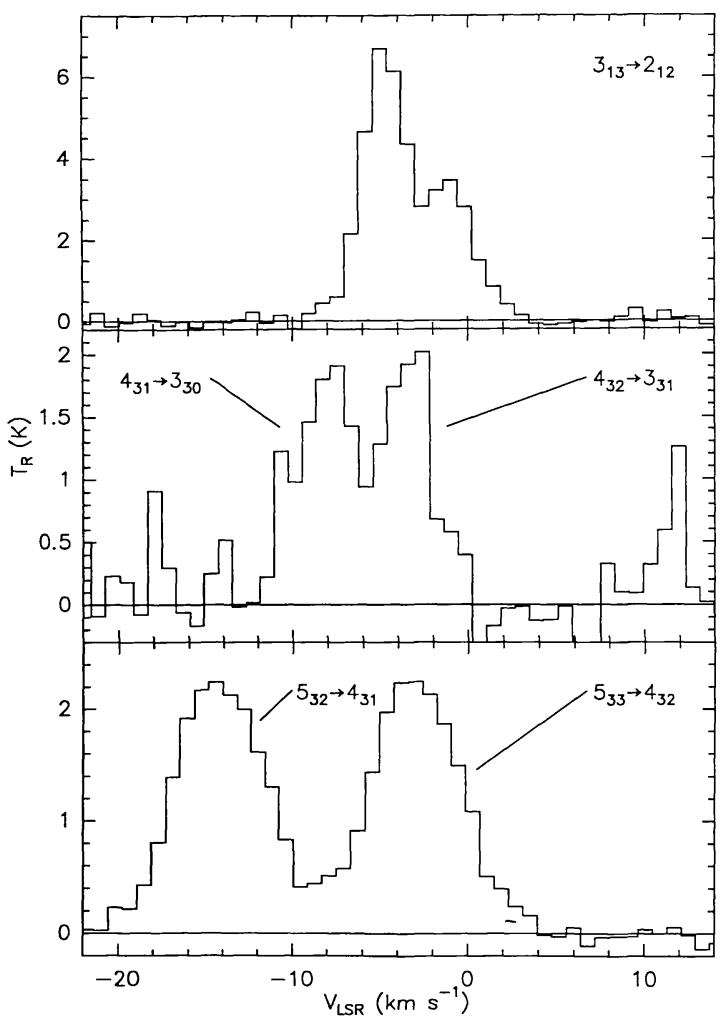

FIG. $11 b$

Fig. 11.-(a) Para- $\mathrm{H}_{2} \mathrm{CO}$ spectra of DR $21(\mathrm{OH})$. (b) Ortho- $\mathrm{H}_{2} \mathrm{CO}$ spectra of DR $21(\mathrm{OH})$.

uncertainties due to high transition optical depth are minimized. Our para- $\mathrm{H}_{2} \mathrm{CO}$ models include transitions up to $J=$ $12\left(E_{u}=270 \mathrm{~K}\right)$ in the $K_{-1}=0$ ladder, $J=11\left(E_{u}=280 \mathrm{~K}\right)$ in the $K_{-1}=2$ ladder, and $J=7\left(E_{u}=286 \mathrm{~K}\right)$ in the $K_{-1}=4$ ladder. We also present some results from our analysis of the excitation of ortho- $\mathrm{H}_{2} \mathrm{CO}$. These models include transitions up to $J=12\left(E_{u}=293 \mathrm{~K}\right)$ in the $K_{-1}=1$ ladder and $J=10$ $\left(E_{u}=299 \mathrm{~K}\right)$ in the $K_{-1}=3$ ladder.

In calculating the $\mathrm{H}_{2} \mathrm{CO}$ spectrum from these levels for both species, we include all allowed transitions, including the weak $\Delta K_{-1}=2$ transitions which exist due to the slight asymmetry of the molecule. In order to determine the importance of these $\Delta K_{-1}=2$ transitions to the LVG model-predicted radiation temperatures, we have compared our results with models which exclude these transitions. At low spatial density and / or kinetic temperature, exclusion of the $\Delta K_{-1}=2$ transitions produces $5 \%-25 \%$ increases in the predicted radiation temperatures. At high spatial density and/or kinetic temperature, exclusion of the $\Delta K_{-1}=2$ transitions produces 5\%-20\% decreases in predicted radiation temperatures for the lower excitation transitions and 5\%-50\% increases in predicted radiation temperatures for the higher excitation transitions. In most cases, the larger increases and decreases in predicted radiation temperature occur in transitions whose level populations and intensities are relatively small. Therefore, we estimate that exclusion of the $\Delta K_{-1}=2$ transitions in models of $\mathrm{H}_{2} \mathrm{CO}$ excitation under interstellar conditions results in an error of at most $20 \%$. Note that this error estimate does not include the effects of far-infrared absorption through the $\Delta K_{-1}=2$ transitions.
We will analyze the general problem of infrared absorption in $\mathrm{H}_{2} \mathrm{CO}$ in $\$ 5.2$ and Appendix C.

In the following model analyses, we will use three basic methods for visualizing the solutions to the spatial density, kinetic temperature, and $\mathrm{H}_{2} \mathrm{CO}$ species column density per unit line width in each molecular cloud. In $\S \S 4.3$ and 4.4 we investigate the kinetic temperature and spatial density sensitivity of various $\mathrm{H}_{2} \mathrm{CO}$ transitions. We show these sensitivities by plotting contours of predicted $T_{R}$ and $T_{R}$ ratios as a function of spatial density, kinetic temperature, and $\mathrm{H}_{2} \mathrm{CO}$ species column density per unit line width. In $\S 4.5$ we compare all of the observed radiation temperatures for a given molecular cloud with those predicted by the LVG models to determine the region in $n\left(\mathrm{H}_{2}\right)-T_{K}-N\left(\right.$ species- $\left.\mathrm{H}_{2} \mathrm{CO}\right) / \Delta v$ parameter space, which contains the most probable solutions to these variables.

\subsection{Kinetic Temperature Measurement}

\subsubsection{LVG Solution}

As noted in $\S 4.1$, by comparing the intensities of two $K$ components from the same $\Delta J=1$ transition, one can obtain a measure of the kinetic temperature in a molecular cloud. Receiver instability, variations in telescope efficiency as a function of frequency, and pointing instabilities can often dominate the uncertainties in molecular spectral line radiation temperature measurements at millimeter and submillimeter wavelengths. Therefore, accurate measurements of the kinetic temperature can best be made when the transitions used can be 
TABLE 3

$\mathrm{H}_{2} \mathrm{CO}$ MEASUREMENT Results

\begin{tabular}{|c|c|c|c|c|}
\hline $\begin{array}{l}\text { Source and } \\
\text { Species }\end{array}$ & Transition & $\begin{array}{l}\text { Peak } T_{R}{ }^{\mathrm{a}} \\
\quad(\mathrm{K})\end{array}$ & $\begin{array}{c}V_{\mathrm{LSR}}^{\mathrm{b}} \\
\left(\mathrm{km} \mathrm{s}^{-1}\right)\end{array}$ & $\begin{array}{c}\Delta v \\
\left(\mathrm{~km} \mathrm{~s}^{-1}\right)\end{array}$ \\
\hline \multicolumn{5}{|l|}{ W3 IRS4: } \\
\hline \multirow[t]{5}{*}{ para $\ldots \ldots \ldots \ldots$} & \multirow{5}{*}{$\left.\begin{array}{l}3_{03} \rightarrow 2_{02} \\
3_{22} \rightarrow 2_{21} \\
5_{05} \rightarrow 4_{04} \\
5_{23} \rightarrow 4_{22} \\
5_{24} \rightarrow 4_{23} \\
5_{42} \rightarrow 4_{41} \\
5_{41} \rightarrow 4_{40}\end{array}\right\}$} & $\begin{array}{l}3.10 \pm 0.32 \\
0.85+0.19\end{array}$ & $-43.10 \pm 0.08$ & $3.87 \pm 0.19$ \\
\hline & & $\begin{array}{l}0.85 \pm 0.19 \\
0.64 \pm 0.07\end{array}$ & $-43.63 \pm 0.73$ & $\begin{array}{l}3.18 \pm 0.32 \\
5.33 \pm 2.0\end{array}$ \\
\hline & & $0.26 \pm 0.07$ & $-43.35 \pm 0.77$ & $7.24 \pm 2.0$ \\
\hline & & $<0.53$ & $\ldots$ & $\ldots$ \\
\hline & & $<0.53$ & $\cdots$ & $\cdots$ \\
\hline \multirow[t]{2}{*}{ ortho $\ldots \ldots \ldots$} & $5_{32} \rightarrow 4_{31}$ & $<0.53$ & $\cdots$ & $\cdots$ \\
\hline & $5_{33} \rightarrow 4_{32}$ & $<0.53$ & $\cdots$ & $\cdots$ \\
\hline \multirow{4}{*}{ para...$\ldots \ldots \ldots$} & $3_{03} \rightarrow 2_{02}$ & $2.97 \pm 0.32$ & \multicolumn{2}{|c|}{ W3(OH): } \\
\hline & $3_{22} \rightarrow 2_{21}$ & $1.00 \pm 0.24$ & $-46.10 \pm 0.09$ & $3.54 \pm 0.20$ \\
\hline & $5_{05} \rightarrow 4_{04}$ & $1.83 \pm 0.14$ & $-46.95 \pm 0.74$ & $5.77 \pm 0.43$ \\
\hline & $5_{23} \rightarrow 4_{22}$ & $0.73 \pm 0.08$ & $-46.40 \pm 0.73$ & $6.43 \pm 0.29$ \\
\hline \multirow{15}{*}{$\begin{array}{l}\text { Orion-KL: } \\
\text { para } \ldots . . .\end{array}$} & \multirow{4}{*}{$3_{22} \rightarrow 2_{21}$} & & & \\
\hline & & $0.93 \pm 0.20$ & $4.0^{\mathrm{c}}$ & $10.0^{\mathrm{c}}$ \\
\hline & & $11.62 \pm 0.44$ & $8.27 \pm 0.03$ & $3.56 \pm 0.08$ \\
\hline & & $0.75 \pm 0.20$ & $8.8^{\mathrm{c}}$ & $18.0^{c}$ \\
\hline & \multirow{2}{*}{$5_{05} \rightarrow 4_{04}^{d}$} & $15.87^{\mathrm{c}}$ & $4.5^{\mathrm{c}}$ & $10.0^{\mathrm{c}}$ \\
\hline & & $16.26 \pm 1.20$ & $9.4^{\mathrm{c}}$ & $5.0^{\mathrm{c}}$ \\
\hline & \multirow{4}{*}{$5_{23} \rightarrow 4_{22}^{d}$} & $6.54^{c}$ & $7.5^{\mathrm{c}}$ & $28.0^{\mathrm{c}}$ \\
\hline & & $10.27 \pm 0.79$ & $4.0^{c}$ & $10.0^{\mathrm{c}}$ \\
\hline & & $10.92 \pm 0.84$ & $9.9^{\mathrm{c}}$ & $5.5^{\mathrm{c}}$ \\
\hline & & $4.56 \pm 0.42$ & $8.0^{\circ}$ & $28.0^{\mathrm{c}}$ \\
\hline & \multirow{2}{*}{$5_{24} \rightarrow 4_{23}^{e}$} & $4.87 \pm 0.45$ & $4.0^{c}$ & $10.0^{\mathrm{c}}$ \\
\hline & & $\begin{array}{l}9.94 \pm 0.77 \\
2.38 \pm 0.32\end{array}$ & $\begin{array}{l}9.9^{\mathrm{c}} \\
8.0^{\mathrm{c}}\end{array}$ & $\begin{array}{r}5.5^{\mathrm{c}} \\
28.0^{\mathrm{c}}\end{array}$ \\
\hline & \multirow{3}{*}{$\left.\begin{array}{rl}5_{42} & \rightarrow 4_{41} \\
5_{41} & \rightarrow \\
40\end{array}\right\}$} & $210+014$ & $40^{c}$ & 20.0 \\
\hline & & $2.10 \pm 0.14$ & $4.0^{\circ}$ & $10.0^{2}$ \\
\hline & & $2.54 \pm 0.15$ & $9.4^{\mathrm{c}}$ & $6.0^{\mathrm{c}}$ \\
\hline \multirow{12}{*}{ ortho $\ldots \ldots \ldots$} & \multirow{3}{*}{$3_{13} \rightarrow 2_{12}$} & $0.88 \pm 0.12$ & $8.0^{c}$ & $28.0^{\mathrm{c}}$ \\
\hline & & $1.78 \pm 0.59$ & $4.0^{\mathrm{c}}$ & $10.0^{\mathrm{c}}$ \\
\hline & & $18.67 \pm 0.85$ & $9.43 \pm 0.03$ & $4.19 \pm 0.09$ \\
\hline & \multirow{3}{*}{$5_{15} \rightarrow 4_{14}$} & $5.15 \pm 0.61$ & $8.0^{\mathrm{c}}$ & $20.0^{c}$ \\
\hline & & $0.82 \pm 0.23$ & $4.5^{\mathrm{c}}$ & $10.0^{\mathrm{c}}$ \\
\hline & & $21.45 \pm 1.58$ & $9.94 \pm 0.04$ & $4.6^{\mathrm{c}}$ \\
\hline & \multirow{3}{*}{$5_{32} \rightarrow 4_{31}$} & $\begin{array}{c}3.62 \pm 0.34 \\
3.8^{c}\end{array}$ & $\begin{array}{l}8.8^{\mathrm{c}} \\
4.0^{\mathrm{c}}\end{array}$ & $\begin{array}{l}30.0^{c} \\
10.0^{c}\end{array}$ \\
\hline & & $8.25 \pm 0.61$ & $9.9^{c}$ & $5.5^{\mathrm{c}}$ \\
\hline & & $3.8^{\mathrm{c}}$ & $8.0^{c}$ & $28.0^{\mathrm{c}}$ \\
\hline & \multirow[t]{3}{*}{$5_{33} \rightarrow 4_{32}$} & $3.8^{\mathrm{c}}$ & $4.0^{c}$ & $10.0^{c}$ \\
\hline & & $8.6^{\mathrm{c}}$ & $9.9^{c}$ & $5.5^{\mathrm{c}}$ \\
\hline & & $3.8^{\mathrm{c}}$ & $8.0^{c}$ & $28.0^{\mathrm{c}}$ \\
\hline \multirow{6}{*}{$\begin{array}{l}\text { OMC-2 IRS 4: } \\
\text { para } \ldots \ldots \ldots \ldots \ldots\end{array}$} & & & & \\
\hline & $3_{22} \rightarrow 2_{21}$ & $1.33 \pm 0.16$ & $\begin{array}{l}11.37 \pm 0.00 \\
11.71 \pm 0.10\end{array}$ & $\begin{array}{l}1.88 \pm 0.13 \\
2.23 \pm 0.26\end{array}$ \\
\hline & $5_{05} \rightarrow 4_{04}$ & $1.57 \pm 0.13$ & $10.57 \pm 0.72$ & $6.05 \pm 0.17$ \\
\hline & $5_{23} \rightarrow 4_{22}$ & $0.60 \pm 0.09$ & $10.97 \pm 0.74$ & $6.24 \pm 0.42$ \\
\hline & $5_{24} \rightarrow 4_{23}$ & $0.98 \pm 0.16$ & $11.51 \pm 0.17$ & $4.04 \pm 0.39$ \\
\hline & $\left.\begin{array}{l}5_{42} \rightarrow 4_{41} \\
5_{41} \rightarrow 4\end{array}\right\}$ & $0.34 \pm 0.11$ & $11.25 \pm 0.30$ & $3.5^{\mathrm{c}}$ \\
\hline ortho & $5_{32} \rightarrow 4_{31}$ & $0.96 \pm 0.16$ & $11.14 \pm 0.20$ & $4.60 \pm 0.50$ \\
\hline & $5_{33} \rightarrow 4_{32}$ & $0.85 \pm 0.16$ & $11.43 \pm 0.16$ & $3.82 \pm 0.38$ \\
\hline NGC 2024: & & & & \\
\hline para $\ldots$. & $3_{03} \rightarrow 2_{02}$ & $4.17 \pm 0.54$ & $10.89 \pm 0.06$ & $1.95 \pm 0.16$ \\
\hline & $3_{22} \rightarrow 2_{21}$ & $1.50 \pm 0.22$ & $10.80 \pm 0.11$ & $1.91 \pm 0.37$ \\
\hline & $5_{05} \rightarrow 4_{04}$ & $1.01 \pm 0.09$ & $10.30 \pm 0.72$ & $4.31 \pm 0.22$ \\
\hline & $5_{23} \rightarrow 4_{22}$ & $0.38 \pm 0.07$ & $11.01 \pm 0.76$ & $5.17 \pm 0.76$ \\
\hline & & $<0.40$ & $\ldots$ & $\ldots$ \\
\hline & $\left.\begin{array}{l}J_{42} \rightarrow 4_{41} \\
5_{41} \rightarrow 4_{40}\end{array}\right\}$ & $<0.48$ & $\cdots$ & $\cdots$ \\
\hline ortho ... & $5_{15} \rightarrow 4_{14}$ & $2.78 \pm 0.26$ & $10.94 \pm 0.07$ & $2.42 \pm 0.15$ \\
\hline & $5_{32} \rightarrow 4_{31}$ & $0.32 \pm 0.09$ & $10.88 \pm 0.38$ & $3.37 \pm 0.56$ \\
\hline & $5_{33} \rightarrow 4_{32}$ & $0.43 \pm 0.09$ & $10.46 \pm 0.35$ & $3.74 \pm 0.31$ \\
\hline
\end{tabular}


TABLE 3-Continued

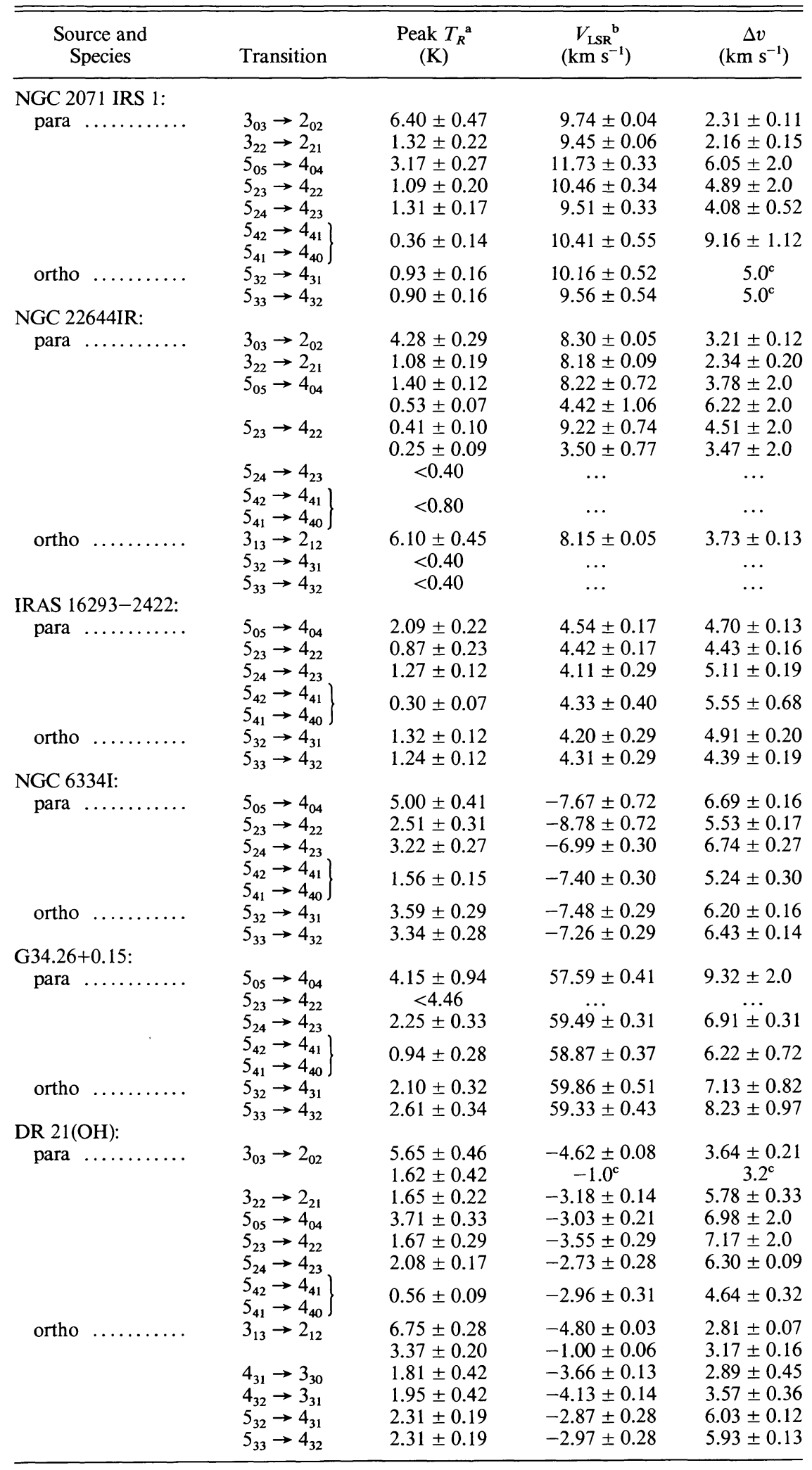

${ }^{a}$ Corrected for telescope efficiency and sideband optical depth inequality (see $\S 2$ ). All upper limits are given as $3 \sigma$.

${ }^{\mathrm{b}}$ Uncertainty includes contribution due to AOS drift.

${ }^{c}$ Quantity fixed in Gaussian fit.

d Measurements contaminated with unidentified lines.

${ }^{\mathrm{e}}$ Partially blended with $\mathrm{SO}_{2} 24_{1,23} \rightarrow 24_{0,24}$ and $23_{2,22} \rightarrow 23_{1,23}$. 


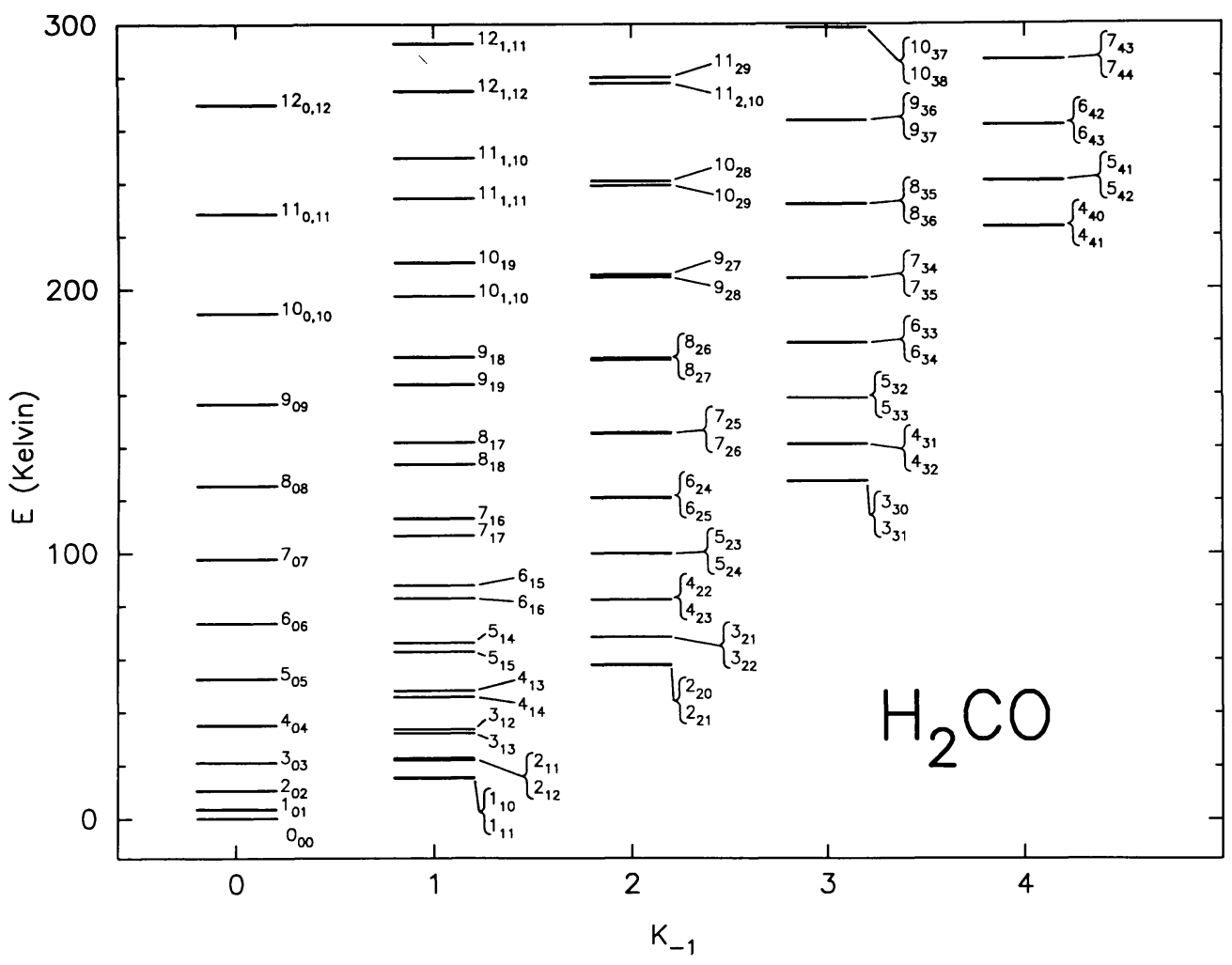

FIG. 12. $-\mathrm{H}_{2} \mathrm{CO}$ energy-level diagram

obtained with the same telescope and/or receiver. Several of the $\mathrm{H}_{2} \mathrm{CO}$ transitions listed in Table 2 meet these criteria.

From the para- $\mathrm{H}_{2} \mathrm{CO}$ transitions listed in Table 2, the following radiation temperature ratios should be calibration-independent monitors of the kinetic temperature in molecular clouds: $T_{R}\left(3_{03} \rightarrow 2_{02}\right) / T_{R}\left(3_{22} \rightarrow 2_{21}\right), T_{R}\left(5_{05} \rightarrow 4_{04}\right) / T_{R}\left(5_{23} \rightarrow 4_{22}\right)$, and $T_{R}\left(5_{24} \rightarrow 4_{23}\right) / T_{R}\left(5_{41} \rightarrow 4_{40}\right)$, or, equivalently, $T_{R}\left(5_{24} \rightarrow\right.$ $\left.4_{23}\right) / T_{R}\left(5_{42} \rightarrow 4_{41}\right)$. To this list we can add the $T_{R}\left(4_{04} \rightarrow\right.$ $\left.3_{03}\right) / T_{R}\left(4_{23} \rightarrow 3_{22}\right)$ ratio. The $3_{03} \rightarrow 2_{02}$ and $3_{22} \rightarrow 2_{21}$ transitions can be measured with the same receiver and, in some cases, within the same spectrum. The $4_{04} \rightarrow 3_{03}(290623.405$ $\mathrm{MHz})$ and $4_{23} \rightarrow 3_{22}(291237.780 \mathrm{MHz})$ transitions are closely spaced in frequency and can be measured using the same receiver. The $5_{05} \rightarrow 4_{04}$ and $5_{23} \rightarrow 4_{22}$ transitions can also be measured simultaneously and, in a double-sideband receiver with a $1.4 \mathrm{GHz}$ intermediate frequency, within the same spectrum. The $5_{24} \rightarrow 4_{23}$ and $5_{41} \rightarrow 4_{40}$ transitions are the easiest of these three transition pairs to measure simultaneously, since they can be measured within the same receiver sideband (and are therefore unaffected by sideband gain ratio uncertainties). From the ortho species of $\mathrm{H}_{2} \mathrm{CO}$ the $T_{R}\left(4_{14} \rightarrow 3_{13}\right) / T_{R}\left(4_{32} \rightarrow\right.$ $\left.3_{31}\right)$ and $T_{R}\left(5_{15} \rightarrow 4_{14}\right) / T_{R}\left(5_{33} \rightarrow 4_{32}\right)$ (or, equivalently, $T_{R}\left(5_{15} \rightarrow 4_{14}\right) / T_{R}\left(5_{32} \rightarrow 4_{31}\right)$ radiation temperature ratios should be reliable probes of the kinetic temperature. All three pairs can be measured with the same receiving system.

Figures 13 and 14 show LVG model predictions of the behavior of these radiation temperature ratios as a function of spatial density $\left[n\left(\mathrm{H}_{2}\right)\right]$ and kinetic temperature $\left(T_{K}\right)$ for representative values of the para- and ortho- $\mathrm{H}_{2} \mathrm{CO}$ column density per unit line width. Also indicated on these diagrams is the upperstate energy $\left(E_{u}\right)$ of the highest excitation transition. For all four para- $\mathrm{H}_{2} \mathrm{CO}$ ratios the opacity in the lower-lying transition becomes large when $N\left(\right.$ para- $\left.\mathrm{H}_{2} \mathrm{CO}\right) / \Delta v \gtrsim 10^{14} \mathrm{~cm}^{-2}(\mathrm{~km}$ $\left.\mathrm{s}^{-1}\right)^{-1}$, which causes the radiation temperature ratio to become dependent upon the column density. The sensitivity to para- $\mathrm{H}_{2} \mathrm{CO}$ column density over a representative range for the $T_{R}\left(3_{03} \rightarrow 2_{02}\right) / T_{R}\left(3_{22} \rightarrow 2_{21}\right)$ and $T_{R}\left(5_{05} \rightarrow 4_{04}\right) / T_{R}\left(5_{23} \rightarrow\right.$ $4_{22}$ ) ratios is shown in Figure 13b. It is apparent from Figures 13 and 14 that for $T_{K} \gtrsim E_{u}$ all three ratios become strongly dependent upon $n\left(\mathrm{H}_{2}\right)$. This behavior is due to the fact that as $T_{K}$ becomes larger than $E_{u}$ in a given transition ratio, most of the level population in the molecule is in the energy levels above $E_{u}$. For the $T_{R}\left(3_{03} \rightarrow 2_{02}\right) / T_{R}\left(3_{22} \rightarrow 2_{21}\right), T_{R}\left(4_{14} \rightarrow\right.$ $\left.3_{13}\right) / T_{R}\left(4_{32} \rightarrow 3_{31}\right)$, and $T_{R}\left(5_{24} \rightarrow 4_{23}\right) / T_{R}\left(5_{41} \rightarrow 4_{40}\right)$ ratios, this sensitivity to $n\left(\mathrm{H}_{2}\right)$ actually sets in before $T_{K}$ becomes greater than $E_{u}$. This occurs because the bottom energy levels of the $3_{22} \rightarrow 2_{21}, 4_{32} \rightarrow 3_{31} / 4_{31} \rightarrow 3_{30}$, and $5_{42} \rightarrow 4_{41} / 5_{41} \rightarrow 4_{40}$ transitions are at the bottom energy levels of the $K_{-1}=2,3$, and 4 ladders, respectively. Under certain sets of physical conditions population can pile up in the bottom levels of these transitions. Quantifying this behavior, we note the following:

1. At kinetic temperatures $\$ 50 \mathrm{~K}$ and spatial densities $\$ 10^{5.5} \mathrm{~cm}^{-3}$ most of the population within the $K_{-1}=2$ ladder piles up in the $2_{21}$ (ground-state) level. Then, for $T_{K}>50 \mathrm{~K}$ and $n\left(\mathrm{H}_{2}\right)=10^{5.5}-10^{7.0} \mathrm{~cm}^{-3}$, with increasing spatial density and / or kinetic temperature the population in the $2_{21}$ level falls much faster than the population in the $3_{22}$ level. This leads to a 

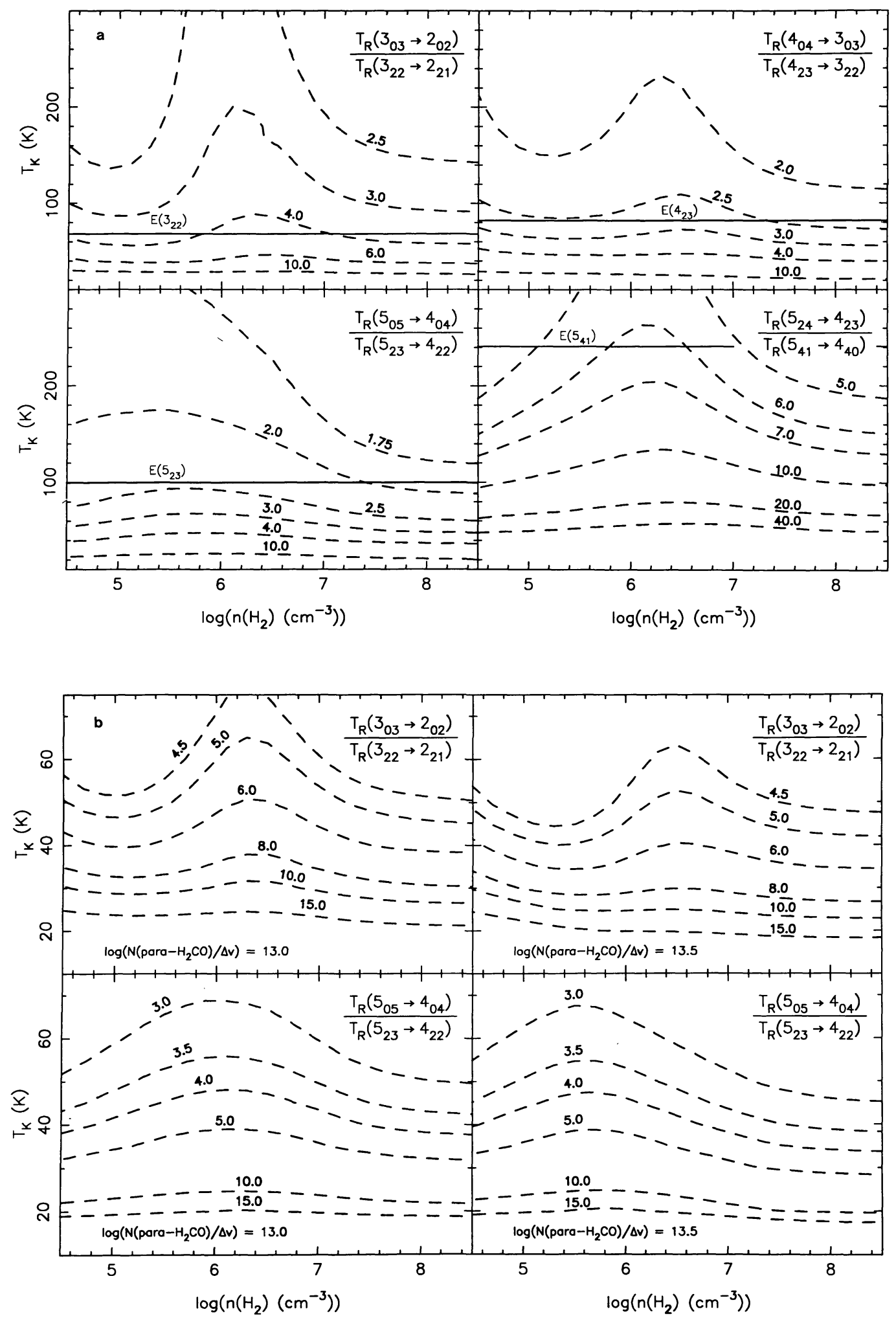

FIG. 13.- (a) LVG model calculations of the kinetic temperature sensitivity of four para- $\mathrm{H}_{2} \mathrm{CO}$ transition ratios. The calculations were made assuming $\log \left[N\left(\right.\right.$ para- $\left.\left.\mathrm{H}_{2} \mathrm{CO}\right) / \Delta \mathrm{v}\right]=13.3 \mathrm{~cm}^{-2}\left(\mathrm{~km} \mathrm{~s}^{-1}\right)^{-1}$, which is the mean value from our best-fit LVG model solutions (see Table 5). The state energy for the highest lying energy level is indicated for each ratio. $(b) \mathrm{LVG}$ model calculations of the kinetic temperature sensitivity of two of the para- $\mathrm{H}_{2} \mathrm{CO}$ transition ratios from $(a)$. This diagram shows the behavior of each ratio as a function of spatial density at the two column densities indicated. In comparison with $(a)$, we display the radiation temperature ratios over a range in $T_{K}$ in which they are the most sensitive. 


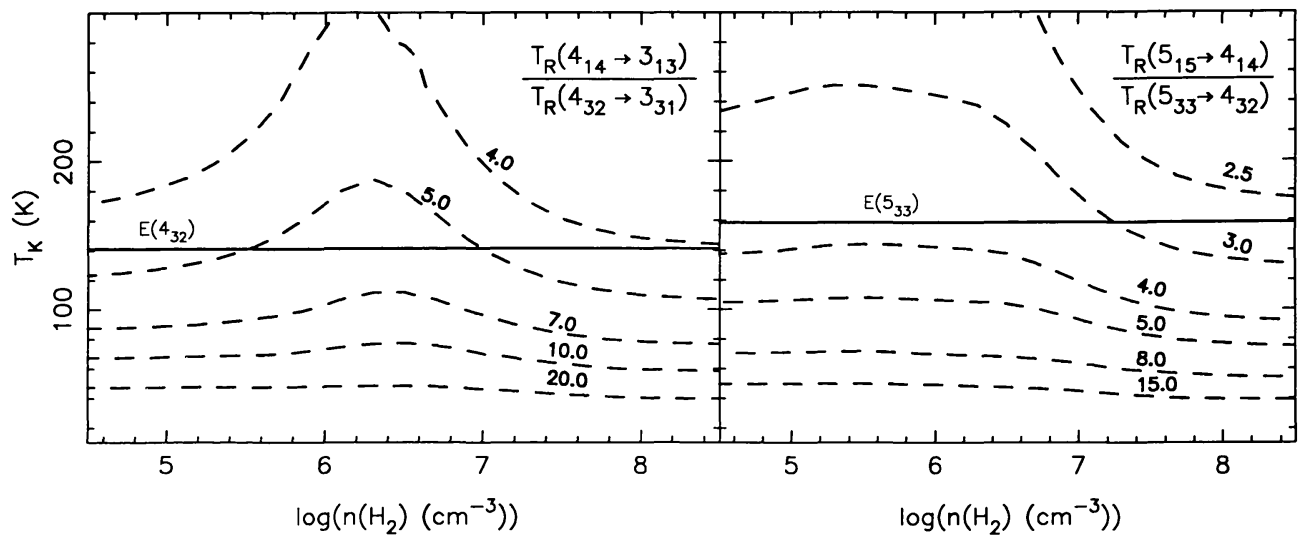

FIG. 14.-LVG model calculations of the kinetic temperature sensitivity of two ortho- $\mathrm{H}_{2} \mathrm{CO}$ transition ratios. The calculations were made assuming $\log \left[N\left(\right.\right.$ ortho- $\left.\left.\mathrm{H}_{2} \mathrm{CO}\right) / \Delta v\right]=13.6 \mathrm{~cm}^{-2}\left(\mathrm{~km} \mathrm{~s}^{-1}\right)^{-1}$, which is typical of the molecular clouds in our sample. The state energy for the highest lying energy level is indicated for each ratio.

condition where $T_{\text {ex }}\left(3_{22} \rightarrow 2_{21}\right)$ rises much faster than $T_{\text {ex }}\left(3_{03} \rightarrow 2_{02}\right)$, causing the "bump" in $T_{R}\left(3_{03} \rightarrow 2_{02}\right) /$ $T_{R}\left(3_{22} \rightarrow 2_{21}\right)$ apparent in Figure 13.

2. At kinetic temperatures $\$ 100 \mathrm{~K}$ and spatial densities $\$ 10^{5.5} \mathrm{~cm}^{-3}$ most of the population within the $K_{-1}=3$ ladder piles up in the $3_{30}$ and $3_{31}$ (ground-state) levels. Then, for $T_{K}>$ $100 \mathrm{~K}$ and $n\left(\mathrm{H}_{2}\right)=10^{5.5}-10^{7.0} \mathrm{~cm}^{-3}$, with increasing spatial density and/or kinetic temperature the populations in the $3_{30}$ and $3_{31}$ levels fall much faster than the populations in the $4_{31}$ and $4_{32}$ levels. This leads to a condition where $T_{\text {ex }}\left(4_{31} \rightarrow 3_{30}\right)$ and $T_{\text {ex }}\left(4_{32} \rightarrow 3_{31}\right)$ rise much faster than $T_{\text {ex }}\left(4_{14} \rightarrow 3_{13}\right)$, causing the "bump" in $T_{R}\left(4_{14} \rightarrow 3_{13}\right) / T_{R}\left(4_{32} \rightarrow 3_{31}\right)$ apparent in Figure 14.

3. At kinetic temperatures $\$ 100 \mathrm{~K}$ and spatial densities $\$ 10^{5.5} \mathrm{~cm}^{-3}$ most of the population within the $K_{-1}=4$ ladder piles up in the $4_{40}$ and $4_{41}$ (ground-state) levels. Then, for $T_{K}>$ $100 \mathrm{~K}$ and $n\left(\mathrm{H}_{2}\right)=10^{5.5}-10^{7.0} \mathrm{~cm}^{-3}$, with increasing spatial density and/or kinetic temperature the populations in the $4_{40}$ and $4_{41}$ levels fall much faster than the populations in the $5_{41}$ and $5_{42}$ levels. This leads to a condition where $T_{\text {ex }}\left(5_{41} \rightarrow 4_{40}\right)$ and $T_{\mathrm{ex}}\left(5_{42} \rightarrow 4_{41}\right)$ rise much faster than $T_{\mathrm{ex}}\left(5_{24} \rightarrow 4_{23}\right)$, which partially accounts for the "bump" in $T_{R}\left(5_{24} \rightarrow 4_{23}\right) /$ $T_{R}\left(5_{41} \rightarrow 4_{40}\right)$ found in Figure 13. The large population in energy levels above $5_{24}$ in the $K_{-1}=2$ ladder contributes more to the variation of this radiation temperature ratio with $n\left(\mathrm{H}_{2}\right)$. By the time a significant number of photons populate the $4_{40}$ level, there is also a significant number of photons in the $9_{27}$ level, eight levels above $5_{24}$ (see Fig. 12). This means that for $n\left(\mathrm{H}_{2}\right)<10^{6.0}, T_{\text {ex }}\left(5_{41} \rightarrow 4_{40}\right) \gg T_{\text {ex }}\left(5_{24} \rightarrow 4_{23}\right)$, which causes constancy of the $T_{R}\left(5_{24} \rightarrow 4_{23}\right) / T_{R}\left(5_{41} \rightarrow 4_{40}\right)$ ratio to break down long before $T_{K}$ reaches $E_{u}$.

This detailed understanding of the behavior of these radiation temperature ratios under changes in $n\left(\mathrm{H}_{2}\right), T_{K}$, and $N\left(\right.$ para- $\left.\mathrm{H}_{2} \mathrm{CO}\right) / \Delta v$ or $N\left(\right.$ ortho- $\left.\mathrm{H}_{2} \mathrm{CO}\right) / \Delta v$ allows us to derive the rules of thumb given below for using these $\mathrm{H}_{2} \mathrm{CO}$ transition ratios as probes of kinetic temperature in molecular cloud cores. Independent of the spatial density $\left[n\left(\mathrm{H}_{2}\right)\right]$ in a molecular cloud core, $T_{K}$ is measured with an uncertainty of $\$ 25 \%$ using the following:

1. $T_{R}\left(3_{03} \rightarrow 2_{02}\right) / T_{R}\left(3_{22} \rightarrow 2_{21}\right)$ for measurements of $T_{K} \lesssim$ $50 \mathrm{~K}$ with $N\left(\right.$ para- $\left.\mathrm{H}_{2} \mathrm{CO}\right) / \Delta v \lesssim 10^{13.5} \mathrm{~cm}^{-2}\left(\mathrm{~km} \mathrm{~s}^{-1}\right)^{-1}$.
2. $T_{R}\left(4_{04} \rightarrow 3_{03}\right) / T_{R}\left(4_{23} \rightarrow 3_{22}\right)$ for measurements of $T_{K} \lesssim$ $75 \mathrm{~K}$ with $N\left(\right.$ para- $\left.\mathrm{H}_{2} \mathrm{CO}\right) / \Delta v \lesssim 10^{13.75} \mathrm{~cm}^{-2}\left(\mathrm{~km} \mathrm{~s}^{-1}\right)^{-1}$.

3. $T_{R}\left(5_{05} \rightarrow 4_{04}\right) / T_{R}\left(5_{23} \rightarrow 4_{22}\right)$ for measurements of $T_{K} \lessgtr$ $75 \mathrm{~K}$ with $N\left(\right.$ para- $\left.\mathrm{H}_{2} \mathrm{CO}\right) / \Delta v \lesssim 10^{14.0} \mathrm{~cm}^{-2}\left(\mathrm{~km} \mathrm{~s}^{-1}\right)^{-1}$.

4. $T_{R}\left(5_{24} \rightarrow 4_{23}\right) / T_{R}\left(5_{41} \rightarrow 4_{40}\right)$ for measurements of $T_{K} \lesssim$ $150 \mathrm{~K}$ with $N\left(\right.$ para- $\left.\mathrm{H}_{2} \mathrm{CO}\right) / \Delta v \lesssim 10^{14.5} \mathrm{~cm}^{-2}\left(\mathrm{~km} \mathrm{~s}^{-1}\right)^{-1}$.

5. $T_{R}\left(4_{14} \rightarrow 3_{13}\right) / T_{R}\left(4_{32} \rightarrow 3_{31}\right)$ for measurements of $T_{K} \lesssim$ $100 \mathrm{~K}$ with $N\left(\right.$ ortho- $\left.\mathrm{H}_{2} \mathrm{CO}\right) / \Delta v \lesssim 10^{13.75} \mathrm{~cm}^{-2}\left(\mathrm{~km} \mathrm{~s}^{-1}\right)^{-1}$.

6. $T_{R}\left(5_{15} \rightarrow 4_{14}\right) / T_{R}\left(5_{33} \rightarrow 4_{32}\right)$ for measurements of $T_{K} \lesssim$ $100 \mathrm{~K}$ with $N\left(\right.$ ortho- $\left.\mathrm{H}_{2} \mathrm{CO}\right) / \Delta v \lesssim 10^{14.0} \mathrm{~cm}^{-2}\left(\mathrm{~km} \mathrm{~s}^{-1}\right)^{-1}$.

Therefore, one can use radiation temperature measurements of just two transitions of $\mathrm{H}_{2} \mathrm{CO}$, which can in many cases be obtained simultaneously, to derive the kinetic temperature in a molecular cloud. The radiation temperature ratio so obtained, and therefore the kinetic temperature derived from that ratio, are relatively independent of the spatial density and species column density in the region.

A priori information regarding the kinetic temperature in a molecular cloud may not always be available. Since the radiation temperature ratios analyzed above are sensitive over certain ranges in kinetic temperature and $\mathrm{H}_{2} \mathrm{CO}$ column density, it is advantageous to measure more than one ratio to increase the accuracy of a $T_{K}$ measurement. To illustrate this point, consider W3 IRS 4 . We have measured the $T_{R}\left(3_{03} \rightarrow 2_{02}\right) /$ $T_{R}\left(3_{22} \rightarrow 2_{21}\right)$ and $T_{R}\left(5_{05} \rightarrow 4_{04}\right) / T_{R}\left(5_{23} \rightarrow 4_{22}\right)$ radiation temperature ratios, obtaining values of $3.65 \pm 0.88$ and $2.45 \pm$ 0.69 , respectively. Referring to Figure 13, neither one of these ratio measurements alone does a good job of constraining the kinetic temperature. Taken together, though, these two ratios indicate that $T_{K} \simeq 75 \mathrm{~K}$ toward W3 IRS 4 and also allow one to estimate the spatial density in the region ( in order to obtain the most accurate measurement of the kinetic temperature in a molecular cloud core, one should analyze several of the radiation temperature ratios listed above.

\subsubsection{LTE Solution}

In Appendix A we derive an equation which uses the radiation temperature measurements from two $K$-components within the same $\Delta J=1$ transition to derive the kinetic temperature in a molecular cloud assuming local thermodynamic equilibrium (LTE). There are many approximations and assump- 
TABLE 4

LTE SOLUTIONS To $T_{K}$

\begin{tabular}{|c|c|c|c|c|c|c|}
\hline $\begin{array}{c}T_{K} \\
(\mathbf{K})\end{array}$ & $\frac{\int J_{v}\left(T_{R}\left(3_{03} \rightarrow 2_{02}\right)\right] d v}{\int J_{v}\left(T_{R}\left(3_{22} \rightarrow 2_{21}\right)\right] d v}$ & $\frac{\int J_{v}\left(T_{R}\left(4_{04} \rightarrow 3_{03}\right)\right] d v}{\int J_{v}\left(T_{R}\left(4_{23} \rightarrow 3_{22}\right)\right] d v}$ & $\frac{\int J_{v}\left(T_{R}\left(5_{05} \rightarrow 4_{04}\right)\right] d v}{\int J_{v}\left(T_{R}\left(5_{23} \rightarrow 4_{22}\right)\right] d v}$ & $\frac{\int J_{\nu}\left(T_{R}\left(5_{24} \rightarrow 4_{23}\right)\right] d v}{\int J_{\nu}\left(T_{R}\left(5_{41} \rightarrow 4_{40}\right)\right] d v}$ & $\frac{\int J_{\nu}\left(T_{R}\left(4_{14} \rightarrow 3_{13}\right)\right] d v}{\int J_{\nu}\left(T_{R}\left(4_{32} \rightarrow 3_{31}\right)\right] d v}$ & $\frac{\int J_{\nu}\left(T_{R}\left(5_{15} \rightarrow 4_{14}\right)\right] d v}{\int J_{\nu}\left(T_{R}\left(5_{33} \rightarrow 4_{32}\right)\right] d v}$ \\
\hline $10 \ldots \ldots$ & 201.50 & 145.96 & 137.05 & $\ldots$ & & \\
\hline $20 \ldots \ldots$ & 19.04 & 13.77 & 12.82 & $\ldots$ & 248.25 & 182.63 \\
\hline $30 \ldots \ldots$ & 8.67 & 6.27 & 5.82 & 254.40 & 50.58 & 36.85 \\
\hline $40 \ldots \ldots$ & 5.85 & 4.23 & 3.92 & 78.45 & 22.83 & 16.55 \\
\hline $50 \ldots \ldots$ & 4.62 & 3.34 & 3.09 & 38.73 & 14.17 & 10.24 \\
\hline $60 \ldots \ldots$ & 3.95 & 2.85 & 2.64 & 24.19 & 10.31 & 7.43 \\
\hline $70 \ldots \ldots$ & 3.53 & 2.55 & 2.36 & 17.28 & 8.21 & 5.91 \\
\hline $80 \ldots \ldots$ & 3.25 & 2.34 & 2.17 & 13.43 & 6.92 & 4.98 \\
\hline $90 \ldots \ldots$ & 3.04 & 2.20 & 2.03 & 11.04 & 6.06 & 4.36 \\
\hline $100 \ldots \ldots$ & 2.88 & 2.08 & 1.93 & 9.44 & 5.45 & 3.92 \\
\hline $120 \ldots \ldots$ & 2.67 & 1.93 & 1.78 & 7.46 & 4.65 & 3.34 \\
\hline $140 \ldots \ldots$ & 2.52 & 1.82 & 1.68 & 6.30 & 4.15 & 2.98 \\
\hline $160 \ldots \ldots$ & 2.42 & 1.75 & 1.61 & 5.56 & 3.81 & 2.73 \\
\hline $180 \ldots \ldots$ & 2.34 & 1.69 & 1.56 & 5.04 & 3.57 & 2.56 \\
\hline $200 \ldots \ldots$ & 2.28 & 1.65 & 1.52 & 4.66 & 3.38 & 2.42 \\
\hline $250 \ldots \ldots$ & 2.17 & 1.57 & 1.45 & 4.05 & 3.08 & 2.20 \\
\hline $300 \ldots \ldots$ & 2.11 & 1.52 & 1.40 & 3.68 & 2.89 & 2.07 \\
\hline
\end{tabular}


tions which go into the derivation of this equation, the most important of which are (1) that the transitions used are optically thin, (2) that the excitation temperatures of the two transitions are equal, and (3) that the transitions are thermalized. In our LVG analysis of $\S 4.3 .1$, we found that the para- $\mathrm{H}_{2} \mathrm{CO}$ transitions in that study become thermalized only at relatively high spatial densities of $n\left(\mathrm{H}_{2}\right) \gtrsim 10^{6.5} \mathrm{~cm}^{-3}$. In Table 4 we list the radiation temperature ratios derived from the LTE equation of Appendix A for the transitions studied in $\S 4.3 .1$. Comparison with Figures 13 and 14 indicates that there is good agreement between the LTE- and LVG-derived kinetic temperatures under the optically thin, high-density conditions inherent in the LTE assumptions. In Appendix A we also found that, within the conditions under which the LTE approximation is valid, the kinetic temperatures derived from this relation are uncertain by $\$ 30 \%$. This is similar to the inherent uncertainties encountered in the LVG solution, but the LVG solution is a much more general result, as it is applicable over a much wider range of physical conditions.

\subsection{Spatial Density Measurement}

Measurement of the spatial density $\left[n\left(\mathrm{H}_{2}\right)\right]$ in a molecular cloud core is much more difficult than measuring the kinetic temperature. Since a comparison between the intensities of two $\Delta J=1$ transitions from the same $K$-ladder is required, the ratio is sensitive not only to $n\left(\mathrm{H}_{2}\right)$ but also to $T_{K}$. For this reason, one must first specify the kinetic temperature before trying to derive $n\left(\mathrm{H}_{2}\right)$ using such a radiation temperature ra- tio. It is also rarely possible to measure both transitions in this ratio using the same receiver, since they lie at very different frequencies (see Table 2). This makes it necessary to obtain good absolute calibration on the individual transitions in this ratio, which is often difficult to do at millimeter and submillimeter wavelengths.

In Figure 15 we show LVG model predictions of the spatial density sensitivity of the $T_{R}\left(3_{03} \rightarrow 2_{02}\right) / T_{R}\left(5_{05} \rightarrow 4_{04}\right)$ and $T_{R}\left(3_{22} \rightarrow 2_{21}\right) / T_{R}\left(5_{24} \rightarrow 4_{23}\right)$ radiation temperature ratios. To show how any density-probing ratio is also sensitive to the kinetic temperature, we display these ratios at two values of $T_{K}$. From this analysis, we can state the "rules of thumb" given below for using these $\mathrm{H}_{2} \mathrm{CO}$ transition ratios as probes of spatial density in molecular cloud cores. With a specification of the kinetic temperature in a molecular cloud core and for $n\left(\mathrm{H}_{2}\right) \gtrsim 10^{5} \mathrm{~cm}^{-3}, n\left(\mathrm{H}_{2}\right)$ is measured with an uncertainty of $\$ 25 \%$ using the following:

1. $T_{R}\left(3_{03} \rightarrow 2_{02}\right) / T_{R}\left(5_{05} \rightarrow 4_{04}\right)$ for $N\left(\right.$ para $\left.-\mathrm{H}_{2} \mathrm{CO}\right) / \Delta v \lesssim$ $10^{13.5} \mathrm{~cm}^{-2}\left(\mathrm{~km} \mathrm{~s}^{-1}\right)^{-1}$.

2. $T_{R}\left(3_{22} \rightarrow 2_{21}\right) / T_{R}\left(5_{24} \rightarrow 4_{23}\right)$ for $N\left(\right.$ para- $\left.\mathrm{H}_{2} \mathrm{CO}\right) / \Delta v \lessgtr$ $10^{14.0} \mathrm{~cm}^{-2}\left(\mathrm{~km} \mathrm{~s}^{-1}\right)^{-1}$.

Therefore, once the kinetic temperature has been determined, one can use radiation temperature measurements of two appropriately chosen $\mathrm{H}_{2} \mathrm{CO}$ transitions to derive the spatial density in a molecular cloud core. Because these two transitions cannot in general be measured with the same receiving system, absolute calibration of these measurements is necessary.

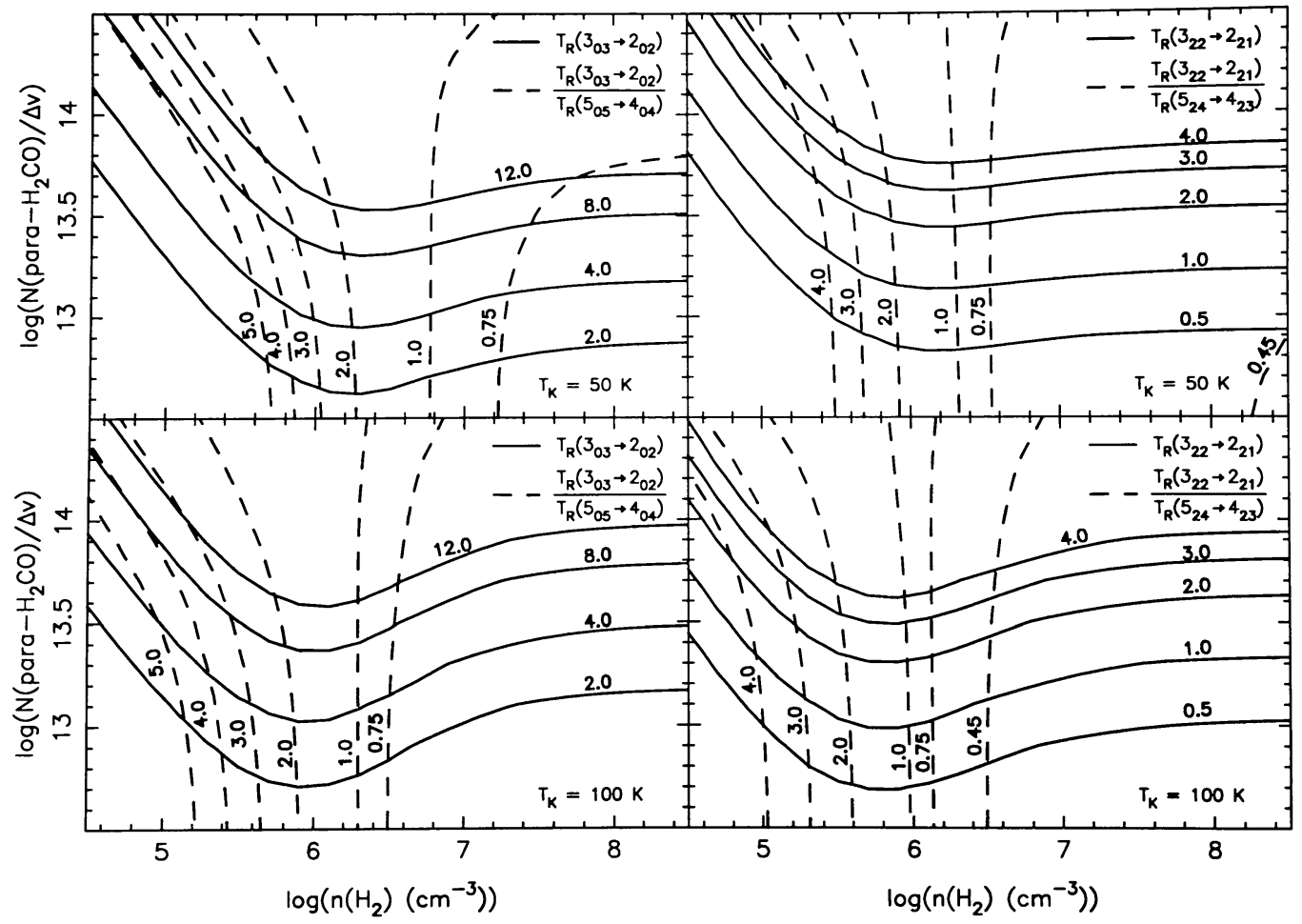

FIG. 15.-LVG model calculations of the spatial density sensitivity of two para- $\mathrm{H}_{2} \mathrm{CO}$ transition ratios. The upper two panels show this sensitivity for an assumed kinetic temperature of $50 \mathrm{~K}$, while the lower two panels assume $T_{K}=100 \mathrm{~K}$. For a given transition ratio, the derived spatial density is quite sensitive to the assumed $T_{K}$. 


\subsection{Model Results}

\subsubsection{Derived Source Physical Parameters}

In the following sections we derive the best-fit LVG solution to the ensemble of observed $\mathrm{H}_{2} \mathrm{CO}$ radiation temperatures for each molecular cloud in our sample. This best-fit solution is found by minimizing $\chi^{2}$, defined as

$$
\chi^{2}=\sum \frac{\left[T_{R}(\text { observed })-T_{R}(\text { model })\right]^{2}}{\left[\sigma\left(T_{R}(\text { observed })\right)\right]^{2}},
$$

within a three-dimensional parameter space defined by $T_{K}$, $n\left(\mathrm{H}_{2}\right)$, and $N$ (species- $\left.\mathrm{H}_{2} \mathrm{CO}\right) / \Delta v$. The grid spacing in this three-dimensional parameter space was $5 \mathrm{~K}$ in $T_{K}$ and 0.05 in $\log \left[n\left(\mathrm{H}_{2}\right)\right]$ and $\log \left[N\left(\right.\right.$ species- $\left.\left.\mathrm{H}_{2} \mathrm{CO}\right) / \Delta v\right]$. The uncertainty in $T_{R}$ (observed $), \sigma\left(T_{R}\right.$ (observed $\left.)\right)$, includes the rms noise in the spectra and the absolute temperature calibration uncertainties. The statistical uncertainty for these fits is a function of the number of degrees of freedom available. For 1 degree of freedom, $1 \sigma, 2 \sigma$, and $3 \sigma$ correspond to $\chi^{2}$ values of $1.1,3.8$, and 5.4, respectively. For 2, 3, and 4 degrees of freedom the $\chi^{2}$ values are $2.4,6.0$, and $7.8 ; 3.6,7.8$, and 9.7 ; and $4.8,9.5$, and 11.5 , respectively. The best-fit model and observed $T_{R}$ values for each source are shown in Figure 16. Since the best-fit solution to the physical parameters of a molecular cloud often cover a range in spatial density, kinetic temperature, and species column density, for a few sources we also show representative contour plots of $\chi^{2}$ within this three-dimensional parameter space. For all sources we have used the distribution of $\chi^{2}$ within this three-dimensional parameter space to derive the statistical uncertainties of our physical parameter estimates. Table 5 lists the best-fit and $3 \sigma$ fit range for our estimates of $T_{K}, n\left(\mathrm{H}_{2}\right)$, and $N\left(\right.$ species $\left.-\mathrm{H}_{2} \mathrm{CO}\right) / \Delta v$ in each source. In the following sections we discuss our LVG model results within the context of the known properties of each source.

\subsubsection{W3 IRS 4}

W3 IRS 4 is the site of a dense molecular cloud core and embedded infrared source located near the core of the W3 star formation complex. Extensive studies of its infrared (WynnWilliams, Becklin, \& Neugebauer 1972; Werner et al. 1980), submillimeter (Gordon 1987; Richardson et al. 1989b), and molecular spectral line (Phillips et al. 1981; Wright, Dickel, \& Ho 1984; Thronson 1986; Hayashi, Kobayashi, \& Hasegawa 1989) emission have found that W3 IRS 4 is a dense $\left[n\left(\mathrm{H}_{2}\right) \simeq\right.$ $\left.10^{5}-10^{6} \mathrm{~cm}^{-3}\right]$, compact $\left(\theta_{s} \simeq 40^{\prime \prime}-60^{\prime \prime}\right)$, massive $(M \simeq 500-$ $\left.2000 M_{\odot}\right)$, and luminous $\left(L \simeq 10^{5} L_{\odot}\right)$ region of recent star formation. Kinetic temperature estimates from these studies range from 55 to $80 \mathrm{~K}$ for measurements using $15^{\prime \prime}-30$ " beams.

The results from our para- $\mathrm{H}_{2} \mathrm{CO} \mathrm{LVG}$ analysis are given in Table 5, and the best-fit model result is shown in Figure $16 a$. Good agreement is found between our direct $n\left(\mathrm{H}_{2}\right)$ and $T_{K}$ measurements and the estimates described above. In Figure 17 we show contour plots of $\chi^{2}$ from our LVG model solutions in $n\left(\mathrm{H}_{2}\right)-N\left(\right.$ para- $\left.\mathrm{H}_{2} \mathrm{CO}\right) / \Delta v-T_{K}$ parameter space. This is a good example of a weakly constrained ( 1 degree of freedom) fit and shows the interplay between the three physical parameters of the model. As indicated in Figure 17, poorly determined LVG solutions become unconstrained at the low $n\left(\mathrm{H}_{2}\right)$, high
$N\left(\right.$ para- $\left.\mathrm{H}_{2} \mathrm{CO}\right) / \Delta v$, high $T_{K}$ and high $n\left(\mathrm{H}_{2}\right)$, low $N$ (para$\left.\mathrm{H}_{2} \mathrm{CO}\right) / \Delta v$, low $T_{K}$ corners of parameter space.

\subsubsection{2. $\mathrm{W} 3(\mathrm{OH})$}

W3 $(\mathrm{OH})$ is a compact $\mathrm{H}$ II region and molecular cloud core located $\sim 18^{\prime}$ southeast of W3 IRS 4 . The 1 '.2 resolution HCN measurements of Turner \& Welch (1984) found that $\mathrm{W} 3(\mathrm{OH})$ is composed of two components: one [at $V_{\mathrm{LSR}} \simeq-47$ $\mathrm{km} \mathrm{s}^{-1}$, often referred to as $\left.\mathrm{W} 3(\mathrm{OH})\right]$ located at the position of the compact $\mathrm{H}$ II region, and a second [at $V_{\mathrm{LSR}} \simeq-52 \mathrm{~km}$ $\mathrm{s}^{-1}$, often referred to as $\left.\mathrm{W} 3\left(\mathrm{H}_{2} \mathrm{O}\right)\right]$ located $\sim 7^{\prime \prime}$ east near the position of several $\mathrm{H}_{2} \mathrm{O}$ maser emission centers (Dreher \& Welch 1981). The association with $\mathrm{H}_{2} \mathrm{O}$ maser emission, high $\mathrm{HCN}$ brightness temperature, and broad line width $(\Delta v \simeq 10$ $\left.\mathrm{km} \mathrm{s}^{-1}\right)$ lead Turner \& Welch to suggest that $\mathrm{W} 3\left(\mathrm{H}_{2} \mathrm{O}\right)$ contains an obscured young star or protostar. Wilson, Johnston, \& Mauersberger (1991) also find evidence for this two-component structure. Their measurements of the CS $J=3 \rightarrow 2, \mathrm{C}^{34} \mathrm{~S}$ $J=3 \rightarrow 2$, and $\mathrm{C}^{34} \mathrm{~S} J=5 \rightarrow 4$ emission toward this region indicate that near $\mathrm{W} 3(\mathrm{OH}) V_{\mathrm{LSR}}>-47 \mathrm{~km} \mathrm{~s}^{-1}$, while near $\mathrm{W} 3\left(\mathrm{H}_{2} \mathrm{O}\right) V_{\mathrm{LSR}}<-47 \mathrm{~km} \mathrm{~s}^{-1}$ with a broadening of the line widths as one moves from $\mathrm{W} 3(\mathrm{OH})$ to $\mathrm{W} 3\left(\mathrm{H}_{2} \mathrm{O}\right)$. These and other studies of the molecular spectral line emission (Reid, Myers, \& Bieging 1987; Mauersberger, Wilson, \& Henkel 1988 ) indicate that the average physical conditions in both $\mathrm{W} 3(\mathrm{OH})$ and $\mathrm{W} 3\left(\mathrm{H}_{2} \mathrm{O}\right)$ are $n\left(\mathrm{H}_{2}\right) \gtrsim 10^{5} \mathrm{~cm}^{-3}, T_{K} \gtrsim 50 \mathrm{~K}$, $M \simeq 100 M_{\odot}$ and $\theta_{s} \lesssim 15^{\prime \prime}$. Measurements of the 50 and 100 $\mu \mathrm{m}$ continuum emission (Campbell et al. 1989) indicate that $T_{K} \simeq 45 \mathrm{~K}$ within a 30 " beam.

The results from our para- $\mathrm{H}_{2} \mathrm{CO} \mathrm{LVG}$ analysis are given in Table 5, and the best-fit model result is shown in Figure $16 b$. Good agreement is found between our direct $n\left(\mathrm{H}_{2}\right)$ measurement and the estimates described above. Previous gas kinetic temperature estimates were based on measurements of low-excitation transitions. Therefore, our $\mathrm{H}_{2} \mathrm{CO}$ gas kinetic temperature measurements represent the first to detect warm $\left(T_{K} \sim\right.$ $110 \mathrm{~K})$ gas associated with $\mathrm{W} 3(\mathrm{OH})$.

\subsubsection{Orion-KL}

Orion-KL (OMC-1) is the best-studied region of massive star formation in our Galaxy (see reviews by Genzel \& Stutzki 1989 and Genzel 1991$)$. High-resolution $\left(\theta_{B} \lesssim 30^{\prime \prime}\right)$ studies of the molecular spectral line emission toward this region find that it is composed of three major components; the hot core $\left(V_{\mathrm{LSR}} \simeq 3-5 \mathrm{~km} \mathrm{~s}^{-1}, \Delta v \simeq 10-15 \mathrm{~km} \mathrm{~s}^{-1}, T_{K} \simeq 150-300 \mathrm{~K}\right)$, ridge $\left(V_{\mathrm{LSR}} \simeq 8-10 \mathrm{~km} \mathrm{~s}^{-1}, \Delta v \simeq 3-6 \mathrm{~km} \mathrm{~s}^{-1}, T_{K} \simeq 50-150 \mathrm{~K}\right)$, and plateau $\left(V_{\mathrm{LSR}} \simeq 7-8 \mathrm{~km} \mathrm{~s}^{-1}, \Delta v \gtrsim 20 \mathrm{~km} \mathrm{~s}^{-1}, T_{K} \simeq 100\right.$ $150 \mathrm{~K}$ ) (Blake et al. 1987; Mangum et al. 1990). The ridge component is often separated into "compact" $\left(\theta_{s} \leqslant 30\right.$ ") and "extended" $\left(\theta_{s}>30\right.$ ") subcomponents. All components have $n\left(\mathrm{H}_{2}\right) \gtrsim 10^{5} \mathrm{~cm}^{-3}$.

Several of our measured para- $\mathrm{H}_{2} \mathrm{CO}$ transitions appear to be contaminated by other spectral lines (see Table 3 and Figure $3 a$ ). The $5_{05} \rightarrow 4_{04}$ and $5_{23} \rightarrow 4_{22}$ line profiles are contaminated with unidentified lines, while the $5_{24} \rightarrow 4_{23}$ line profile is partially blended with $\mathrm{SO}_{2}$. Excluding the measurements contaminated with unidentified lines, we derive the kinetic temperatures, spatial densities, and para- $\mathrm{H}_{2} \mathrm{CO}$ column densities per unit line width listed in Table 5. Figures $16 c, 16 d$, and $16 e$ 
display the best fits to these measurements for each component. Our results for the hot core and ridge components are in agreement with previous measurements (Blake et al. 1987; Mangum et al. 1990; Mangum et al. 1993), while our fit to the kinetic temperature in the plateau component is approximately twice that derived from other measurements (Blake et al. 1987). The uncertainty associated with our plateau $T_{K}$ estimate is rather large, and the $T_{R}$ measurements used to derive this estimate are based upon three-Gaussian deconvolutions of the measured spectral profiles. Therefore, we cannot express a great deal of faith in our plateau $T_{K}$ derivation. Since the ortho$\mathrm{H}_{2} \mathrm{CO}$ transitions measured toward the Orion-KL components attain moderate opacities, we have not attempted to constrain ortho- $\mathrm{H}_{2} \mathrm{CO}$ LVG models of these components.

\subsubsection{OMC-2 IRS 4}

OMC-2 IRS 4 is a young stellar object located near the center of the OMC-2 component of the Orion A molecular cloud. OMC-2 IRS 4 is a double source at $2.2 \mu \mathrm{m}$ (Rayner et al. 1989; Johnson et al. 1990). The northern component of this double source is thought to be a pre-main-sequence star with a luminosity of $1500 L_{\odot}$ which is embedded in an east-westoriented protostellar disk (Rayner et al. 1989). OMC-2 IRS 4 possesses a north-south-directed $\mathrm{CO}$ outflow (Fischer et al. 1985) and a north-south bipolar reflection nebula (Fischer, Righini-Cohen, \& Simon 1980). It is also a source of $\mathrm{H}_{2} \mathrm{O}$ maser emission (Genzel \& Downes 1979). Batrla et al. (1983) measured the $\mathrm{NH}_{3}$ emission toward OMC-2 IRS 4 and found a source $\simeq 30^{\prime \prime}$ in size located $\sim 20^{\prime \prime}$ to the southwest of the 2.2 $\mu \mathrm{m}$ source position. For the $\mathrm{NH}_{3}$ core Batrla et al. derived $T_{K} \simeq$ $25 \mathrm{~K}$ and $n\left(\mathrm{H}_{2}\right) \simeq 10^{5} \mathrm{~cm}^{-3}$. More recently, Mezger, Wink, \& Zylka (1990) made high-resolution $\left(\theta_{B} \simeq 11^{\prime \prime}\right)$ measurements of the $1.3 \mathrm{~mm}$ dust continuum emission from this region. They found that the emission is dominated by a warm $\left(T_{K} \simeq\right.$ $40 \mathrm{~K})$, high-density $\left[n\left(\mathrm{H}_{2}\right) \simeq 10^{6} \mathrm{~cm}^{-3}\right]$ condensation of size $\sim 20^{\prime \prime}$ located near the $\mathrm{NH}_{3}$ emission region (Mezger, Wink, \& Zylka designate this continuum source FIR 4).

Our para- $\mathrm{H}_{2} \mathrm{CO}$ measurements of OMC-2 lRS 4 were done at the $2.2 \mu \mathrm{m}$ source position. Derivations of the kinetic temperature, spatial density, and para- $\mathrm{H}_{2} \mathrm{CO}$ column density per unit line width are listed in Table 5. In Figure $16 f$ we show our best-fit LVG model to these measurements. Even though we have 4 degrees of freedom for this fit, the best-fit solution is at the $\sim 2 \sigma$ level. The $3 \sigma$ fit range, though, is well constrained and indicates $T_{K} \gtrsim 75 \mathrm{~K}, 2-3$ times higher than previously measured.

\subsubsection{NGC 2024}

NGC 2024 (Orion B) is a well-studied, nearby star-forming region. High-resolution $\left(\theta_{B} \lesssim 30^{\prime \prime}\right)$ measurements of the dust continuum (Mezger et al. 1992) and molecular spectral line emission (Schulz et al. 1991; Gaume, Johnston, \& Wilson 1992; Mauersberger et al. 1992) have detected a string of seven dense $\left[n\left(\mathrm{H}_{2}\right) \sim 10^{5}-10^{8} \mathrm{~cm}^{-3}\right]$ cores embedded within a north-south-extended $0.3 \times 0.5 \mathrm{pc}$ ridge of molecular material. The kinetic temperatures of the cores located near the center of the ridge (designated FIR 4 and FIR 5 by Mezger et al. 1990), just south of the NGC $2024 \mathrm{H}$ II region, are found to be much higher $\left(T_{K} \gtrsim 40 \mathrm{~K}\right.$; Mauersberger et al. 1992) than the cores located in the northern and southern portions of the ridge $\left(T_{K} \sim 20 \mathrm{~K}\right.$; Mezger et al. 1992; Mauersberger et al. 1992). Mezger et al. (1988, 1992) have suggested that the dense cores in NGC 2024 are protostellar objects.

We have obtained measurements of several para- and ortho$\mathrm{H}_{2} \mathrm{CO}$ transitions toward the central position in the NGC 2024 ridge (approximately midway between FIR 4 and FIR 5 ). Our derivations of $T_{K}, n\left(\mathrm{H}_{2}\right)$, and $N\left(\right.$ para- $\left.\mathrm{H}_{2} \mathrm{CO}\right) / \Delta v$ based on these measurements are given in Table 5 , while the model predictions for the best-fit solution are displayed in Figure $16 \mathrm{~g}$. The value of $n\left(\mathrm{H}_{2}\right)$ derived from our para- $\mathrm{H}_{2} \mathrm{CO}$ measurements is in good agreement with estimates based on previous measurements. Our derived $T_{K}$ of $95 \mathrm{~K}$, however, is much larger than previously measured. Moreover, as a check on our para- $\mathrm{H}_{2} \mathrm{CO} T_{K}$ measurements, we note that our measured $T_{R}\left(5_{15} \rightarrow 4_{14}\right) / T_{R}\left(5_{33} \rightarrow 4_{32}\right)$ ratio of $6.48 \pm 1.51$ implies that $T_{K}=95_{-35}^{+10} \mathrm{~K}$, in good agreement with our para- $\mathrm{H}_{2} \mathrm{CO}$ measurements. This discrepancy between the current $T_{K}$ measurements and those made previously probably arises from the fact that previous estimates of $T_{K}$ were based on lower excitation tracers, such as transitions between low-lying molecular energy levels in linear molecules and emission from cool dust. With the resolution achieved in our $\mathrm{H}_{2} \mathrm{CO}$ measurements, we cannot be certain where the warm $\mathrm{H}_{2} \mathrm{CO}$ is located; it may closely associate with the dust clumps, or it may lie near the edge of the $\mathrm{H}$ II region. Mezger et al. (1992) have maintained that the condensations derive all of their energy from free-fall contraction of isothermal protostars, while Moore \& Chandler (1989) postulate that FIR 4 contains an embedded source with luminosity of a few hundred solar luminosities. We suggest that determination of the source of the high-excitation $\mathrm{H}_{2} \mathrm{CO}$ emission could resolve this dispute.

\subsubsection{NGC 2071 IRS 1}

NGC 2071 is a visible reflection nebula located within the L1630 (Orion B) molecular cloud. An area containing compact $\mathrm{H}$ II regions (Snell \& Bally 1986), OH (Johansson et al. 1974), and $\mathrm{H}_{2} \mathrm{O}$ (Genzel \& Downes 1979) maser emission, a bipolar molecular outflow seen in CO (Lichten 1982; Bally 1982; Snell et al. 1984; Moriarty-Schieven, Snell, \& Hughes 1989), $\mathrm{H}_{2}$ (Persson et al. 1981; Lane \& Bally 1986), $\mathrm{HCO}^{+}$ (Wootten et al. 1984), and CS (Takano et al. 1984; Mead et al. 1989), and embedded infrared emission sources (Evans et al. 1979; Harvey et al. 1979) is located $\sim 3^{\prime}$ north of this reflection nebula.

Dust continuum emission measurements at 50 and $100 \mu \mathrm{m}$ (Butner et al. 1990) and multitransition $\mathrm{NH}_{3}$ (Zhou, Evans, \& Mundy 1990) and CS (Zhou et al. 1991) emission studies of this region have produced detailed models of the source structure in both spatial density and dust temperature. Using a spherically symmetric radiative transport code, Butner et al. find that the density distribution can be described by a power law, centered on IRS 1 , of the form $n(r)=n_{i}\left(r / r_{i}\right)^{-\alpha}$ with $n_{i}=$ $(0.7-8.0) \times 10^{6} \mathrm{~cm}^{-3}, \alpha=1.5-2.0$, and $r_{i}=5^{\prime \prime}$. The self-consistent dust temperature distribution derived from this model is somewhat dependent upon the assumed dust properties, but yields $T_{d} \simeq 25 \mathrm{~K}$ for $\theta_{s} \simeq 30^{\prime \prime}$, which increases to $\simeq 80 \mathrm{~K}$ for $\theta_{s} \simeq$ 10 ", with a central source luminosity of $520 L_{\odot}$. The molecular gas distribution shows an interesting morphology, with the 

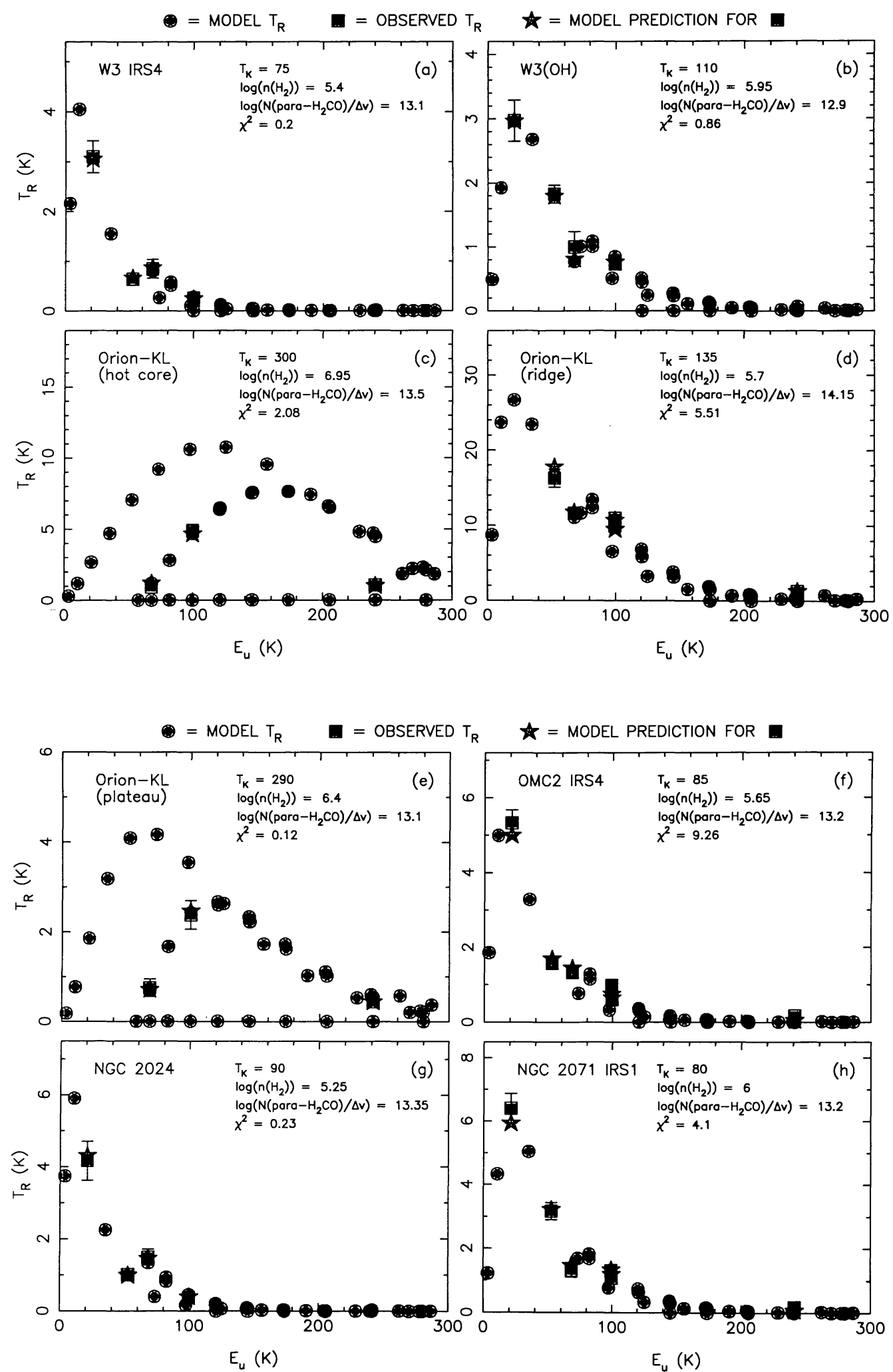

FIG. 16.-Comparison between the best-fit LVG model-predicted and measured $\mathrm{H}_{2} \mathrm{CO}$ radiation temperatures for all of the sources in our sample. The physical parameters associated with the best-fit LVG solution are shown for each source. All species- $\mathrm{H}_{2} \mathrm{CO}$ transitions are represented; reference to $E_{u}$ in Fig. 12 can be used to identify individual transitions. 

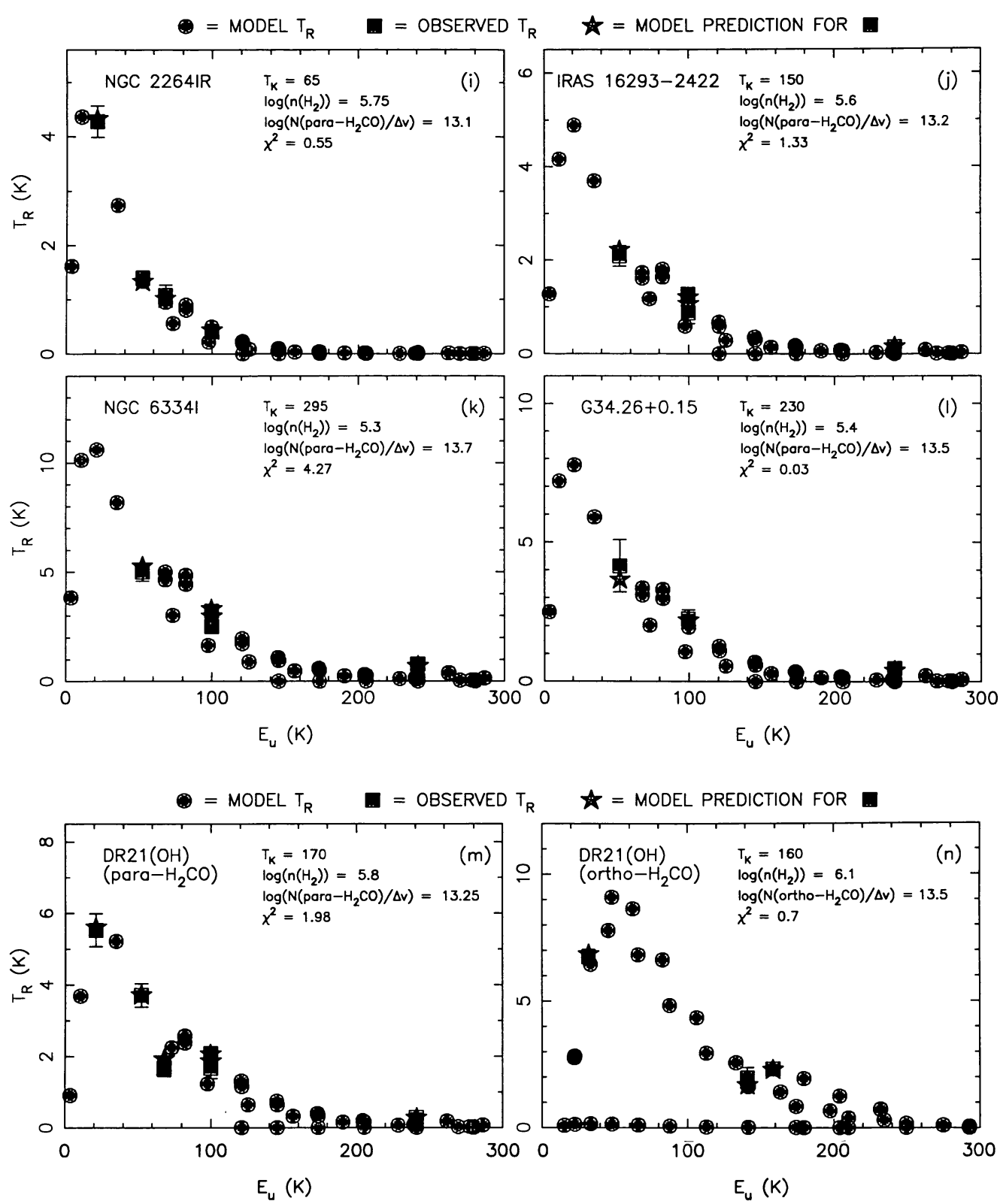

FIG. 16-Continued

$\mathrm{NH}_{3}$ emission forming a ringlike structure around the dense core defined by the CS emission which is centered on IRS 1 . Using a radiative transport model which is similar to that used by Butner et al., Zhou et al. (1991) find that the dense gas traced by the CS emission can be described by a power law with $n_{i}=3 \times 10^{6} \mathrm{~cm}^{-3}, \alpha=1.3$, and $r_{i}=5^{\prime \prime}$, similar to that derived from the dust distribution.

Our para- $\mathrm{H}_{2} \mathrm{CO}$ LVG model of NGC 2071 has 4 degrees of freedom, making it one of the most well-constrained models in our set. For this reason we display in Figure 18 contour diagrams of the goodness-of-fit parameter $\chi^{2}$ as a function of $n\left(\mathrm{H}_{2}\right), N\left(\right.$ para- $\left.\mathrm{H}_{2} \mathrm{CO}\right) / \Delta v$, and $T_{K}$. Figure 18 should be compared with the $\chi^{2}$ diagram for W3 IRS 4 (Fig. 17) to see the difference between a well-constrained and a poorly constrained model. Our derivations of $T_{K}, n\left(\mathrm{H}_{2}\right)$, and $N$ (para$\left.\mathrm{H}_{2} \mathrm{CO}\right) / \Delta v$ are given in Table 5, while the para- $\mathrm{H}_{2} \mathrm{CO}$ radia- tion temperature model predictions for the best-fit solution are displayed in Figure 16h. In comparison with the spatial density distribution derived from the CS emission model of Zhou et al. (1991) and the spatial density and dust temperature distribution derived from the far-infrared dust emission model of Butner et al. (1990), our derived $n\left(\mathbf{H}_{2}\right)$ would be appropriate for $\theta_{s}=7 "-28^{\prime \prime}$, and our derived $T_{K}$ would be appropriate for $\theta_{s} \simeq 10^{\prime \prime}$ (assuming $T_{d}=T_{K}$ ).

Mundy, Mangum, \& Salter (1993) have used maps of the $\mathrm{H}_{2} \mathrm{CO}_{03} \rightarrow 2_{02}, 3_{22} \rightarrow 2_{21}$, and $5_{05} \rightarrow 4_{04}$ transitions along with measurements of the central intensities of the $5_{24} \rightarrow 4_{23}$ and $5_{42} \rightarrow 4_{41} / 5_{41} \rightarrow 4_{40}$ transitions to constrain nonuniform microturbulent radiative transfer models of the $\mathrm{H}_{2} \mathrm{CO}$ emission toward NGC 2071 IRS 1. For the central position (which is coincident with our observed position), Mundy et al. (1993) derive a central $T_{K}$ and $n\left(\mathrm{H}_{2}\right)$ similar to our measured values. 
TABLE 5

$\mathrm{H}_{2}$ CO MODEL Results

\begin{tabular}{|c|c|c|c|c|c|c|c|c|c|c|}
\hline \multirow[b]{2}{*}{ SOURCE } & \multirow[b]{2}{*}{$\mathrm{DOF}^{\mathrm{a}}$} & \multicolumn{5}{|c|}{ BEST FIt } & \multicolumn{4}{|c|}{$3 \sigma$ FIt RANGE } \\
\hline & & $\begin{array}{r}T_{K} \\
(\mathrm{~K})\end{array}$ & $\begin{array}{c}\log \left[n\left(\mathrm{H}_{2}\right)\right] \\
\left(\mathrm{cm}^{-3}\right)\end{array}$ & & $\begin{array}{l}{\left[\frac{N\left(\text { para- } \mathrm{H}_{2} \mathrm{CO}\right)}{\Delta v}\right]^{\mathrm{b}}} \\
\left.\mathrm{m}^{-2}\left(\mathrm{~km} \mathrm{~s}^{-1}\right)^{-1}\right]\end{array}$ & $\chi^{2}$ & $\begin{array}{c}T_{K} \\
(\mathrm{~K})\end{array}$ & $\begin{array}{c}\log \left[n\left(\mathrm{H}_{2}\right)\right] \\
\left(\mathrm{cm}^{-3}\right)\end{array}$ & & $\begin{array}{l}{\left[\frac{N\left(\text { para- } \mathrm{H}_{2} \mathrm{CO}\right)}{\Delta v}\right]^{\mathrm{b}}} \\
\left.\mathrm{cm}^{-2}\left(\mathrm{~km} \mathrm{~s}^{-1}\right)^{-1}\right]\end{array}$ \\
\hline W3IRS4 & 1 & $75_{-15}^{+20}$ & $5.40_{-0.20}^{+0.10}$ & & $13.10 \pm 0.10$ & 0.20 & $45-190$ & $4.60-5.75$ & & $12.90-13.70$ \\
\hline $\mathrm{w} 3(\mathrm{OH}) \ldots \ldots \ldots \ldots \ldots$ & 1 & $110_{-15}^{+5}$ & $5.95_{-0.01}^{+0.05}$ & & $12.90 \pm 0.01$ & 0.86 & $70-200$ & $5.60-6.25$ & & $12.82-13.00$ \\
\hline \multicolumn{11}{|l|}{ Orion-KL: } \\
\hline Hot core $^{c} \ldots \ldots \ldots$ & 1 & 300 & 6.95 & & 13.50 & $2.08^{d}$ & $225->300$ & $6.55->7.50$ & & $13.40-13.60$ \\
\hline Ridge $\ldots \ldots \ldots \ldots \ldots$ & 3 & 135 & 5.70 & & 14.15 & $5.51^{\mathrm{d}}$ & $120-160$ & $5.55-5.77$ & & $14.10-14.20$ \\
\hline Plateau $^{\mathrm{c}} \ldots \ldots \ldots \ldots$ & 1 & $290_{-135}^{+>10}$ & $6.40_{-0.40}^{+>1.20}$ & & $13.10_{-0.06}^{+>0.18}$ & $0.12^{\mathrm{e}}$ & $125->300$ & $5.90->7.60$ & & $12.97->13.35$ \\
\hline OMC-2 IRS $4 \ldots \ldots \ldots$ & 4 & 85 & 5.65 & & 13.20 & $9.26^{\mathrm{f}}$ & $75-110$ & $5.50-5.72$ & & $13.15-13.25$ \\
\hline NGC $2024 \ldots \ldots \ldots \ldots$ & 1 & $95_{-20}^{+30}$ & $5.25 \pm 0.15$ & & $13.35 \pm 0.10$ & 0.23 & $60-270$ & $<4.20-5.65$ & & $13.10->14.30$ \\
\hline NGC 2071 IRS $1 \ldots \ldots$ & 4 & $80_{-15}^{+20}$ & $6.00_{-0.15}^{+0.10}$ & & $13.20_{-0.03}^{+0.05}$ & 4.10 & $50-125$ & $5.73-6.30$ & & $13.12-13.31$ \\
\hline NGC 2264 IR .......... & 1 & $65_{-0}^{+10}$ & $5.75 \pm 0.05$ & & $13.10_{-0.05}^{+0.03}$ & 0.55 & $45-105$ & $5.50-6.06$ & & $12.98-13.22$ \\
\hline IRAS $16293-2422 \ldots \ldots$ & 2 & $150_{-20}^{+85}$ & $5.60_{-1.00}^{+0.25}$ & & $13.20_{-0.30}^{+0.80}$ & 1.33 & $85->300$ & $<4.50->6.50$ & & $12.70->14.30$ \\
\hline NGC 6334I ........... & 2 & 295 & 5.30 & & 13.70 & $4.27^{\mathrm{d}}$ & $240->300$ & $4.50-5.50$ & & $13.50-14.50$ \\
\hline G34.26+0.15 $\ldots \ldots \ldots$ & 1 & 230 & 5.40 & & 13.50 & $0.03^{\mathrm{e}}$ & $85->300$ & $<4.5->7.0$ & & $13.00->14.50$ \\
\hline \multicolumn{11}{|l|}{ DR 21(OH): } \\
\hline Para- $\mathrm{H}_{2} \mathrm{CO} \ldots \ldots \ldots$ & 4 & $170_{-35}^{+50}$ & $5.80_{-0.10}^{+0.20}$ & & $13.25_{-0.07}^{+0.01}$ & 1.98 & $110-275$ & $5.60-6.10$ & & $13.14-13.31$ \\
\hline Ortho- $\mathrm{H}_{2} \mathrm{CO} \ldots \ldots \ldots$ & 2 & $160_{-40}^{+140}$ & $6.10_{-0.40}^{+>2.40}$ & & $13.50_{-0.02}^{+0.05}$ & 0.70 & $<70->300$ & $5.50->8.50$ & & $13.44-13.74$ \\
\hline
\end{tabular}

${ }^{a}$ Number of degrees of freedom.

b Note that for the DR $21(\mathrm{OH})$ ortho- $\mathrm{H}_{2} \mathrm{CO}$ model, the tabulated quantity is $\log \left[N\left(\right.\right.$ ortho- $\left.\left.\mathrm{H}_{2} \mathrm{CO}\right) / \Delta v\right]$.

${ }^{c}$ Note that the $5_{05} \rightarrow 4_{04}$ and $5_{23} \rightarrow 4_{22}$ transitions have been excluded from this analysis because of probable $u$-line confusion.

d Solution is at $\sim 1.5 \sigma$.

e Even though the model reaches a very small value of $\chi^{2}$, it is unconstrained over its $1 \sigma$ fit range.

${ }^{f}$ Solution is at $\sim 2.0 \sigma$.

The kinetic temperature and spatial density structure derived from the Mundy et al. (1993) measurements is also similar to that derived by Butner et al. (1990) and Zhou et al. (1991).

\subsubsection{NGC 2264IR}

First detected at $2.2 \mu \mathrm{m}$ by Allen (1972), NGC 2264IR is in a molecular cloud complex associated with the open cluster NGC 2264. NGC 2264IR is a source of $\mathrm{H}_{2} \mathrm{O}$ maser emission (Genzel \& Downes 1977) but has no known bipolar outflow (it is located $\sim 5^{\prime}$ south of the CO bipolar outflow source NGC 2264D discovered by Margulis, Lada, \& Snell 1988). Harvey, Campbell, \& Hoffman (1977) measured the 50, 100, and 175 $\mu \mathrm{m}$ emission from NGC 2264IR and found it to be $\$ 20^{\prime \prime}$ in size with a dust temperature of $\sim 35 \mathrm{~K}$. By analyzing the available photometry from 2.2 to $1000 \mu \mathrm{m}$, they estimated a luminosity of $\sim 3500 L_{\odot}$. Walker, Adams, \& Lada (1990) have mapped the $1.3 \mathrm{~mm}$ continuum emission toward NGC 2264IR. By modeling the 30-3350 $\mu \mathrm{m}$ continuum emission toward this source, they find that $T_{d} \simeq 40 \mathrm{~K}$ and $n\left(\mathrm{H}_{2}\right) \simeq 7 \times$ $10^{5} \mathrm{~cm}^{-3}$.

While all of our measured $\mathrm{H}_{2} \mathrm{CO}$ transitions show central velocities of between 8 and $9 \mathrm{~km} \mathrm{~s}^{-1}$, the $5_{05} \rightarrow 4_{04}$ and $5_{23} \rightarrow$ $4_{22}$ transitions possess a second velocity component at $\sim 4 \mathrm{~km}$ $\mathrm{s}^{-1}$ ( see Table 3 ). Although this $4 \mathrm{~km} \mathrm{~s}^{-1}$ component may be an artifact from the unstable AOS ( see $\S 2.1$ ), it could also be a second component with $T_{K} \gtrsim 60 \mathrm{~K}$. Table 5 lists our derivations of $T_{K}, n\left(\mathrm{H}_{2}\right)$, and $N\left(\right.$ para- $\left.\mathrm{H}_{2} \mathrm{CO}\right) / \Delta v$ based on our para-
$\mathrm{H}_{2} \mathrm{CO}$ measurements of the primary (8-9 $\mathrm{km} \mathrm{s}^{-1}$ ) velocity component in NGC 2264IR. The para- $\mathrm{H}_{2} \mathrm{CO}$ model radiation temperatures for the best-fit LVG model are shown in Figure $16 i$. Our derived $n\left(\mathrm{H}_{2}\right)$ is comparable to that derived by Walker et al. (1990), while our derived $T_{K}$ is somewhat larger than the dust temperatures derived by Walker et al. (1990) and Harvey et al. ( 1977). Given the uncertainties in both temperature estimates, the agreement between $T_{d}$ and our derived $T_{K}$ is satisfactory.

\subsubsection{IRAS $16293-2422$}

IRAS $16293-2422$ is a deeply embedded young stellar object located within the $\rho$ Oph molecular cloud complex. Numerous high-resolution millimeter- and centimeter-wavelength measurements of the molecular spectral line and continuum emission toward this region have uncovered a considerable amount of structure in this object. Cloud models which include the presence of infall (Walker et al. 1986), outflow (Wilking \& Claussen 1987; Walker et al. 1988; Wootten 1989), and rotational (Menten et al. 1987) motion have all been used to explain the complex structure of IRAS 16293-2422. By analyzing high-resolution interferometric measurements of the millimeter-wavelength dust continuum, $\mathrm{C}^{18} \mathrm{O}, \mathrm{CO}$, and $\mathrm{NH}_{3}$ emission, Mundy, Wootten, \& Wilking (1990) have developed a comprehensive model for the IRAS 16293-2422 region. In this model IRAS 16293-2422 is composed of a "static core" $\left[T_{K} \simeq 15 \mathrm{~K}, n\left(\mathrm{H}_{2}\right) \simeq 10^{4} \mathrm{~cm}^{-3}, \theta_{s} \simeq\right.$ 


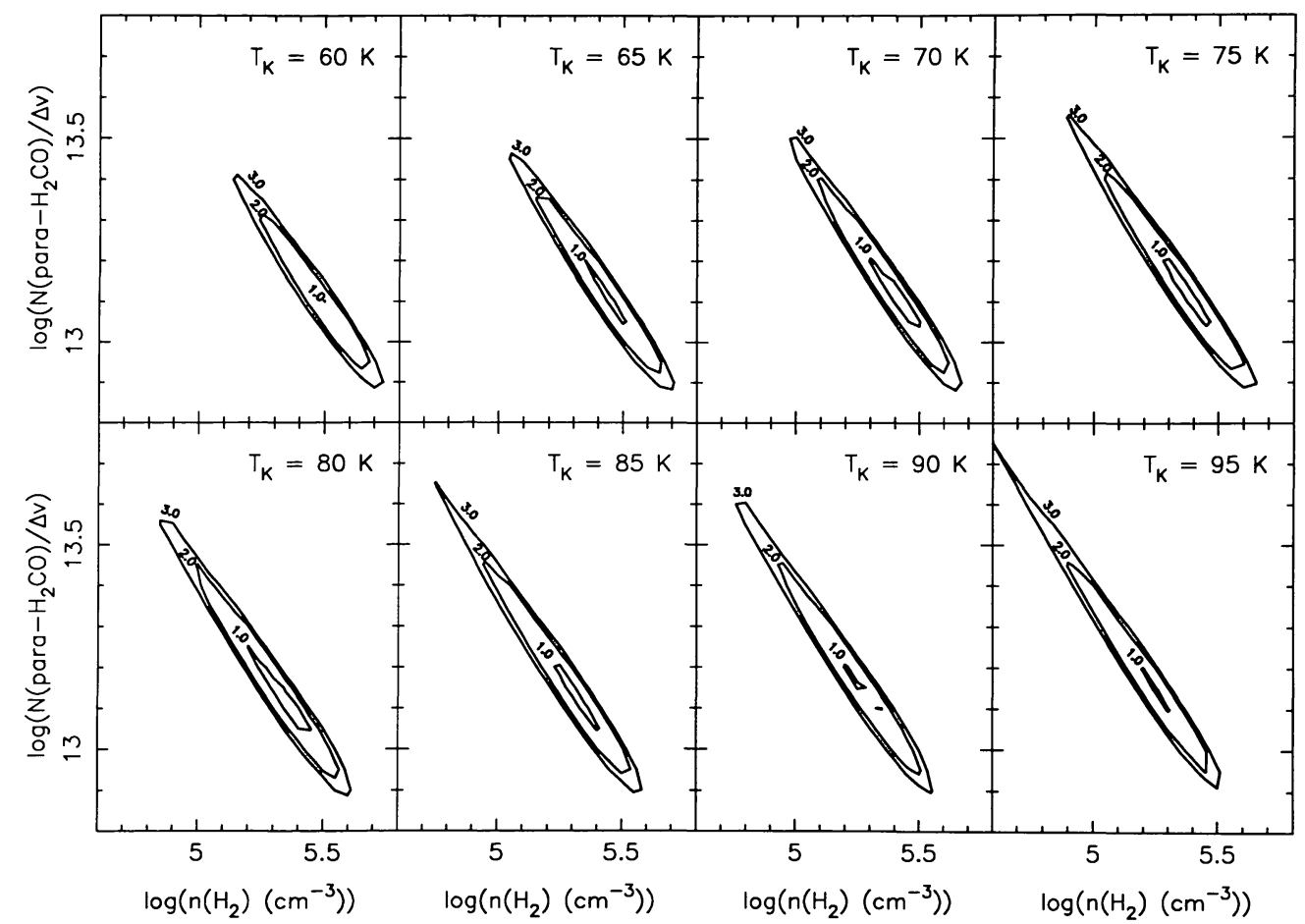

FIG. 17.-Contours of the goodness-of-fit parameter $\chi^{2}$ for the para- $\mathrm{H}_{2} \mathrm{CO}$ LVG model solutions of W3 IRS 4 . This is an example of a weakly constrained (1 degree of freedom) model fit.

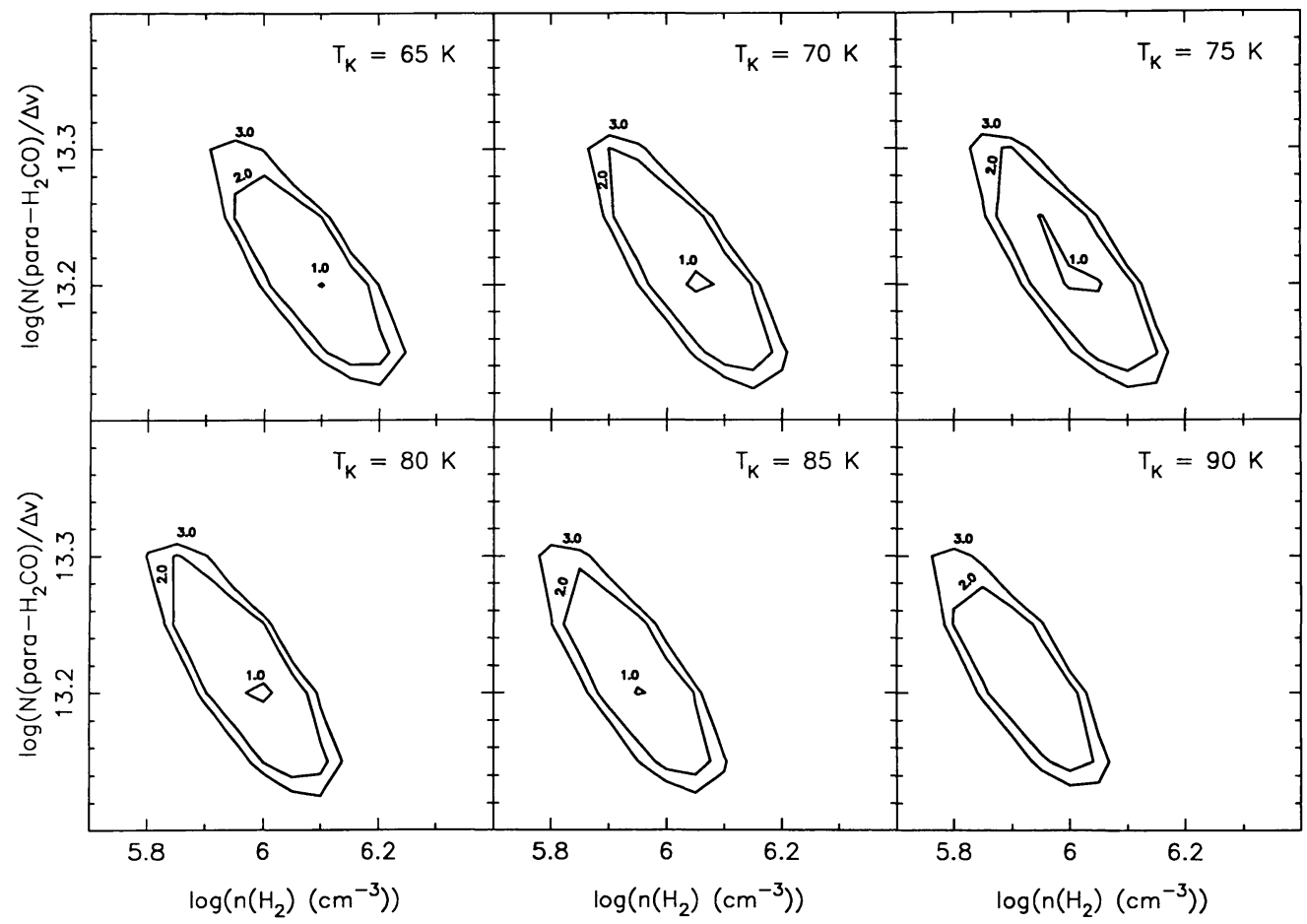

FIG. 18.-Contours of the goodness-of-fit parameter $\chi^{2}$ for the para- $\mathrm{H}_{2} \mathrm{CO}$ LVG model solutions of NGC 2071 IRS 1 . This is an example of a well-constrained ( 4 degrees of freedom) model fit. 
1'], an "outer disk" $\left.T_{K} \simeq 15 \mathrm{~K}, n\left(\mathrm{H}_{2}\right) \simeq 10^{5} \mathrm{~cm}^{-3}, \theta_{s} \simeq 30^{\prime \prime}\right]$, and a "disk" $\left[T_{K} \simeq 40 \mathrm{~K}, n\left(\mathrm{H}_{2}\right) \simeq 5 \times 10^{7} \mathrm{~cm}^{-3}, \theta_{s} \simeq 10^{\prime \prime}\right]$ component.

Table 5 lists our derivations of $T_{K}, n\left(\mathrm{H}_{2}\right)$, and $N$ (para$\left.\mathrm{H}_{2} \mathrm{CO}\right) / \Delta v$ based on our para- $\mathrm{H}_{2} \mathrm{CO}$ measurements of IRAS 16293-2422. LVG model results from the best-fit model are shown in Figure $16 j$. The most remarkable result from this model is our best-fit kinetic temperature of $150 \mathrm{~K}$. Even considering our derived $3 \sigma$ lower limit of $85 \mathrm{~K}$ (see Table 5), the kinetic temperature derived from our para- $\mathrm{H}_{2} \mathrm{CO}$ observations is greater than twice that estimated from previous measurements. As seems to be the case for many of the sources in our sample, previous estimates of the molecular gas kinetic temperature in IRAS 16293-2422 are based on low-excitation molecular transitions or cool dust, in this case $\mathrm{NH}_{3}$ and millimeter/ far-infrared continuum emission. However, on scales less than an arcsecond or so, water masers are seen arrayed around component "A," as is free-free emission (Wootten 1989). Emission around "B" at centimeter wavelengths may arise from free-free emission and from warm dust. The gas associated with any of these high-temperature components lies within 0.5 of either " $A$ " or " $B$ " and probably reflects kinetic temperatures of several hundred degrees. The high-excitation $\mathrm{H}_{2} \mathrm{CO}$ emission arises in a region whose temperature lies between these extremes, presumably on intermediate scales. Therefore, since the para- $\mathrm{H}_{2} \mathrm{CO}$ measurements presented in this work are sensitive to both the warm $\left(T_{K} \gtrsim 50 \mathrm{~K}\right)$ and $\operatorname{cool}\left(T_{K} \lesssim 50 \mathrm{~K}\right)$ gas in molecular cloud cores, our detection of warm $\left(T_{K}>85\right.$ $\mathrm{K}$ ) gas toward IRAS 16293-2422 suggests the presence of a component, probably closely associated with the star, which is not incorporated into current models of the IRAS 16293-2422 region.

\subsubsection{NGC 6334I}

The NGC 6334 molecular cloud complex is an extensive $(\theta \gtrsim 0.5)$ region of massive star formation. Numerous near-infrared (Straw, Hyland, \& McGregor 1989; Straw \& Hyland 1989) and far-infrared (McBreen et al. 1979; Harvey \& Gatley 1983; Loughran et al. 1986) sources are detected in this region, the strongest of which is NGC 6334I. Harvey \& Gatley (1983) and Loughran et al. (1986) resolved NGC 6334I into three components, IRS 1 , IRS 2, and IRS 3, of which IRS 1 is the brightest. From these far-infrared measurements NGC 6334I is found to have a compact core of size $\sim 8^{\prime \prime}$, a bolometric luminosity of $\sim 1.6 \times 10^{5} L_{\odot}$ and a dust temperature of $\sim 40$ K. IRS 1 also has associated compact H II (Rodriguez, Cantó, \& Moran 1982; Gaume \& Mutel 1987), OH maser (Gaume \& Mutel 1987), and $\mathrm{CH}_{3} \mathrm{OH}$ maser (Menten \& Batrla 1989) emission. Jackson, Ho, \& Haschick (1988) made high-resolution $\mathrm{NH}_{3}(1,1)$ emission measurements of NGC 6334I and found velocity structure which they attributed to a rotating circumstellar molecular disk. Measurements of the $\mathrm{CO}$ and $\mathrm{HC}_{3} \mathrm{~N}$ emission eminating from a massive bipolar outflow toward this object (Bachiller \& Cernicharo 1990) are inconsistent with the Jackson, Ho, \& Haschick interpretation. Bachiller \& Cernicharo suggest that the $\mathrm{NH}_{3}$ emission is probably tracing the high-density component of the bipolar outflow seen in $\mathrm{CO}$ and $\mathrm{HC}_{3} \mathrm{~N}$.

Derivations of $T_{K}, n\left(\mathrm{H}_{2}\right)$, and $N\left(\right.$ para- $\left.\mathrm{H}_{2} \mathrm{CO}\right) / \Delta v$ based on our para- $\mathrm{H}_{2} \mathrm{CO}$ measurements of NGC 6334I are listed in Ta- ble 5. LVG model results from the best-fit model are shown in Figure $16 \mathrm{k}$. Even though our model fit reaches only $1.5 \sigma$, our derived $3 \sigma$ lower limit of $T_{K}=240 \mathrm{~K}$ is approximately 6 times larger than the $40 \mathrm{~K}$ dust temperatures derived from the dust continuum measurements. This high kinetic temperature is apparent considering the high intensity of the $5_{42} \rightarrow 4_{41} / 5_{41} \rightarrow$ $4_{40}$ transition pair toward NGC 6334I, second only to that of Orion-KL (see Table 3 ). Only the compact ridge component of Orion-KL has a higher para- $\mathrm{H}_{2} \mathrm{CO}$ column density than NGC 6334I (see Table 5). NGC 6334I is also remarkable for its high spectral density of molecular lines. Transitions from $\mathrm{CH}_{3} \mathrm{OH}, \mathrm{HNC}$, and several unidentified species (one of which is shown in the $5_{33} \rightarrow 4_{32} / 5_{32} \rightarrow 4_{31}$ spectrum of Fig. 9) appear in our spectra. Therefore, NGC 6334I appears to be a hot, massive star-forming region.

\subsubsection{G34.26+0.15}

Located $\sim 40^{\prime}$ from the W44 supernova remnant, G34.26+0.15 is a cometary ultracompact H II region ( Reid \& Ho 1985) embedded within a dense molecular cloud. Measurements of the $\mathrm{CH}_{3} \mathrm{CN}$ emission toward this region (Churchwell, Walmsley, \& Wood 1992) indicate that it has a rather high kinetic temperature $\left(T_{K} \simeq 150 \mathrm{~K}\right)$. Measurements of the $C^{34} \mathrm{~S}$ emission indicate that $n\left(\mathrm{H}_{2}\right) \simeq 5 \times 10^{5} \mathrm{~cm}^{-3}$ and $\theta_{s} \simeq 30^{\prime \prime}$ (Cesaroni et al. 1991). Our derivations of $n\left(\mathrm{H}_{2}\right)$ and $T_{K}$ based on our para- $\mathrm{H}_{2} \mathrm{CO}$ measurements (Tables 5 and 3 and Figure $16 l)$ are consistent with the previous results listed above. Unfortunately, our LVG models are poorly constrained.

$$
\text { 4.5.1.11. DR 21( } \mathrm{OH})
$$

Located $\sim 3$ ' north of the massive O-star nursery DR 21 , DR $21(\mathrm{OH})$ is a star formation region in an early state of evolution. DR $21(\mathrm{OH})$ is composed of three compact emission regions, each of which appear to harbor a cluster of B stars (Woody et al. 1989; Mangum, Wootten, \& Mundy 1991). High-resolution measurements of the $\mathrm{NH}_{3}(1,1)$ and $\mathrm{NH}_{3}(2$, 2) emission toward this region (Mangum, Wootten, \& Mundy 1992) discovered a very clumpy medium with component kinetic temperatures in the range from 20 to higher than $80 \mathrm{~K}$. The $\mathrm{NH}_{3}$ emission over a $2^{\prime}$ region in these measurements appears to form a shell-like structure, suggesting an interaction between the dense gas and the young forming stars in this region.

The current $\mathrm{H}_{2} \mathrm{CO}$ measurements were obtained toward the DR $21(\mathrm{OH})$ main position in DR $21(\mathrm{OH})$. DR $21(\mathrm{OH})$ main contains two major components; MM 1 and MM 2. MM 1 has associated $\mathrm{OH} / \mathrm{H}_{2} \mathrm{O} / \mathrm{CH}_{3} \mathrm{OH}$ maser emission (Norris et al. 1982; Plambeck \& Menten 1990; Mangum et al. 1992) and is a strong source of far-infrared (Harvey et al. 1986), submillimeter (Gear et al. 1988; Richardson, Sandell, \& Krisciunas 1989a), and millimeter (Woody et al. 1989; Mangum et al. 1991 ) continuum emission. Mangum et al. (1991, 1992) found that MM 1 is a moderately luminous source $\left(L \simeq 10^{4}\right.$ $L_{\odot}$ ) which has $T_{K} \gtrsim 80 \mathrm{~K}$ over a source size of $\$ 10^{\prime \prime}$ at $V_{\mathrm{LSR}} \simeq$ $-4.1 \mathrm{~km} \mathrm{~s}^{-1}$. The MM 2 component of DR $21(\mathrm{OH})$ main is a somewhat less luminous $\left(L \simeq 10^{3} L_{\odot}\right)$ core which is spatially (located $\sim 8^{\prime \prime}$ southwest) and kinematically $\left(V_{\mathrm{LSR}} \simeq-0.7 \mathrm{~km}\right.$ $\mathrm{s}^{-1}$ ) distinct from MM 1. MM 2, like MM 1, has associated $\mathrm{H}_{2} \mathrm{O}$ maser emission (Mangum et al. 1992). The source size 
for MM 2 is similar to that of MM $1\left(\$ 10^{\prime \prime}\right)$, but it is a much cooler $\left(T_{K} \simeq 30 \mathrm{~K}\right)$ object. The lowest excitation transition $\left(3_{03} \rightarrow 2_{02}\right.$ at $\left.E_{u}=21 \mathrm{~K}\right)$ in our analysis shows a velocity component at $-1.0 \mathrm{~km} \mathrm{~s}^{-1}$ (associated with MM 2), while the higher excitation $\left(E_{u}>40 \mathrm{~K}\right)$ transitions are dominated by the MM 1 component at $\sim-3.0$ to $-4.0 \mathrm{~km} \mathrm{~s}^{-1}$. For the MM 2 component, we measure a lower limit of 3.7 to the ratio $T_{R}\left(3_{03} \rightarrow 2_{02}\right) / T_{R}\left(3_{22} \rightarrow 2_{21}\right)$, which indicates that $T_{K} \lesssim 100$ $\mathrm{K}$, consistent with previous measurements (Mangum et al. 1991, 1992).

Since DR $21(\mathrm{OH})$ is the source for which we have the most $\mathrm{H}_{2} \mathrm{CO}$ data ( 7 para- and 5 ortho- $\mathrm{H}_{2} \mathrm{CO}$ transitions), we have been able to constrain two separate LVG models for this source. Table 5 lists the results from our para- and ortho- $\mathrm{H}_{2} \mathrm{CO}$ models of DR $21(\mathrm{OH})$. Our derivations of the kinetic temperature and spatial density from the two $\mathrm{H}_{2} \mathrm{CO}$ species are in excellent agreement. The best-fit LVG solution to the ortho$\mathrm{H}_{2} \mathrm{CO}$ transitions is slightly more uncertain than that for the para- $\mathrm{H}_{2} \mathrm{CO}$ measurements, primarily because of the larger optical depth in the ortho transitions. Our derived kinetic temperature of $\sim 160 \mathrm{~K}$ and spatial density of $\sim 10^{6} \mathrm{~cm}^{-3}$ points to the existence of a hot, dense, compact core region at MM 1.

\subsubsection{Ortho- to Para- $\mathrm{H}_{2} \mathrm{CO}$ Ratio}

For the sources in which we have detected ortho- $\mathrm{H}_{2} \mathrm{CO}$ emission, we are able to estimate the $\mathrm{N}$ (ortho- $\left.\mathrm{H}_{2} \mathrm{CO}\right) /$ $N$ (para- $\left.\mathrm{H}_{2} \mathrm{CO}\right)$ ratio using our LVG model results. By applying our best-fit para- $\mathrm{H}_{2} \mathrm{CO}$-derived $n\left(\mathrm{H}_{2}\right)$ and $T_{K}$ (Table 5) with an ortho- $\mathrm{H}_{2} \mathrm{CO}$ LVG model, we can derive an LVG estimate of the ortho- $\mathrm{H}_{2} \mathrm{CO}$ transition intensities. The ortho$\mathrm{H}_{2} \mathrm{CO}$ models which reproduce our observed ortho- $\mathrm{H}_{2} \mathrm{CO}$ radiation temperatures imply that $N$ (ortho- $\left.\mathrm{H}_{2} \mathrm{CO}\right) / N$ (para$\left.\mathrm{H}_{2} \mathrm{CO}\right)=1.5,1.75$, and 3.0 in the Orion-KL hot core, ridge, and plateau sources, respectively; 3.0 in OMC-2 IRS 4 and NGC 2024; and 2.0 in NGC 2071 IRS 1, NGC 2264IR, IRAS 16293-2422, NGC 6334I, and G34.26+0.15. For DR $21(\mathrm{OH})$ we were able to constrain separate $\mathrm{LVG}$ models for ortho- and para- $\mathrm{H}_{2} \mathrm{CO}$ (see Table 5 ). From these two separate model solutions we derive $N\left(\right.$ ortho- $\left.\mathrm{H}_{2} \mathrm{CO}\right) / N\left(\right.$ para- $\left.\mathrm{H}_{2} \mathrm{CO}\right)=$ $1.78_{-0.28}^{+0.22}$ for DR $21(\mathrm{OH})$.

In the gas phase, the ortho and para forms of $\mathrm{H}_{2} \mathrm{CO}$ represent two distinct molecular species. This distinction comes about because rotational transitions between these two species require a highly improbable simultaneous transition of the nuclear spins. Interconversion between the two species can occur, however, if dust grains take an active role in $\mathrm{H}_{2} \mathrm{CO}$ chemistry. For $\mathrm{H}_{2} \mathrm{CO}$ molecules which either form or are adsorbed onto dust grains, hydrogen abstraction and addition reactions can alternately remove and replace hydrogen atoms by tunneling through modest potential barriers. This process allows the spins between the two species to be interconverted, which establishes ortho- and para- $\mathrm{H}_{2} \mathrm{CO}$ abundances at an equilibrium temperature equal to that of the grain surface (Kahane et al. 1984; Turner 1992). The relatively high kinetic temperature we derive $\left(T_{K} \gtrsim 50 \mathrm{~K}\right)$ and the typical ortho to para ratios of less than 3 indicate that $\mathrm{H}_{2} \mathrm{CO}$ gets adsorbed onto and desorbed from grain surfaces where interconversion takes place. Therefore, the measured ortho- to para- $\mathrm{H}_{2} \mathrm{CO}$ ratios in the molecular clouds in our sample indicate that dust grains play an important role in $\mathrm{H}_{2} \mathrm{CO}$ chemistry.

\section{DISCUSSION}

In the LVG analysis presented in $\S 4$, model solutions to the kinetic temperature, spatial density, and species- $\mathrm{H}_{2} \mathrm{CO}$ column density were derived for each object in our sample. Within the framework of the uniform, spherical LVG model we employ, there are two basic assumptions, equal source size for all transitions and the neglect of infrared excitation, which need to be addressed. In $\S \S 5.1$ and 5.2 we analyze the importance of these two assumptions in relation to our $\mathrm{LVG}$ model solutions. In $\S 5.3$ we present a synopsis of our $\mathrm{H}_{2} \mathrm{CO}$ LVG model results.

\subsection{Source Size}

Because we do not have information regarding the relative spatial distribution of the $\mathrm{H}_{2} \mathrm{CO}$ transitions for the sources in this analysis, we have assumed that the $\mathrm{H}_{2} \mathrm{CO}$ source size is the same for all transitions. Furthermore, in order to directly compare all of our $\mathrm{H}_{2} \mathrm{CO}$ measurements, we have converted all of our measured transition antenna temperatures to the telescope-independent $T_{R}$ scale ( see $\S 2$ ). Since we applied a mainbeam efficiency measured using Jupiter to convert $T_{A}^{*}$ and $T_{R}^{*}$ to $T_{R}$, our transition radiation temperatures are appropriate for a source size equal to that of Jupiter $\left(\simeq 40^{\prime \prime}\right)$. For many of our observed sources, measurements using other molecular tracers indicate that $\theta_{s}<40^{\prime \prime}$. To test the effect that the source size has on our model results, we have corrected our para$\mathrm{H}_{2} \mathrm{CO}$ measurements of DR $21(\mathrm{OH})$ for beam dilution assuming a source size equal to that derived from high-resolution $\mathrm{NH}_{3}$ measurements, 4" (Mangum et al. 1992). The derived $T_{K}$ from this test is the same as that found above, but the derived $n\left(\mathrm{H}_{2}\right)$ is smaller by approximately an order of magnitude. This decrease in $n\left(\mathrm{H}_{2}\right)$ is mainly due to the increased $\tau$ in the modeled transitions.

One can also envision a situation where the higher excitation transitions originate from a smaller volume within the molecular cloud. This would mean that the intensities of the higher excitation transitions are actually larger than we have assumed them to be. A radiation temperature ratio calculated under these circumstances would actually be an upper limit to the true value. If our assumption of equal source size in all $\mathrm{H}_{2} \mathrm{CO}$ transitions is not correct, the radiation temperature ratios shown in Figures 13, 14, and 15 indicate that our derived $T_{K}$ and $n\left(\mathrm{H}_{2}\right)$ are lower limits to their actual values.

Therefore, given a lack of knowledge regarding the relative spatial distribution of the $\mathrm{H}_{2} \mathrm{CO}$ transitions in this analysis, derived kinetic temperatures are at least lower limits to their true values, while the spatial densities derived are very sensitive to the assumed source size.

\subsection{Infrared Excitation}

\subsubsection{Near-Infrared Rovibrational Excitation}

In Appendix B we present a general method which one can use to calculate the spontaneous emission coefficient for a rovibrational transition of a symmetric or slightly asymmetric rotor molecule. This method is used to calculate the spontaneous emission coefficients for the rovibrational transitions of $\mathrm{H}_{2} \mathrm{CO}$, which we use to analyze the importance of near-infrared excitation to the analysis of $\mathrm{H}_{2} \mathrm{CO}$ rotational spectra 
(Appendix C). Using a model which is similar to that presented by Carroll \& Goldsmith (1981), in Appendix C we derive a general expression which gives the maximum size of a region in a molecular cloud which can produce near-infrared excitation of rovibrational transitions of any given molecule $\left(\theta_{1 \mathrm{R}}\right.$, eq. [C7]). In the presence of significant rovibrational excitation, spatial densities derived neglecting this effect are overestimated. Applying equation (C7) to the case of $\mathrm{H}_{2} \mathrm{CO}$, we find that for the sources in our sample with $L \simeq 10^{5} L_{\odot}$ and $D \gtrsim 1000$ pc [W3 IRS 4, W3(OH), NGC 6334I, G34.26+0.15, DR $21(\mathrm{OH})], \theta_{1 \mathrm{R}} \lesssim 10^{\prime \prime}$. For the sources with $L$ $\lesssim 5 \times 10^{3} L_{\odot}$ (OMC-2 IRS 4, NGC 2071 IRS 1, NGC 2264IR, IRAS 16293-2422), $\theta_{\mathrm{IR}} \lesssim 5^{\prime \prime}$. Since NGC 2024 and Orion-KL are nearby ( 415 and $480 \mathrm{pc}$, respectively) and luminous $\left(10^{4}\right.$ and $10^{5} L_{\odot}$, respectively), they possess relatively large regions over which near-infrared radiation can excite rovibrational transitions, $\theta_{\mathrm{IR}} \lesssim 15^{\prime \prime}$ and 40", respectively. In NGC 2024 and Orion-KL the region over which rovibrational excitation may be important approaches the spatial resolution of our measurements. Carroll \& Goldsmith (1981) concluded that, for the CS molecule, neglect of rovibrational transitions can result in overestimates of the spatial density of at most an order of magnitude. Similar overestimates may exist for spatial densities based on $\mathrm{H}_{2} \mathrm{CO}$ measurements of nearby, luminous sources which ignore rovibrational excitation. As higher resolution measurements of the dense molecular cores within active starforming regions become available, it will become necessary to include rovibrational excitation in the derivation of molecular cloud properties.

\subsubsection{Far-Infrared $\Delta K_{-1}=2$ Excitation}

Since the $\Delta K_{-1}=2$ transitions of $\mathrm{H}_{2} \mathrm{CO}$ lie at far-infrared wavelengths $\left[\nu(K=2 \rightarrow K=0) \simeq 32-60 \mathrm{~cm}^{-1}, \nu(K=3 \rightarrow K=\right.$ $\left.1) \simeq 61-90 \mathrm{~cm}^{-1}, \nu(K=4 \rightarrow K=2) \simeq 98-115 \mathrm{~cm}^{-1}\right)$, they can affect our analysis of the rotational energy levels of the ground vibrational state in the same manner as the rovibrational transitions. Therefore, we can apply equations (C4) and (C7) to the $\Delta K_{-1}=2$ transitions to derive the importance of far-infrared excitation to our analysis of the rotational excitation of $\mathrm{H}_{2} \mathrm{CO}$. For the $\Delta K_{-1}=2$ transitions, $A_{\mathrm{vib}}=A_{u l} \simeq 10^{-5} \mathrm{~s}^{-1}$ (for $K=3 \rightarrow 1$ and $K=4 \rightarrow 2$ ) to $10^{-6} \mathrm{~s}^{-1}$ (for $K=2 \rightarrow 0$ ). Note that collisional excitation is still negligible in this case, since the collisional rates for the $\Delta K_{-1}=2$ transitions are about an order of magnitude smaller than those for the $\Delta K_{-1}=1$ transitions. We find that the minimum infrared dust temperature necessary to excite the $\Delta K_{-1}=2$ transitions, $T_{\mathrm{IR}}(R)$, is $\sim 10^{3} \mathrm{~K}$ and $\theta_{\mathrm{IR}} \lesssim 15^{\prime \prime}$, indicating that far-infrared excitation of the $\Delta K_{-1}=$ 2 transitions of $\mathrm{H}_{2} \mathrm{CO}$ is negligible.

To check this simple analysis, we have made several LVG model calculations with an infrared radiation field added to the radiative transfer. In this analysis we assume a source filling factor of 1 , an infrared radiation field temperature of $100 \mathrm{~K}$, $n\left(\mathrm{H}_{2}\right)=10^{6} \mathrm{~cm}^{-3}, N\left(\right.$ para- $\left.\mathrm{H}_{2} \mathrm{CO}\right) / \Delta v=10^{14} \mathrm{~cm}^{-2}(\mathrm{~km}$ $\left.\mathrm{s}^{-1}\right)^{-1}$, and $T_{K}=100 \mathrm{~K}$. A comparison with models run under the same parameters and no infrared radiation field $(f=0)$ indicates that the addition of an infrared radiation field produces an overall shift of population from the lower $J$ to the upper $J$ states of a given $K_{-1}$ ladder, from the $K_{-1}=0$ to the $K_{-1}=2$, and from the $K_{-1}=2$ to the $K_{-1}=4$ ladders. This shift of population leads to an $\sim 10 \%-15 \%$ decrease in the radiation temperatures for transitions in the $K_{-1}=0$ and $K_{-1}=2$ ladders and a subsequent $\sim 10 \%$ increase for transitions in the $K_{-1}=4$ ladder. This implies that the introduction of an infrared radiation field to the rotational excitation produces an $\sim 5 \%$ increase in our derivation of $n\left(\mathrm{H}_{2}\right)$ and $T_{K}$ and an $\sim 10 \%$ increase in our derivation of $N\left(\right.$ para- $\left.\mathrm{H}_{2} \mathrm{CO}\right) / \Delta v$. Since the values for $T_{\mathrm{IR}}(R)$ and $f$ used in this analysis are rather extreme, the derived increases in $n\left(\mathrm{H}_{2}\right)$ and $T_{K}$ probably represent upper limits to the effects of far-infrared radiation on the rotational analysis of $\mathrm{H}_{2} \mathrm{CO}$ emission. Since the changes derived are comparable to or smaller than other uncertainties in our non-LTE analysis of $\mathrm{H}_{2} \mathrm{CO}$ excitation, infrared excitation of the $\Delta K_{-1}=2$ transitions is not significant.

\subsection{Synopsis of Model Results}

In $\S \S 4.5 .1 .1-4.5 .1 .11$ we compared our $\mathrm{H}_{2} \mathrm{CO}$ LVG model derivations of $T_{K}$ and $n\left(\mathrm{H}_{2}\right)$ with the known physical properties for each source in our sample. In all cases our derived $n\left(\mathrm{H}_{2}\right)$ is in good agreement with previous estimates. Note, however, that many of these previous estimates are based on simple critical density or size-column density calculations which yield only a very rough estimate for $n\left(\mathrm{H}_{2}\right)$. Coupled with our $T_{K}$ measurements, the spatial density we derive represents, for many of the sources in our sample, the most accurate $n\left(\mathrm{H}_{2}\right)$ measurement currently available.

The importance of measuring transitions whose ratios allow independent measurements of both $T_{K}$ and $n\left(\mathrm{H}_{2}\right)$ is apparent from our model analysis. Three of the poorest model fits in our analysis (IRAS 16293-2422, NGC 6334I, and G34.26+0.15) are constrained only by measurements from the $J=5 \rightarrow 4$ transitions. As a comparison of these measurements contains little information regarding the spatial density in these regions, this parameter is poorly constrained. The importance of obtaining measurements of the $J=3 \rightarrow 2$ transitions is apparent.

For many of the sources in our sample, we derive kinetic temperatures which are 2-6 times those estimated from previous measurements. In most of these sources previous $T_{K}$ estimates were based on measurements of low-excitation molecular transitions and/or cool dust continuum emission. For example, the $\mathrm{NH}_{3}(1,1)$ and $\mathrm{NH}_{3}(2,2)$ transitions, the ratio of which is one of the more traditional monitors of kinetic temperature in molecular clouds, lose sensitivity to kinetic temperature above $\sim 40 \mathrm{~K}$ (Walmsley \& Ungerechts 1983; Mangum et al. 1992). Therefore, these measurements suffered from a lack of information regarding the gas kinetic temperature at $T_{K} \gtrsim$ $50 \mathrm{~K}$. Since an accurate measurement of the kinetic temperature in a molecular cloud is fundamental to any measurement of the spatial density, our results point out the importance of using high-excitation molecular transitions to trace the physical conditions in warm star-forming regions.

In $\S \S 4$ and 5 we have analyzed many of the uncertainties inherent in our model analysis. In $\S 4.2$ we discussed the uncertainties in the collisional excitation rates used in our analysis of the excitation of $\mathrm{H}_{2} \mathrm{CO}$. Since the excitation rates are calculated for $\mathrm{H}_{2} \mathrm{CO}-\mathrm{He}$ collisions, as suggested by Green (1991) we have scaled all of our rates by a factor of 2.2 to account for the differences between $\mathrm{H}_{2}$ and $\mathrm{He}$ collisional excitation. Green points out that these $\mathrm{H}_{2} \mathrm{CO}$ collisional excitation rates are accu- 
rate to $\sim 20 \%$. We also analyzed the importance of the $\Delta K_{-1}=$ 2 transitions to our model analysis. Ignoring the effects of infrared excitation, exclusion of the $\Delta K_{-1}=2$ transitions results in an error of less than $20 \%$. In our $\mathrm{H}_{2} \mathrm{CO}$ model analysis we also ignored the effects due to infrared excitation of the rotational energy levels. Using a simple model to describe the infrared excitation in a dense molecular cloud, we found that far-infrared absorption through the $\Delta K_{-1}=2$ transitions is negligible. Near-infrared excitation of the rovibrational transitions of $\mathrm{H}_{2} \mathrm{CO}$, on the other hand, may be important in the most wellresolved and luminous star-forming regions.

The final uncertainty we addressed dealt with our assumption of an equal spatial distribution for all sources and $\mathrm{H}_{2} \mathrm{CO}$ transitions within these sources. We find that failure of this assumption would force our derived kinetic temperatures to become lower limits. Derived spatial densities are very sensitive to the assumed source size.

\section{CONCLUSIONS}

1. We have presented a detailed analysis of the kinetic temperature and spatial density probing properties of the rotational transitions of $\mathrm{H}_{2} \mathrm{CO}$. Using a spherical LVG model to describe the molecular excitation, we found that, over a range in physical conditions which encompasses that found in dense molecular clouds, the following are true:

a) Comparison of the intensities of $\mathrm{H}_{2} \mathrm{CO}$ transitions from different $K$-ladders within the same molecular species is an excellent monitor of kinetic temperature. We have analyzed the kinetic temperature sensitivity of several of these transition intensity ratios and derive rules of thumb for using them as probes of $T_{K}$ in molecular clouds. An LTE expression, which is applicable to any symmetric or slightly asymmetric rotor molecule (Appendix A), relating the kinetic temperature to the relative strengths of two such transitions is consistent with our LVG results over a limited range of physical conditions.

$b$ ) Following a specification of the kinetic temperature in a molecular cloud, comparison between the intensities of $\mathrm{H}_{2} \mathrm{CO}$ transitions from the same $K$-ladder is an excellent monitor of the spatial density in a molecular cloud. We derive several rules of thumb for using two of these ratios as spatial density probes.

We have applied this spherical LVG model to measurements of several $\mathrm{H}_{2} \mathrm{CO}$ transitions between 211 and $365 \mathrm{GHz}$ to derive the kinetic temperature and spatial density in a sample of active star-forming regions. In all of the regions in our sample we measure kinetic temperatures greater than $50 \mathrm{~K}$, significantly higher than previous estimates for many of these sources. Most measurements of the kinetic temperature in dense molecular clouds are based on measurements of low-excitation molecular transitions and/or cool dust continuum emission. The rotational transitions of $\mathrm{H}_{2} \mathrm{CO}$ are sensitive to a wide range of kinetic temperatures. Therefore, the use of $\mathrm{H}_{2} \mathrm{CO}$ as a kinetic temperature probe represents a significant advancement in our ability to measure gas kinetic temperatures in dense molecular clouds.

2. We have conducted a detailed analysis of the uncertainties due to the accuracy of the collisional excitation rates, the neglect of infrared excitation, and our assumptions regarding source angular size. The first two of these factors produce errors smaller than $20 \%$ in our derived physical parameters. Failure of our assumptions regarding source angular size implies that our derived kinetic temperatures are at least lower limits to their actual values. The derived spatial density is very sensitive to assumptions regarding source size.

3. Using our ortho- and para- $\mathrm{H}_{2} \mathrm{CO}$ measurements, we have calculated the $N$ (ortho- $\left.\mathrm{H}_{2} \mathrm{CO}\right) / N\left(\right.$ para- $\left.\mathrm{H}_{2} \mathrm{CO}\right)$ ratio in several of the sources in our sample. Our measurements indicate that $N\left(\right.$ ortho- $\left.\mathrm{H}_{2} \mathrm{CO}\right) / N\left(\right.$ para- $\left.\mathrm{H}_{2} \mathrm{CO}\right)<3$ for most of our sources. When combined with the relatively high kinetic temperatures in these objects, this $N\left(\right.$ ortho- $\left.\mathrm{H}_{2} \mathrm{CO}\right) / N($ para$\mathrm{H}_{2} \mathrm{CO}$ ) ratio indicates that dust grains play an active role in $\mathrm{H}_{2} \mathrm{CO}$ chemistry.

4. A general method is described whereby one can calculate the spontaneous emission coefficient for a rovibrational transition of any symmetric or slightly asymmetric rotor molecule. We have applied this method to the rovibrational transitions of $\mathrm{H}_{2} \mathrm{CO}$.

5. Following the formalism set forth by Carroll \& Goldsmith (1981), we derive a general expression which estimates the maximum angular size of an infrared stimulated region in a molecular cloud. We find that near-infrared excitation of the rovibrational transitions of $\mathrm{H}_{2} \mathrm{CO}$ could significantly alter the level populations for the most luminous and nearby star-forming regions.

6. Since $\mathrm{H}_{2} \mathrm{CO}$ is free of large-scale chemical inhomogeneities in the interstellar medium, through the analysis in this paper we have shown that it is the most unbiased and potentially accurate probe of kinetic temperature and spatial density in the interstellar medium.

J.G.M. would like to thank the National Radio Astronomy Observatory for support through a Junior Research Associateship. We are grateful for Todd Groesbeck's assistance with the observations. We would also like to thank Barry Turner, Lee Mundy, and Neal Evans for providing useful insight into several aspects of this work. We also thank Neal Evans for carefully reading and commenting on the manuscript. This work was supported in part by Texas Advanced Research Program grant 003658-285 and National Science Foundation grant AST-9017710 to the University of Texas. The CSO is supported by the National Science Foundation under contract AST90-15755.

\section{APPENDIX A}

Since $\mathrm{H}_{2} \mathrm{CO}$ is nearly a symmetric rotor molecule, different $K$-ladders within the same $\mathrm{H}_{2} \mathrm{CO}$ species are connected primarily by collisions (there are weak radiative transitions which are relatively unimportant; see $\S 4.2$ ). Therefore, the relative population occupying the ground states from these two $K$-ladders are related through the Boltzmann equation at kinetic temperature $T_{K}$. Since one generally does not measure the ground-state transition, it becomes necessary to see whether other transitions within the $K$-ladders are related by the Boltzmann equation. In the following, we will derive such a relation and analyze the uncertainties 
encountered when using it to derive the kinetic temperature in a molecular cloud. The derivation we present should also be applicable to any slightly asymmetric or symmetric rotor molecule.

To begin, we make the following definitions:

$(J, K) \equiv$ Upper energy state of first transition in comparison.

$\left(J, K^{\prime}\right) \equiv$ Upper energy state of second transition in comparison $\left(K^{\prime}>K\right)$.

$(K, K) \equiv$ Ground energy state of the first transition in comparison.

$\left(K^{\prime}, K^{\prime}\right) \equiv$ Ground energy state of the second transition in comparison.

$T_{J J} \equiv$ Excitation temperature between levels $\left(J, K^{\prime}\right)$ and $(J, K)$.

$T_{J K^{\prime}} \equiv$ Excitation temperature between levels $\left(J, K^{\prime}\right)$ and $\left(K^{\prime}, K^{\prime}\right)$.

$T_{J K} \equiv$ Excitation temperature between levels $(J, K)$ and $(K, K)$.

$T_{K K^{\prime}} \equiv$ Excitation temperature between levels $(K, K)$ and $\left(K^{\prime}, K^{\prime}\right)$.

The populations of the four energy levels in this comparison are related by the following:

$$
\frac{n\left(J, K^{\prime}\right)}{n(J, K)}=\left[\frac{n\left(J, K^{\prime}\right)}{n\left(K^{\prime}, K^{\prime}\right)}\right]\left[\frac{n\left(K^{\prime}, K^{\prime}\right)}{n(K, K)}\right]\left[\frac{n(K, K)}{n(J, K)}\right] .
$$

Using the Boltzmann equation,

$$
\frac{n\left(J, K^{\prime}\right)}{n(J, K)}=\frac{g\left(J, K^{\prime}\right)}{g(J, K)} \exp \left[-\frac{E\left(J, K^{\prime}\right)-E(J, K)}{T_{J J}}\right],
$$

assuming that the excitation temperature of the $\Delta J=1$ transitions in both $K$-ladders is the same $\left(T_{J K}=T_{J K^{\prime}}\right)$, and noting that $T_{K K^{\prime}}$ is also the kinetic temperature in the gas $\left(T_{K}\right)$, we find that

$$
\frac{T_{K}}{T_{J J}}=\frac{E\left(K^{\prime}, K^{\prime}\right)-E\left(K^{\prime}, K\right)}{E\left(J, K^{\prime}\right)-E(J, K)}-\frac{E\left(K^{\prime}, K\right)-E(K, K)}{E\left(J, K^{\prime}\right)-E(J, K)}\left(\frac{T_{K}}{T_{J K}}-1\right) .
$$

To very good approximation,

$$
E\left(J, K^{\prime}\right)-E(J, K) \simeq E\left(K^{\prime}, K^{\prime}\right)-E\left(K^{\prime}, K\right)
$$

For most heavy symmetric tops (e.g., $\mathrm{CH}_{3} \mathrm{CN}$ ), it is also true that

$$
E\left(K^{\prime}, K\right)-E(K, K) \ll E\left(K^{\prime}, K^{\prime}\right)-E\left(K^{\prime}, K\right)
$$

But, because the energy levels in $\mathrm{H}_{2} \mathrm{CO}$ are more widely spaced than they are for many heavy symmetric tops, this approximation is not quite valid. Thus, for $\mathrm{H}_{2} \mathrm{CO}$ the equation above reduces to

$$
\frac{T_{K}}{T_{J J}}=1-\frac{E\left(K^{\prime}, K\right)-E(K, K)}{E\left(K^{\prime}, K^{\prime}\right)-E\left(K^{\prime}, K\right)}\left(\frac{T_{K}}{T_{J K}}-1\right) .
$$

Comparison of the $K=0$ and $2, K=1$ and 3, and $K=2$ and 4 relative energy-level separations indicates that for $T_{K} / T_{J K} \lesssim 2.0, T_{J J}$ overpredicts $T_{K}$ by $\lesssim 30 \%$.

Now that we have found the conditions under which the kinetic temperature is approximately equal to the excitation temperature between levels $(J, K)$ and $\left(J, K^{\prime}\right)$, we can relate $T_{K}$ to the measured radiation temperatures $T_{R}(J, K)$ and $T_{R}\left(J, K^{\prime}\right)$. The LTE column density in level $(J, K)$ is given by

$$
N(J, K)=\frac{3 h g_{J}}{8 \pi^{3}\left|\mu_{J K}\right|^{2}}\left[\exp \left(\frac{h \nu}{k T_{J K}}\right)-1\right]^{-1} \int \tau(J, K) d v
$$

and the radiation temperature $T_{R}$ is related to $T_{\mathrm{ex}}$ by

$$
J\left(T_{R}\right)=\left[J\left(T_{\mathrm{ex}}\right)-J\left(T_{\mathrm{cmb}}\right)\right][1-\exp (-\tau)],
$$

where

$$
J(T)=\frac{h \nu}{k}\left[\exp \left(\frac{h \nu}{k T}\right)-1\right]^{-1}
$$


Assuming that both transitions are optically thin and that $T_{\mathrm{cmb}}$ is negligible, $N(J, K)$ can be written

$$
N(J, K)=\frac{3 k g_{J}}{8 \pi^{3} \nu(J, K)\left|\mu_{J K}\right|^{2}} \int J\left(T_{R}\right) d v .
$$

Then since $\nu(J, K) \simeq \nu\left(J, K^{\prime}\right)$ and

$$
\left|\mu_{J K}\right|^{2}=S_{J K} \mu^{2},
$$

where $S_{J K}$ is the transition line strength, we can use the Boltzmann equation to relate the column density ratio $N\left(J, K^{\prime}\right) / N(J, K)$ to the kinetic temperature $T_{K}$ :

$$
\frac{S_{J K} \int J\left(T_{R}\left(J, K^{\prime}\right)\right) d v}{S_{J K^{\prime}} \int J\left(T_{R}(J, K)\right) d v}=\frac{g\left(J, K^{\prime}\right)}{g(J, K)} \exp \left[-\frac{E\left(J, K^{\prime}\right)-E(J, K)}{T_{K}}\right] .
$$

Then, since the level degeneracies $g_{J K}$ and $g_{J K^{\prime}}$ are given by $g_{J} g_{K}$ and $g_{J} g_{K^{\prime}}$, respectively, and $g_{K}=g_{K^{\prime}}=1$ (no $K$-degeneracy in asymmetric rotor molecules), we find that

$$
T_{K}=\left[E\left(J, K^{\prime}\right)-E(J, K)\right]\left\{\ln \left[\frac{S_{J K^{\prime}} \int J\left(T_{R}(J, K)\right) d v}{S_{J K} \int J\left(T_{R}\left(J, K^{\prime}\right)\right) d v}\right]\right\}^{-1} .
$$

The line strengths and energies for the $\mathrm{H}_{2} \mathrm{CO}$ transitions discussed in this paper are given in Table 2 .

The critical assumptions used to derive this expression are the following:

1. $\tau \ll 1$ : For the observed para- $\mathrm{H}_{2} \mathrm{CO}$ transitions measured in the current study, $\tau \lesssim 1$.

2. $T_{J K} \simeq T_{J K^{\prime}}$ : These excitation temperatures differ by less than a factor of 2 over the range of physical conditions that we have studied.

3. $T_{K} / T_{J K} \leqslant 2$ : Good for $n\left(\mathrm{H}_{2}\right) \gtrsim 10^{6.5} \mathrm{~cm}^{-3}$.

\section{APPENDIX B}

In the following we describe a general method with which one can calculate the spontaneous emission coefficient for a rovibrational transition of a symmetric or slightly asymmetric rotor molecule. Detailed information regarding the calculations presented in this appendix can be found in Hönl \& London (1925, 1926), Herzberg (1945), Townes \& Schawlow (1975), Demtroder (1981), Person \& Zerbi (1982), and Gordy \& Cook (1984). For a rovibrational transition, within mode $i$, between upper state $u$ and lower state $l$ with frequency $\nu\left(\mathrm{cm}^{-1}\right)$, the spontaneous emission coefficient is given by

$$
A_{u l}=\frac{64 \pi^{4} \nu^{3}}{3 h g_{u} c^{3}} L\left(J_{l}, K_{l}\right)\left|\mu_{v_{l}}\right|^{2},
$$

where $g_{u}=2 J_{u}+1$ is the upper-state rotational degeneracy, $L\left(J_{l}, K_{l}\right)$ is the Hönl-London factor for the lower state $\left(J_{l}, K_{l}\right)$, and $\left|\mu_{v_{l}}\right|$ is the vibrational matrix element for band $v_{i}$. In this expression we assume that corrections due to rotation-vibration interactions are negligible.

To calculate $L\left(J_{l}, K_{l}\right)$ for the general asymmetric rotor molecule, one must sum the squares of the dipole moment matrix elements over the three space-fixed axes of the molecule. In general, it is not possible to obtain a closed expression for $L\left(J_{l}, K_{l}\right)$ as a function of $J_{l}$ and $K_{l}$. Therefore, the usual way for deriving $L\left(J_{l}, K_{l}\right)$ for asymmetric rotors is to use the limiting symmetric rotor function and apply a correction factor based on the degree of asymmetry for the molecule. Since formaldehyde is a slightly asymmetric rotor molecule $(\kappa=-0.96)$, these corrections to the symmetric rotor Hönl-London factors are small, so we have chosen to neglect them in this analysis.

In the following calculations we assume a rovibrational transition from the $v=0$ to the $v=1$ state. For a symmetric rotor molecule transition where $\Delta K=0$,

$$
L\left(J_{l}, K_{l}\right)= \begin{cases}\frac{\left(J_{l}+1\right)^{2}-K_{l}^{2}}{J_{l}+1}, & b=R(\Delta J=+1), \\ \frac{\left(2 J_{l}+1\right) K_{l}^{2}}{J_{l}\left(J_{l}+1\right)}, & b=Q(\Delta J=0), \\ \frac{J_{l}^{2}-K_{l}^{2}}{J_{l}}, & b=P(\Delta J=-1),\end{cases}
$$


while for $\Delta K= \pm 1$,

$$
L\left(J_{l}, K_{l}\right)= \begin{cases}\frac{\left(J_{l}+2 \pm K_{l}\right)\left(J_{l}+1 \pm K_{l}\right)}{4\left(J_{l}+1\right)}, & b=R(\Delta J=+1), \\ \frac{\left(2 J_{l}+1\right)\left(J_{l}+1 \pm K_{l}\right)\left(J_{l} \mp K_{l}\right)}{4 J_{l}\left(J_{l}+1\right)}, & b=Q(\Delta J=0), \\ \frac{\left(J_{l}-1 \mp K_{l}\right)\left(J_{l} \mp K_{l}\right)}{4 J_{l}}, & b=P(\Delta J=-1),\end{cases}
$$

where the upper sign refers to $\Delta K=+1$, the lower to $\Delta K=-1$, and $b$ indicates the transition branch (Lide 1952). For $\Delta K= \pm 1$ the Hönl-London factors given above are $\frac{1}{4}$ of those given by Herzberg (1945), but this difference affects only the vibrational dipole moment and has no affect on the calculation of the Einstein $A$-value. Note that for $\Delta K= \pm 1$ and $K_{l}=0, L\left(J_{l}, K_{l}\right)$ must be multiplied by 2 to account for the fact that the statistical weight for $K_{l}=0$ is only half that for $K_{l} \neq 0$.

Evans, Lacy, \& Carr (1991) describe how to calculate $\left|\mu_{v_{l}}\right|$ from laboratory measurements of the band strength $S_{v}^{i}$ :

$$
\left|\mu_{v_{l}}\right|^{2}=\frac{3 h c}{8 \pi^{3}} \frac{Q_{v}\left(T_{i}\right)}{\nu_{i}\left[1-\exp \left(-h c v_{i} / k T_{i}\right)\right]} S_{v}^{i}\left[\sum_{b} \frac{L\left(J_{l}, K_{l}\right)}{g_{J_{l}} g_{K_{l}}}\right]^{-1}
$$

where

$$
\begin{aligned}
g_{J_{l}} & =2 J_{l}+1, \\
g_{K_{l}} & = \begin{cases}1 & \text { for } K_{l}=0, \\
2 & \text { for } K_{l} \neq 0,\end{cases} \\
\nu_{i} & =\text { band center frequency in } \mathrm{cm}^{-1}, \\
T_{i} & =\text { band vibrational temperature in } \mathrm{K}, \\
Q_{v}\left(T_{i}\right) & =\text { vibrational partition function, }
\end{aligned}
$$

and the sum is over all branches $b$. Note that the frequency in this calculation is the band-center frequency because we wish to calculate an average over the entire band. The vibrational partition function $Q_{v}\left(T_{i}\right)$ is given by

$$
Q_{v}\left(T_{t}\right)=\prod_{i}\left[1-\exp \left(\frac{-h c \nu_{i}}{k T_{i}}\right)\right]^{-d_{i}},
$$

where $d_{i}$ is the degeneracy of band $i$. For the fundamental vibration bands in $\mathrm{H}_{2} \mathrm{CO}, d_{i}=1$. As Evans et al. (1991) point out, this expression is valid only if we neglect anharmonicity, which is justified only for the lower vibrational states. For laboratory measurements, $T_{i}$ is equal to the ambient temperature in the laboratory (commonly assumed to be $300 \mathrm{~K}$ ).

TABLE 6

ROVIBRATIONAL TRANSITIONS $\mathrm{OF}_{2} \mathrm{CO}$

\begin{tabular}{lccccr}
\hline \hline \multicolumn{1}{c}{ Band $^{\mathrm{a}}$} & $\begin{array}{c}\text { Band Type } \\
(\Delta K)\end{array}$ & $\begin{array}{c}\nu_{i}^{\mathrm{c}} \\
\left(\mathrm{cm}^{-1}\right)\end{array}$ & $\begin{array}{c}S_{-v}^{i \mathbf{d}} \\
\left.\left[10^{-17} \mathrm{~cm}^{-1}\left(\mathrm{~cm}^{-2}\right)^{-1}\right)\right]\end{array}$ & $\begin{array}{c}\left|\mu_{v_{t}}\right| \\
(\text { debye })\end{array}$ & $\begin{array}{r}A_{\mu_{l}}^{\mathrm{e}} \\
\left(\mathrm{s}^{-1}\right)\end{array}$ \\
\hline$v_{6}\left(b_{2}\right) \ldots \ldots$ & \pm 1 & 1167.258 & $0.080 \pm 0.002$ & $0.057 \pm 0.009$ & 1.64 \\
$v_{5}\left(b_{1}\right) \ldots \ldots$ & \pm 1 & 1249.091 & $0.136 \pm 0.004$ & $0.072 \pm 0.012$ & 3.19 \\
$v_{3}\left(a_{1}\right) \ldots \ldots$ & 0 & 1500.176 & $0.192 \pm 0.005$ & $0.055 \pm 0.009$ & 3.25 \\
$v_{2}\left(a_{1}\right) \ldots \ldots$ & 0 & 1746.0089 & $1.229 \pm 0.088$ & $0.130 \pm 0.035$ & 28.21 \\
$v_{1}\left(a_{1}\right) \ldots \ldots$ & 0 & 2782.4569 & $1.254 \pm 0.117$ & $0.104 \pm 0.032$ & 73.07 \\
$v_{4}\left(b_{1}\right) \ldots \ldots$ & \pm 1 & 2843.3256 & $1.454 \pm 0.133$ & $0.157 \pm 0.047$ & 177.02 \\
\hline
\end{tabular}

${ }^{a}$ Band designation according to the notation convention of Herzberg 1945.

${ }^{\mathrm{b}}$ Band type is also referred to as parallel $(\Delta K=0)$ and perpendicular $(\Delta K= \pm 1)$.

${ }^{\mathrm{c}}$ Band-center frequencies $\nu_{i}$ are from Clouthier \& Ramsay 1983.

${ }^{\mathrm{d}}$ Band strengths $S_{v}^{i}$ for $v_{2}, v_{1}$, and $v_{4}$ from Nakanaga, Kondo, \& Saëki 1982; for $v_{6}, v_{5}$, and $v_{3}$, from Reuter et al. 1989.

${ }^{\mathrm{e}}$ Einstein $A$ calculated for the $R(0,0)$ transition. 
Table 6 lists our calculations of $\left|\mu_{v_{i}}\right|$ and $A_{u l}$ from measured values for $S_{v}^{i}$ for all six fundamental vibration modes in $\mathrm{H}_{2} \mathrm{CO}$. Note that the quantum mechanical sum rules require that the sum over the $L\left(J_{l}, K_{l}\right)$ in the asymmetric rotor case for a given dipole transition is the same as that for the equivalent symmetric rotor. Therefore,

$$
\sum_{b} \frac{L\left(J_{l}, K_{l}\right)}{g_{J_{l}} g_{K_{l}}}= \begin{cases}\frac{1}{g_{K_{l}}}, & \Delta K=0, \\ \frac{1}{2 g_{K_{l}}}, & \Delta K= \pm 1 .\end{cases}
$$

APPENDIX C

In our analysis of the excitation of formaldehyde in molecular clouds, we solved the coupled equations of statistical equilibrium and radiative transfer for the rotational levels of the ground vibrational state $(v=0)$. This analysis neglected the influences of near-infrared absorption by vibrational transitions and far-infrared absorption by $\Delta K_{-1}=2$ rotational transitions. Since both of these processes can change the distribution of population in the rotational energy levels of the ground vibrational state, it is useful to analyze how infrared absorption can change our solutions for the physical properties of molecular cloud cores. In the following we will derive an expression which quantifies the importance of near-infrared absorption through rovibrational states. Note, however, that this expression will be quite general and will be applicable to any infrared excitation process.

Morris (1975) and Carroll \& Goldsmith (1981) found that transitions between the vibrational levels of several linear molecules can significantly change the populations of the upper rotational levels of the ground vibrational state in these molecules. Following the analysis of Carroll \& Goldsmith, the condition under which rovibrational excitation can significantly alter the populations of the ground vibrational state is given by

$$
B_{\mathrm{vib}} U+C_{\mathrm{vib}} n\left(\mathrm{H}_{2}\right) \geq A_{\text {rot }}+C_{\mathrm{rot}} n\left(\mathrm{H}_{2}\right),
$$

where $B_{\text {vib }}$ is the Einstein induced absorption coefficient due to absorption of infrared radiation through rovibrational excitation, $U$ is the radiation energy density at the rovibrational frequency $\nu_{\mathrm{vib}}, C_{\mathrm{vib}}$ is the collisional excitation rate for rovibrational transitions, $n\left(\mathrm{H}_{2}\right)$ is the molecular hydrogen spatial density, $A_{\text {rot }}$ is the Einstein spontaneous rotational de-excitation rate, and $C_{\text {rot }}$ is the collisional de-excitation rate for rotational transitions. We assume that induced emission is negligible. In general, collisional de-excitation between vibrational states is very slow $\left(C_{\mathrm{vib}} \leq 10^{-15} \mathrm{~cm}^{3} \mathrm{~s}^{-1}\right.$; Flower 1990), making the rovibrational collision term negligible. Assuming that the molecules of interest are influenced by an infrared radiation field from an embedded source of radius $R$ which can be characterized by a temperature $T_{\mathrm{IR}}(R)$ and emissivity $\epsilon_{\nu}$ diluted by a geometrical filling factor $f$, we can write the left-hand side of equation $(\mathrm{Cl})$ as

$$
B_{\mathrm{vib}} U \simeq \epsilon_{\nu} f A_{\mathrm{vib}}\left\{\exp \left[\frac{h c \nu_{\mathrm{vib}}}{k T_{\mathrm{IR}}(R)}\right]-1\right\}^{-1}
$$

which means that equation $(\mathrm{C} 1)$ becomes

$$
\epsilon_{\nu} f A_{\mathrm{vib}}\left\{\exp \left[\frac{h c \nu_{\mathrm{vib}}}{k T_{\mathrm{IR}}(R)}\right]-1\right\}^{-1} \geq A_{\mathrm{rot}}+C_{\mathrm{rot}} n\left(\mathrm{H}_{2}\right) .
$$

At this point we can obtain a zeroth-order estimate of the importance of rovibrational excitation. By setting $\epsilon_{\nu}$ and $f$ to their maximum value of 1 , we can solve equation $(\mathrm{C} 3)$ for $T_{\mathrm{IR}}(R)$ to obtain the minimum infrared source temperature necessary to excite the rovibrational transitions:

$$
T_{\mathrm{IR}}^{\min }(R) \geq \frac{h c \nu_{\mathrm{vib}}}{k}\left\{\ln \left[\frac{A_{\mathrm{vib}}}{A_{\mathrm{rot}}+C_{\mathrm{rot}} n\left(\mathrm{H}_{2}\right)}+1\right]\right\}^{-1} .
$$

For the $P$-branch rotational transitions of $\mathrm{H}_{2} \mathrm{CO}, A_{\text {rot }}+C_{\text {rot }} n\left(\mathrm{H}_{2}\right) \gtrsim 1.5 \times 10^{-5} \mathrm{~s}^{-1}$ assuming $n\left(\mathrm{H}_{2}\right)=10^{5} \mathrm{~cm}^{-3}$ and $T_{K}=100 \mathrm{~K}$. Using the data in Table 6, we find that $T_{\mathrm{IR}}^{\mathrm{min}}(R)=144-250 \mathrm{~K}$ for the rovibrational transitions of $\mathrm{H}_{2} \mathrm{CO}$. Since these temperatures are typical of many active star-forming regions, excitation of the rovibrational transitions of $\mathrm{H}_{2} \mathrm{CO}$ can occur.

Now that we have determined that the rovibrational transitions can be excited in molecular cloud cores, we need to derive an expression which will tell us the size of the region affected by infrared excitation of these transitions. For an embedded infrared source of radius $R$ located a distance $r$ from a molecular cloud, the source filling factor $f$ is given by

$$
f= \begin{cases}R^{2} / 2 r^{2} & r \geq R \\ 1 & \text { for } r<R .\end{cases}
$$


If we also assume that the dust emissivity behaves as a power law in frequency,

$$
\epsilon_{\nu}=\epsilon_{200}\left(\frac{\nu_{\mathrm{vib}}}{200}\right)^{\beta}, \quad \beta=1-2,
$$

we only need to specify the functional dependence of the infrared source temperature $T_{\mathrm{IR}}(R)$. In the infrared source model of Scoville \& Kwan (1976), an upper limit to $T_{\mathrm{IR}}(R)$ is obtained if one assumes that the dust emission is optically thin. Assuming $\beta=2$ (using $\beta=1$ results in a less than $10 \%$ change in our final result), Scoville $\&$ Kwan find that the maximum infrared dust temperature as a function of $R$ is given by

$$
T_{\mathrm{IR}}(R)=\frac{51}{\epsilon_{200}^{1 / 6}}\left(\frac{2 \times 10^{17} \mathrm{~cm}}{R}\right)^{1 / 3}\left(\frac{L_{*}}{10^{5} L_{\odot}}\right)^{1 / 6} .
$$

Substituting and solving equation (C3) for $r$ yields

$$
r \leq\left[\frac{\delta R^{2}}{\exp \left(\gamma R^{1 / 3}\right)-1}\right]^{1 / 2}
$$

where we have defined

$$
\begin{aligned}
& \gamma \equiv \frac{\epsilon_{200}^{1 / 6} h \nu_{\mathrm{vib}} c}{51 k}\left(\frac{1}{2 \times 10^{17} \mathrm{~cm}}\right)^{1 / 3}\left(\frac{10^{5} L_{\odot}}{L_{*}}\right)^{1 / 6}, \\
& \delta \equiv \frac{\epsilon_{200}}{2}\left[\frac{A_{\mathrm{vib}}}{A_{\mathrm{rot}}+C_{\mathrm{rot}} n\left(\mathrm{H}_{2}\right)}\right]\left(\frac{\nu_{\mathrm{vib}}}{200}\right)^{2} .
\end{aligned}
$$

The right-hand side of equation (C5) has a maximum at $\gamma R^{1 / 3}=6$. Plugging this maximum value back into equation (C5) yields

$$
r \leq\left[\left(\frac{6}{\gamma}\right)^{6}\left(\frac{\delta}{e^{6}-1}\right)\right]^{1 / 2}
$$

A molecule must be at least this close to an embedded infrared source for the infrared radiation field to significantly alter the level populations through infrared excitation. Putting equation (C6) into more useful units,

$$
\theta_{\mathrm{IR}}=2 r \lesssim \frac{4.527 \times 10^{7}}{D_{c}(\mathrm{pc}) \nu_{\mathrm{vib}}^{2}}\left\{\left(\frac{L_{*}}{10^{5} L_{\odot}}\right)\left[\frac{A_{\mathrm{vib}}}{A_{\mathrm{rot}}+C_{\mathrm{rot}} n\left(\mathrm{H}_{2}\right)}\right]\right\}^{1 / 2} \operatorname{arcsec}
$$

where $D_{c}$ is the distance to the molecular cloud in parsecs and $\nu_{\text {vib }}$ is the rovibrational frequency in inverse centimeters. Therefore, $\theta_{\mathrm{IR}}$ represents the angular size of the infrared stimulated region.

Allen, D. A. 1972, ApJ, 172, L55

Anthony-Twarog, B. J. 1982, AJ, 87, 1213

Bachiller, R., \& Cernicharo, J. 1990, A\&A, 239, 276

Bally, J. 1982, ApJ, 261, 558

Batrla, W., Wilson, T. L., Bastien, P., \& Ruf, K. 1983, A\&A, 128, 279

Blake, G. A., Sutton, E. C., Masson, C. R., \& Phillips, T. G. 1987, ApJ, 315,621

Butner, H. M., Evans, N. J., II, Harvey, P. M., Mundy, L. G., Natta, A., \& Randich, M. S. 1990, ApJ, 364, 164

Campbell, M. F., Hoffmann, W. F., Thronson, H. A., Jr., Niles, D., Nawfel, R., \& Hawrylycz, M. 1982, ApJ, 261, 550

Campbell, M. F., Lester, D. F., Harvey, P. M., \& Joy, M. 1989, ApJ, 345, 298

Carroll, T. J., \& Goldsmith, P. F. 1981, ApJ, 245, 891

Cesaroni, R., Walmsley, C. M., Kömpe, C., \& Churchwell, E. 1991, A\&A, 252,278

Churchwell, E., Walmsley, C. M., \& Wood, D. O. S. 1992, A\&A, 253, 541

Clouthier, D. J., \& Ramsay, D. A. 1983, Ann. Rev. Phys. Chem., 34, 31

Cornet, R., \& Winnewisser, G. 1980, J. Molec. Spectrosc., 80, 438

Demtroder, W. 1981, Laser Spectroscopy: Basic Concepts and Instrumentation (Berlin: Springer-Verlag), chaps. 1 and 2

\section{REFERENCES}

Downes, D., Wilson, T. L., Bieging, J., \& Wink, J. 1980, A\&AS, 40, 379

Dreher, J. W., \& Welch, W. J. 1981, ApJ, 245, 857

Ellison, B. N., Schaffer, P. L., Schaal, W., Vail, D., \& Miller, R. E. 1989, Int. J. Infrared Millimeter Waves, 10, 937

Evans, N. J., II, Beckwith, S., Brown, R. L., \& Gilmore, W. 1979, ApJ, 227, 450

Evans, N. J., II, Lacy, J. H., \& Carr, J. S. 1991, ApJ, 383, 674

Fischer, J., Righini-Cohen, G., \& Simon, M. 1980, ApJ, 238, L155

Fischer, J., Sanders, D. B., Simon, M., \& Solomon, P. M. 1985, ApJ, 293, 508

Flower, D. 1990, Molecular Collisions in the Interstellar Medium (Cambridge: Cambridge Univ. Press)

Garay, G., Reid, M. J., \& Moran, J. M. 1985, ApJ, 289, 681

Gaume, R. A., Johnston, K. J., \& Wilson, T. L. 1992, ApJ, 388, 489

Gaume, R. A., \& Mutel, R. L. 1987, ApJS, 65, 193

Gear, W. K., Chandler, C. J., Moore, T. J. T., Cunningham, C. T., \& Duncan, W. D. 1988, MNRAS, 231, 47P

Genzel, R. 1991, in The Physics of Star Formation and Early Stellar Evolution, ed. C. J. Lada \& N. D. Kylafis (Dordrecht: Kluwer), 155

Genzel, R., \& Downes, D. 1977, A\&A, 61, 117

- 1979, A\&A, 72, 234 
Genzel, R., Reid, M. J., Moran, J. M., \& Downes, D. 1981, ApJ, 244, 884

$\because$ Genzel, R., \& Stutzki, J. 1989, ARA\&A, 27, 41

Gordon, M. A. 1987, ApJ, 316, 258

Gordy, W., \& Cook, R. L. 1984, Microwave Molecular Spectra (New York: Wiley)

Green, S. 1991, ApJS, 76, 979

Griffin, M. J., Ade, P. A. R., Orton, G. S., Robson, E. I., Gear, W. K., Nolt, I. G., \& Radostitz, J. V. 1986, Icarus, 65, 244

Grossman, E. 1989, Atmospheric Transmission Software, Version 1.5

Harris, S., \& Wynn-Williams, C. G. 1976, MNRAS, 174, 649

Harvey, P. M., Campbell, M. F., \& Hoffman, W. F. 1977, ApJ, 215, 151

Harvey, P. M., Campbell, M. F., Hoffman, W. F., \& Thronson, H. A., Jr. 1979, ApJ, 229, 990

Harvey, P. M., \& Gatley, I. 1983, ApJ, 269, 613

Harvey, P. M., Joy, M., Lester, D. F., \& Wilking, B. A. 1986, ApJ, 300, 737

Hayashi, M., Kobayashi, H., \& Hasegawa, T. 1989, ApJ, 340, 298

Herzberg, G. 1945, Infrared and Raman Spectra (New York: Van Nostrand Reinhold)

Hönl, H., \& London, F. 1925, Z. Phys., 33, 803

1926, Ann. d. Phys., 79, 273

Jackson, J. M., Ho, P. T. P., \& Haschick, A. D. 1988, ApJ, 333, L73

Johansson, L. E. B., Hoglund, B., Winnberg, A., Rieu, N. Q., \& Goss, W. M. 1974, ApJ, 189, 455

Johnson, J. J., Gehrz, R. D., Jones, T. J., Hackwell, J. A., \& Grasdalen, G. L. 1990, AJ, 100, 518

Kahane, C., Frerking, M. A., Langer, W. D., Encrenaz, P., \& Lucas, R. 1984, A\&A, 137, 211

Kutner, M. L., \& Ulich, B. L. 1981, ApJ, 250, 341

Lane, A. P., \& Bally, J. 1986, ApJ, 310, 820

Lichten, S. M. 1982, ApJ, 253, 593

Lide, D. R. 1952, J. Chem. Phys., 20, 1761

Loughran, L., McBreen, B., Fazio, G. G., Rengarajan, T. N., Maxson, C. W., Serio, S., Sciortino, S., \& Ray, T. P. 1986, ApJ, 303, 629

Mangum, J. G., Wootten, A., Loren, R. B., \& Wadiak, E. J. 1990, ApJ, 348,542

Mangum, J. G., Wootten, A., \& Mundy, L. G. 1991, ApJ, 378, 576

1992, ApJ, 388, 467

Mangum, J. G., Wootten, A., \& Plambeck, R. L. 1993, ApJ, 409, 282

Margulis, M., Lada, C. J., \& Snell, R. L. 1988, ApJ, 333, 316

Mauersberger, R., Wilson, T. L., \& Henkel, C. 1988, A\&A, 201, 123

Mauersberger, R., Wilson, T. L., Mezger, P. G., Gaume, R., \& Johnston, K. J. 1992, A\&A, 256, 640

McBreen, B., Fazio, G. G., Stier, M., \& Wright, E. L. 1979, ApJ, 232, L183

Mead, K. M., Evans, N. J., Il, Kutner, M. L., \& Natta, A. 1989, in Molecular Clouds in the Milky Way and External Galaxies, ed. J. S. Young \& R. L. Snell (Berlin: Springer-Verlag), 271

Mendoza, E. E., \& Gómez, T. 1980, MNRAS, 190, 623

Menten, K. M., \& Batrla, W. 1989, ApJ, 341, 839

Menten, K. M., Serabyn, E., Güsten, R., \& Wilson, T. L. 1987, A\&A, 177, L57

Mezger, P. G., Chini, R., Kreysa, E., Wink, J. E., Salter, C. J. 1988, A\&A, 191, 44

Mezger, P. G., Sievers, A. W., Haslam, C. G. T., Kreysa, E., Lemke, R., Mauersberger, R., \& Wilson, T. L. 1992, A\&A, 256, 631

Mezger, P. G., Wink, J. E., \& Zylka, R. 1990, A\&A, 228, 95

Moore, T. J. T., \& Chandler, C. J. 1989, MNRAS, 241, 19P

Moriarty-Schieven, G. H., Snell, R. L., \& Hughes, V. A. 1989, ApJ, 347, 358

Morris, M. 1975, ApJ, 197, 603

Mundy, L. G., Mangum, J. G., \& Salter, M. J. 1993, in preparation
Mundy, L. G., Wootten, A., \& Wilking, B. A. 1990, ApJ, 352, 159

Nakanaga, T., Kondo, S., \& Saëki, S. 1982, J. Chem. Phys., 76, 3860

Neckel, T. 1978, A\&A, 69, 51

Nerf, R. B. 1975, J. Molec. Spectrosc., 58, 451

Norris, R. P., Booth, R. S., Diamond, P. J., and Porter, N. D. 1982, MNRAS, 201, 191

Person, W. B., \& Zerbi, G., eds. 1982, Vibrational Intensities of Infrared and Raman Spectra (New York: Elsevier)

Persson, S. E., Geballe, T. R., Simon, T., Lonsdale, C. J., \& Baas, F. 1981, ApJ, 251, L85

Phillips, T. G., Knapp, G. R., Huggins, P. J., Werner, M. W., Wannier, P. G., Neugebauer, G., \& Ennis, D. 1981, ApJ, 245, 512

Plambeck, R. L., \& Menten, K. A. 1990, ApJ, 364, 555

Rayner, J., McLean, I., McCaughrean, M., \& Aspin, C. 1989, MNRAS, 241,469

Reid, M. J., \& Ho, P. T. P. 1985, ApJ, 288, L17

Reid, M. J., Myers, P. C., \& Bieging, J. H. 1987, ApJ, 312, 830

Reuter, D. C., Nadler, S., Daunt, S. J., \& Johns, J. W. C. 1989, J. Chem. Phys., 91, 646

Richardson, K. J., Sandell, G., \& Krisciunas, K. 1989a, A\&A, 224, 199

Richardson, K. J., Sandell, G., White, G. J., Duncan, W. D., \& Krisciunas, K. 1989b, A\&A, 221, 95

Rodriguez, L. F., Cantó, J., \& Moran, J. M. 1982, ApJ, 255, 103

Schulz, A., Güsten, R., Zylka, R., \& Serabyn, E. 1991, A\&A, 246, 570

Scoville, N. Z., \& Kwan, J. 1976, ApJ, 206, 718

Snell, R. L., \& Bally, J. 1986, ApJ, 303, 683

Snell, R. L., Scoville, N. Z., Sanders, D. B., \& Erickson, N. R. 1984, ApJ, 284,176

Straw, S. M., \& Hyland, A. R. 1989, ApJ, 340, 318

Straw, S. M., Hyland, A. R., \& McGregor, P. J. 1989, ApJS, 69, 99

Takano, T., Fukui, Y., Ogawa, H., Takaba, H., Kawabe, R., Fujimoto, Y., Sugitani, K., \& Fujimoto, M. 1984, ApJ, 282, L69

Thronson, H. A. 1986, ApJ, 306, 160

Townes, C. H., \& Schawlow, A. L. 1975, Microwave Spectroscopy (New York: Dover)

Turner, B. E. 1992, private communication

Turner, J. L., \& Welch, W. J. 1984, ApJ, 287, L81

Ulich, B. L., \& Haas, R. W. 1976, ApJS, 30, 247

Walker, C. K., Adams, F. C., \& Lada, C. J. 1990, ApJ, 349, 515

Walker, C. K., Lada, C. J., Young, E. T., Maloney, P. R., \& Wilking, B. A. 1986, ApJ, 309, L47

Walker, C. K., Lada, C. J., Young, E. T., \& Margulis, M. 1988, ApJ, 332, 335

Walmsley, C. M., \& Ungerechts, H. 1983, A\&A, 122, 164

Werner, M. W., et al. 1980, ApJ, 242, 601

Whittet, D. C. B. 1974, MNRAS, 168, 371

Wilking, B. A., \& Claussen, M. J. 1987, ApJ, 320, L133

Wilson, T. L., Johnston, K. J., \& Mauersberger, R. 1991, A\&A, 251, 220

Woody, D. P., Scott, S. L., Scoville, N. Z., Mundy, L. G., Sargent, A. I., Padin, S., Tinney, C. G., \& Wilson, C. D. 1989, ApJ, 337, L41

Wootten, A. 1989, ApJ, 337, 858

Wootten, A., Loren, R. B., Sandqvist, A., Friberg, P., \& Hjalmarson, Å. 1984, ApJ, 279, 633

Wootten, A., Snell, R. L., \& Evans, N. J., II. 1980, ApJ, 240, 532

Wright, M. C. H., Dickel, H. R., \& Ho, P. T. P. 1984, ApJ, 281, L71

Wynn-Williams, C. G., Becklin, E. E., \& Neugebauer, G. 1972, MNRAS, 160,1

Zhou, S., Evans, N. J., II, Güsten, R., Mundy, L. G., \& Kutner, M. L. 1991, ApJ, 372, 518

Zhou, S., Evans, N. J., II, \& Mundy, L. G. 1990, ApJ, 355, 159 